RESPONSE OF MARTIAN GROUND ICE TO ORBIT-INDUCED CLIMATE CHANGE

By

Matthew Allyn Chamberlain

\author{
A Dissertation Submitted to the Faculty of the \\ DEPARTMENT OF PLANETARY SCIENCES \\ In Partial Fulfillment of the Requirements \\ For the Degree of \\ DOCTOR OF PHILOSOPHY \\ In the Graduate College \\ THE UNIVERSITY OF ARIZONA
}




\section{THE UNIVERSITY OF ARIZONA GRADUATE COLLEGE}

As members of the Dissertation Committee, we certify that we have read the dissertation prepared by Matthew Allyn Chamberlain entitled Response of Martian Ground Ice to Orbit-Induced Climate Change and Other Topics

and recommend that it be accepted as fulfilling the dissertation requirement for the Degree of Doctor of Philosophy

Date: December 13, 2005

William V. Boynton

Date: December 13, 2005

Robert H. Brown

Date: December 13, 2005

Adam P. Showman

Date: December 13, 2005

Renu Malhotra

Date: December 13, 2005

Rojer V. Yelle

Final approval and acceptance of this dissertation is contingent upon the candidate's submission of the final copies of the dissertation to the Graduate College.

I hereby certify that I have read this dissertation prepared under my direction and recommend that it be accepted as fulfilling the dissertation requirement.

Dissertation Director: William V. Boynton

Date: December 13, 2005 


\section{STATEMENT BY AUTHOR}

This dissertation has been submitted in partial fulfillment of requirements for an advanced degree at the University of Arizona and deposited in the University Library to be made available to borrowers under rules of the Library.

Brief quotations from this dissertation are allowable without special permission, provided that accurate acknowledgment of source made. Requests for permission for extended quotation from or reproduction of this manuscript in whole or in part may be granted by the head of the major department or the Dean of the Graduate College when in his or her judgment the proposed use of the material is in the interests of scholarship. In all other instances, however, permission must be obtained from the author.

SIGNED:

M. A. Chamberlain 


\section{ACKNOWLEDGEMENTS}

First I thank those who made this whole dissertation possible. Thanks to LPL as a department for admitting an aussie alien into the best planetary science program there is. I had great supervisors in my LPL years; Bob Brown and Bill Boynton who gave me the opportunity to extend myself and try some challenging projects, and demonstrated what life can be like at the sharp end of planetary exploration.

I would like to thank those who have helped in various technical aspects of the work shown in this dissertation. I thank Bob Haberle for sharing radiative transfer subroutines from the AMES GCM that helped in the development of my own models. I am also grateful to all those who have processed and provided such great datasets of the properties of the surface of Mars that are available to everyone to use: Phil Christensen and the TES team for the albedo, Michael Mellon and Nathaniel Putzig and the TES team again for the thermal inertia, Michael Smith and the TES team once more for water column abundances, and David Smith and the MOLA team for elevation. I also appreciate the useful discussions within the GRS team while I have worked on these projects. Big thanks also to Mike Finch who arrived to the GRS group with his mapmaking software just the right time and saved me weeks of working it out myself. Thanks to Maria Schuchardt for assistance looking through the Viking images. I thank Josh Emery and Dale Cruikshank for comments while writing the Himalia chapter, and Rick McCloskey for assistance processing the VIMS data. And thanks to Josh and Carl Hergenrother for letting me tag along on various observing trips up the mountains over the years.

Next I must thank those who have helped my work in indirect ways, but ways that were just as important on this journey.

Thanks to officemates, Curtis Cooper and Mike Bland who were always able to discuss the issues, science and sundry. Also to Moses Milazzo who was more than able to relate to the challenges of juggling a young family and $\mathrm{PhD}$ degree, and the need to play a hand of cards. Thanks also to rest of the grad student population for encouragement and entertainment throughout my years at LPL.

Thanks to the Santa Catalinas, who may have always been a distraction staring at me through the window, but also a great stress relief at the appropriate times.

A big thank you to my family for hanging in the there and keeping me sane. And thanks to my parents for instilling in me the right attitude to work and life to get through all these challenges. 


\section{TABLE OF CONTENTS}

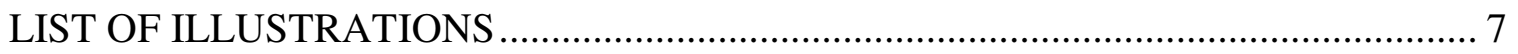

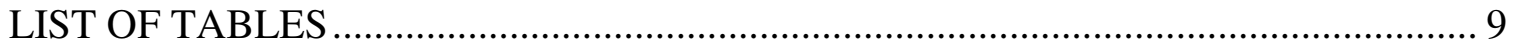

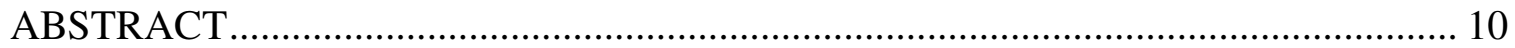

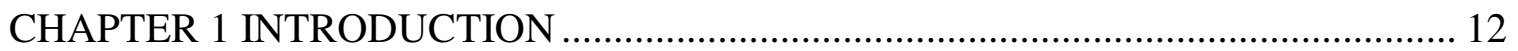

CHAPTER 2 RESPONSE OF MARTIAN GROUND ICE TO ORBIT-INDUCED

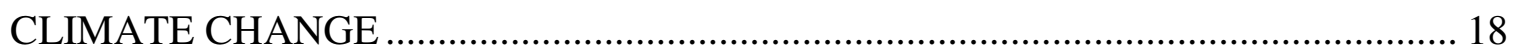

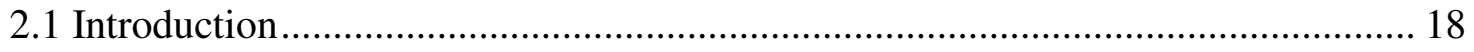

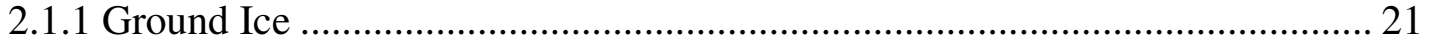

2.1.2 Martian Water Cycle .................................................................................. 27

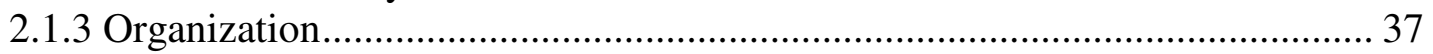

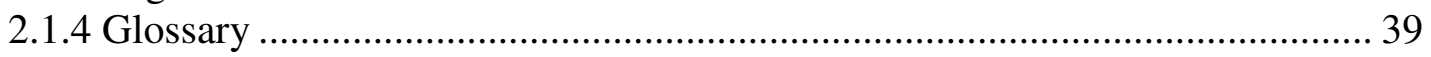

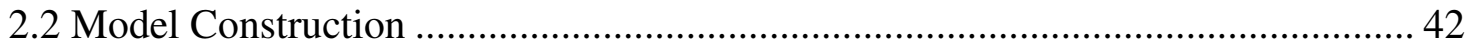



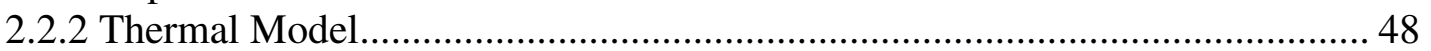



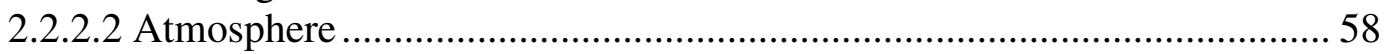

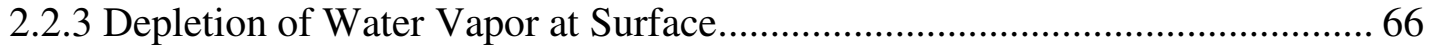

2.2.3.1 Variation in Water Content of Atmospheric Column with Seasons ......... 70

2.2.3.2 Diurnal Depletion of Water Vapor in the Near-Surface ........................... 75

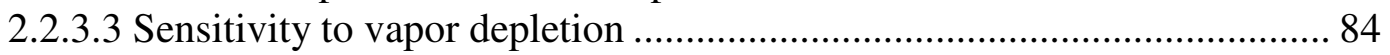

2.2.4 Determining Stable Depth of Ice ................................................................. 88

2.2.4.1 Distribution of Atmospheric Water ........................................................ 95

2.2.4.2 Generating Maps of Ground Ice Distribution ............................................ 96

2.2.5 Climate at Other Epochs ........................................................................... 102



2.2.5.2 Water Vapor at Other Epochs ………................................................. 106





2.3.1.1 Discussion of model components ............................................................ 119

2.3.1.2 Response of ground ice to changes in atmospheric water content.......... 123

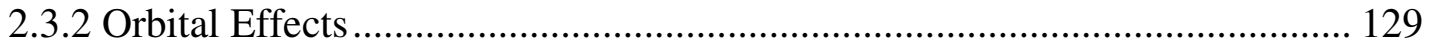

2.3.2.1 Effect of Position of Perihelion......................................................... 129

2.3.2.2 Effect of Obliquity ........................................................................... 135

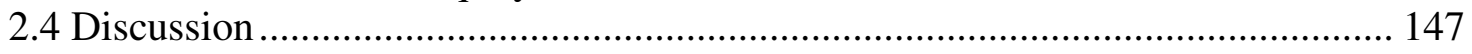






\section{TABLE OF CONTENTS - Continued}

CHAPTER 3 NEAR-INFRARED SPECTROSCOPY OF HIMALIA ……………….... 162

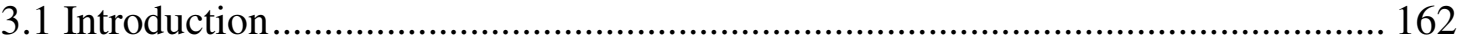







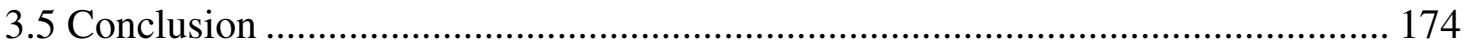

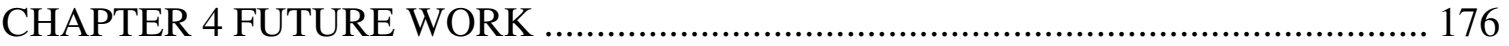



4.2 Vapor Diffusion ...................................................................................... 179

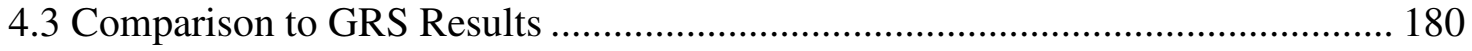



APPENDIX A: THERMAL MODEL APPLIED TO CORRECT THE HIMALIA

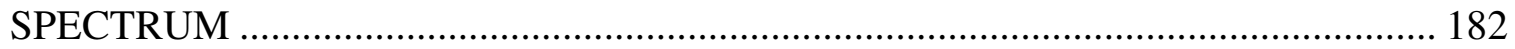

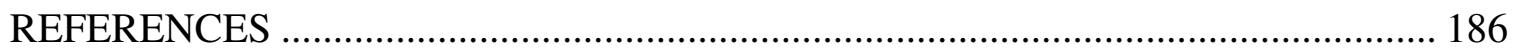




\section{LIST OF ILLUSTRATIONS}

Figure 1 Recent history of martian obliquity. Figure based on Ward (1992).................. 20

Figure 2 Water equivalent content of the surface of Mars based on observed hydrogen gamma ray flux. 23

Figure 3 The variation in ground ice extent as a function of obliquity as predicted by

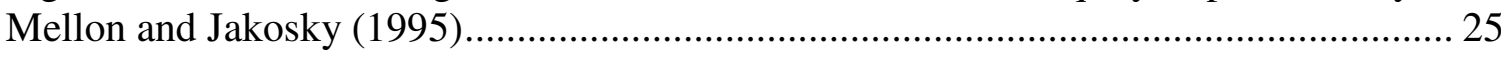

Figure 4 Two models showing extent of martian ground ice for the current epoch......... 26

Figure 5 The variation of the zonal water vapor column observed by TES over 2 martian years 30

Figure 6 Annual average water column observed over the surface of Mars .................... 30

Figure 7 Water cycle results from a GCM at an obliquity of $45^{\circ}$................................... 37

Figure 8 Depth to stable ice estimated with a variety of models ...................................... 47

Figure 9 Sample output from thermal model. …………………………………….. 53

Figure 10 Sunrise and sunset times as a function of $L_{s}$ for a location at $70^{\circ} \mathrm{N}$............... 53

Figure 11 Sample seasonal temperature profile .......................................................... 54

Figure 12 The changes in annual average surface temperatures, with and without the

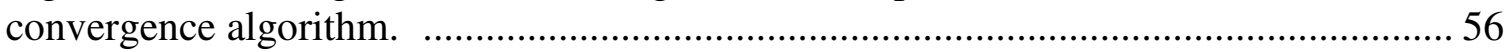

Figure 13 Average temperatures over latitudes with different atmospheric models ........ 64 Figure 14 Cartoon showing the process of vapor depletion of the near-surface atmosphere.

Figure 15 Estimated atmospheric water cycle over a dark, rocky surface at various

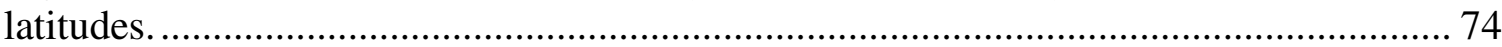

Figure 16 Water in the atmosphere over dark, rocky ground at $40^{\circ} \mathrm{N}$........................... 79

Figure 17 Vapor depletion within the diurnal cycle ....................................................... 79

Figure 18 The ratio of the average water vapor density of the near-surface atmosphere. 82

Figure 25 Change in water cycle for a model at $40 \mathrm{~N}$ and dark, rocky surface................ 85

Figure 26 Effective ratios of annual average vapor density over dark, rocky surface as

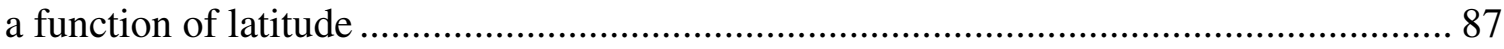

Figure 19 Variations in surface model results as a function of ice depth........................ 91

Figure 20 Determining depth to stable ice. ................................................................ 94

Figure 21 Martian thermal inertia map. .................................................................. 98



Figure 23 Difference in model results due to interpolation from the look-up table. .... 101 Figure 24 Water carrying capacity of the atmosphere over the north pole in the

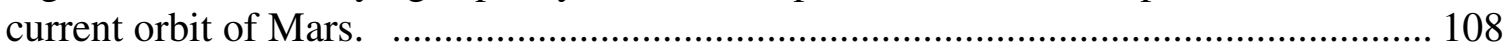

Figure 27 Best estimate of depth to stable ground ice on Mars ...................................... 112 Figure 28 The distribution of martian ground ice for the present epoch using the same

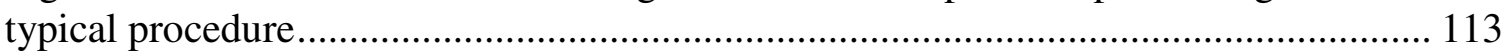

Figure 29 The average surface temperature of Mars in the present epoch, ................... 115 


\section{LIST OF ILLUSTRATIONS - Continued}

Figure 30 The effect of ground ice on model results at high latitudes as a function of thermal inertia.

Figure 31 Variations in the distribution of ground ice as a function of the water content of the atmosphere.

Figure 32 Atmospheric water carrying capacity

Figure 33 Distribution of ground ice if vapor depletion near the surface is not taken into account... 128

Figure 34 Atmospheric water carrying capacity for different perihelion- $\mathrm{L}_{\mathrm{s}}$.................. 130



Figure $36 \mathrm{CO}_{2}$ as a function of obliquity, as used for this project................................. 136

Figure 37 Base atmospheric water content as a function of obliquity. ......................... 138

Figure 38 Atmospheric water-carrying capacity over exposed surface ice as a

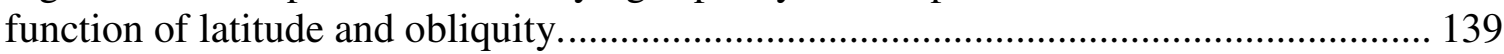

Figure 39 The distribution of ground ice for different obliquities.................................. 141

Figure 40 Various styles of dissected mantle mapped.................................................. 149

Figure 41 Distribution of various dissected mantle textures. ........................................ 150

Figure 42 Histogram of the latitudinal distribution dissected mantles........................... 153

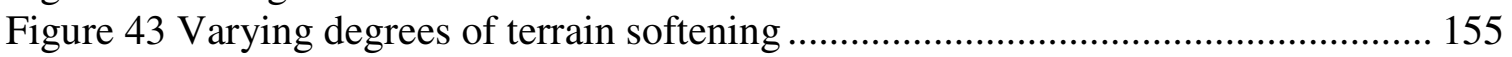

Figure 44 Normalized reflectance spectrum of Himalia................................................ 168

Figure 45 The convolved reflectance spectrum with model spectra............................... 172

Figure 46 Thermal radiation calculated from "Thermod" .............................................. 185 


\section{LIST OF TABLES}

Table 1 Summary of parameter values for thermal model and determining ice depth..... 52

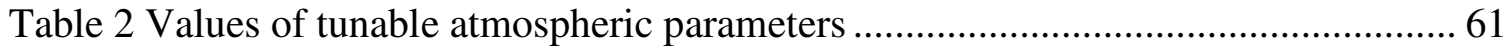

Table 3 Sample results of the water vapor depletion algorithm. ................................... 81

Table 4 Summary of ground-ice distribution for different models.............................. 110

Table 5 Summary of ground-ice distribution for different models at high obliquity ..... 143

Table 6 Observation parameters for Himalia.......................................................... 164 


\begin{abstract}
A thermal model is developed to find the distribution of stable near-surface ground ice on Mars that is in diffusive contact with the atmosphere for past and present epochs. Variations in the orbit of Mars are able to drive climate changes that affect both surface temperatures and atmospheric water content so the distribution of ground ice will vary significantly in past epochs. A technique is developed to correct the average water vapor density above the surface for depletion due to diurnal frost formation. Also presented is a simple model to estimate the atmospheric water content, based on the water vapor carrying capacity of the atmosphere over water ice on the martian surface.

Maps of the distribution of ground ice are generated for the present epoch of Mars with varying amounts of water vapor in the atmosphere. The water vapor depletion scheme restricts the extent of stable ground ice as more water is put into the atmosphere so that ice never becomes stable at low latitudes. As the position of the perihelion varies, the extent of ground ice changes several degrees in the latitudinal extent, primarily in the northern hemisphere. The extent of ground ice is sensitive to the obliquity of Mars, however high obliquities are still not able to make ground ice stable at low latitudes. Finding ice is never stable at low latitudes is consistent with the lack of terrain softening at low latitudes and models that indicate Mars had high obliquities for much of its history.
\end{abstract}


Also presented is the first L-band spectrum of an irregular satellite from the outer Solar System. Spectra of Himalia were obtained with the Visual and Infrared Mapping Spectrometer onboard the Cassini spacecraft. The Himalia spectrum is essentially featureless, showing a slight red slope and a suggestion of an absorption feature at $3 \mu \mathrm{m}$ that would indicate the presence of water. Better measurements of the spectrum of Himalia, particularly in the region of the apparent $3-\mu \mathrm{m}$ band, could help determine whether water is present, and if so, in what form. 


\section{CHAPTER 1}

\section{INTRODUCTION}

The chapters that follow represent the most finished products from my 5 or so years of research at Lunar and Planetary Laboratory, Tucson. The chapter that makes up the largest part of this thesis, involves the use of near-surface thermal models to predict the distribution of ground ice on Mars. Much of my time here has been spent developing and refining these models so that they reproduce the ground-ice distribution that has been observed and published by others for the present epoch. I then applied the models to determine the extent of martian ground ice in past epochs and relate this to the distribution of ice-related landforms. There are a number of other promising projects that follow on from this work that also use models I developed. The other chapter I have included shows the first L-band spectrum of an irregular satellite of any jovian planet. I present a spectrum of Himalia, a satellite of Jupiter which shows evidence of water in some form.

If there is a single theme that ties everything together I would call it "Water in the Middle Solar System." In one chapter I discuss ice near the surface of Mars at the outside of the inner Solar System and in another I detect water on the inside of the outer Solar System. Water is of course a popular substance to find in the Solar System. Much effort is going into the "Follow the Water" strategy currently driving much of the present 
planetary exploration. Not least of which is the coming Phoenix mission that will land in the polar regions of Mars and study water there in all its phases. Interest in water is largely motivated by the search for life, past or present, and the presumed vital role that water plays in supporting life. For my own work I focus on the physical effects of water ice (or other phases) on its environment and climate; I do not speculate about potential habitats.

Another hot topic I get to touch on in the main chapter of my dissertation is climate change. Mars presents itself as a climate-change laboratory, if only we could decipher what the planet was telling us. Climate change on Mars is likely to be much more extreme than what is experienced on Earth. On one hand, the climate of Mars is simpler than on Earth; the atmosphere is much thinner, there are no oceans, the latent heat of water does not play an important role and living things do not grow all over the surface. On the other hand, Mars has no large natural satellite and the obliquity of the planet is less stable. The wide range of obliquity that Mars has experienced modifies the distribution of insolation over the latitudes, which is a strong driver of climate. There seems to be a strong relation between relatively small variations in the obliquity and orbit of Earth and cyclical climate change with periods of 10 s to 100 s thousands of years.

Studying water on Mars is tough. There is a lot work that has been done already (some good, some not-so-good), and there are a lot of people still studying the planet. Not just is there a lot of competition, but there are some big names who have done a lot of work on Mars. One must be somewhat careful if they are going to claim that Bruce 
Jakosky or some other Mars science deity is wrong. I prefer instead to think that I am modifying our understanding of Mars.

I first started developing my near-surface thermal models for Mars as I was preparing for my orals. At the time I had a one-layer thermal model, where 'one-layer' is used to imply that thermal properties were assumed to be uniform with depth (the model did include more than one depth layer). During my orals the suggestion was made to enable my thermal models to include the change in the thermal properties where ice is present in the ground, i.e. create a two layer model. This process by itself would have been worth publishing at the time, however a paper that included just this process appeared soon afterward (Mellon et al. 2004) - the problem with studying a popular topic. Not to be discouraged, I worked on and developed my own version of the two layer thermal model and applied it to past epochs of Mars which has still not been done with two-layer thermal models. This is the main thrust of the work presented in Chapter 2. I also included some new components to the model and explain some relevant physical processes. At the end of this chapter (Section 2.4), I include some images of the surface of Mars and discuss the distribution of some ice-related features such as terrain softening and dissected mantles. These features support my conclusion that ground ice should not be globally stable in past epochs. Using some actual images appeal to the geologist and the experimental scientist who is still within.

This chapter is still about to be submitted for publication (hopefully to not be delayed with more dissertation corrections - I really do not want to be scooped again). The version that will be submitted will be somewhat condensed, particularly the 
introduction and model description sections. I have taken advantage of the dissertation format to include extra figures to aid the reader. This chapter is written in the first person plural for publication, though the work that is shown is primarily my own. Bill Boynton and I have had useful discussions along the way and he has given me many helpful suggestions and kept tabs on my progress. The models and the solutions presented are the result of beating my own head against the office walls. All the programs I use I have developed myself from scratch. The only exception is a set of Ames Mars GCM subroutines I obtained (thanks to Bob Haberle of NASA Ames Research Center) to calculate radiative transfer through the atmosphere. However, I did not regularly use these subroutines in my thermal models used to generate maps of stable ground ice. Instead I used them to validate subroutines of atmospheric radiation that I developed myself and thatran a lotquicker.

I have been able to use the same thermal model to estimate ice depth by fitting models to observed surface temperatures. I used my twe layer thermal model to explain anomalies that appear in the thermal inertia maps by Putzig et al. (2005). I was able to compare my twe layer model results direct to TES surface temperature data (thanks to Tim Titus of the USGS) and found a better fit to the data. In the process of fitting I derived my own thermal inertia and estimated the depth to ice as an added bonus. I showed preliminary results of this work in a Lunar and Planetary Science Conference poster (Chamberlain and Boynton 2005) and it is described in this thesis under future work. This is just one example of other processes and surface properties that could be revealed by fitting detailed thermal models to observed surface temperatures. With a little 
more work, results from this project fitting TES surface temperatures to ice depth and other properties will be ready for a peer-reviewed publication as well.

Chapter 3 contains a paper from an infrared spectroscopy paper that I wrote a couple of years ago (Chamberlain and Brown 2004). This paper is the result of a project that involved the extraction, data processing and interpretation of spectroscopic data from the Visual and Infrared Spectrometer (VIMS) onboard the Cassini spacecraft. VIMS took observations of Himalia, an irregular jovian satellite, as Cassini flew past Jupiter at the end of 2000 on its way to Saturn. For this work I had help from Bob Brown who made suggestions and provided calibration data. Codes that used the calibrations and processed the data were my own.

The crux of this project was squeezing the noisy data to: 1) find the object, and 2) obtain a spectrum. Not even including the cosmic radiation, the Himalia signal-to-noise was $\sim 1$ in any single frame and the pointing of Cassini at the time was poorly controlled; Himalia was not even in the field of view in most of the frames taken. I manually went through all the frames and hand picked the pixels that contained the signal from Himalia. Cosmic ray hits on the detector array would knock out several wavelength channels from every pixel. I developed schemes to completely remove effects of these spikes from the spectrum of Himalia.

With the processed spectrum of Himalia I was able identify a shallow absorption feature at $3 \mu \mathrm{m}$ corresponding water band which could be water ice or hydrated minerals. Thermal corrections to the long-wavelength channels of the spectrum $(\sim 5 \mu \mathrm{m})$ used 
thermal results from a model by Bob Brown that is written up in Appendix A. I did this spectroscopy project before I developed my own thermal models for Mars. 
CHAPTER 2

\title{
RESPONSE OF MARTIAN GROUND ICE TO ORBIT-INDUCED CLIMATE
}

\author{
CHANGE
}

\subsection{Introduction}

The distribution of near-surface ground ice on Mars is intimately associated with the martian climate. This near-surface ice is able to interact with the atmosphere on short geological timescales and is distinct from the ice deeper in the cryosphere of Mars. The extent of stable ground ice at any epoch is related to the average temperatures of the surface and the content of water in the atmosphere, both of which are determined by the climate of that epoch. Just based on theories of orbit evolution, martian climate should have changed significantly even in recent geological history. Ground ice plays a significant role in various landforms identified in images of the martian surface. Understanding the distribution of ground ice both now and in Mars' past help in understanding where ice-related landforms should be found, if our understanding is correct. Ice stability does not necessarily restrict where ice may be found at any 
particular time. Water ice at a location may be residual from a previous epoch or other processes such as circulation may be supplying water more rapidly than it is subliming.

In the case of the Earth, climate cycles over the last 10 s and 100 s of thousands of years, as represented in various stratigraphic records, appear to be closely associated with minor variations in the Earth's orbit (Milankovitch 1941; Hinnov 2000). Though Earth and Mars have similar angles of obliquityat present, $23.45^{\circ}$ and $25.19^{\circ}$ respectively, Mars does not have a large stabilizing satellite like Earth. Models of martian obliquity evolution show a much wider range of variation in orbit parameters which presumably would be capable of driving climate change more extreme than experienced on Earth (e.g., Ward 1992). The primary driver of climate change is the angle of obliquity which modifies the distribution of energy from the Sun, insolation, over the latitudes. Over the last few million years, the obliquity of Mars has swung from $15^{\circ}$ to $35^{\circ}$; going back several million years the obliquity was as high as $45^{\circ}$. Figure 1 shows how the obliquity of Mars has varied over the last few million years. While the long-term history of the orbit and obliquity of Mars is not known exactly, a statistical analysis shows that the average obliquity of Mars over the last few billion years is $\sim 38^{\circ}$ (Laskar et al. 2004), much higher than the present obliquity. Eccentricity and the position of perihelion also modify the insolation and climate to a smaller degree. Mars is also particularly susceptible to climate change because surface temperatures are capable of dropping below the condensation point of the main component of the atmosphere, $\mathrm{CO}_{2}$. At present, of the order of $25 \%$ of the atmosphere condenses out onto the winter pole as $\mathrm{CO}_{2}$ ice creating a large seasonal variation in the atmospheric pressure. At higher obliquities 
these seasonal variations will become more extreme and seasonal polar caps will be more extensive. At lower obliquities, colder temperatures at the poles may form large caps of permanent $\mathrm{CO}_{2}$, lowering the average global surface pressure. Changes in climate and surface temperatures will also modify the water cycle and water content of the atmosphere; these changes will be discussed later in this section.

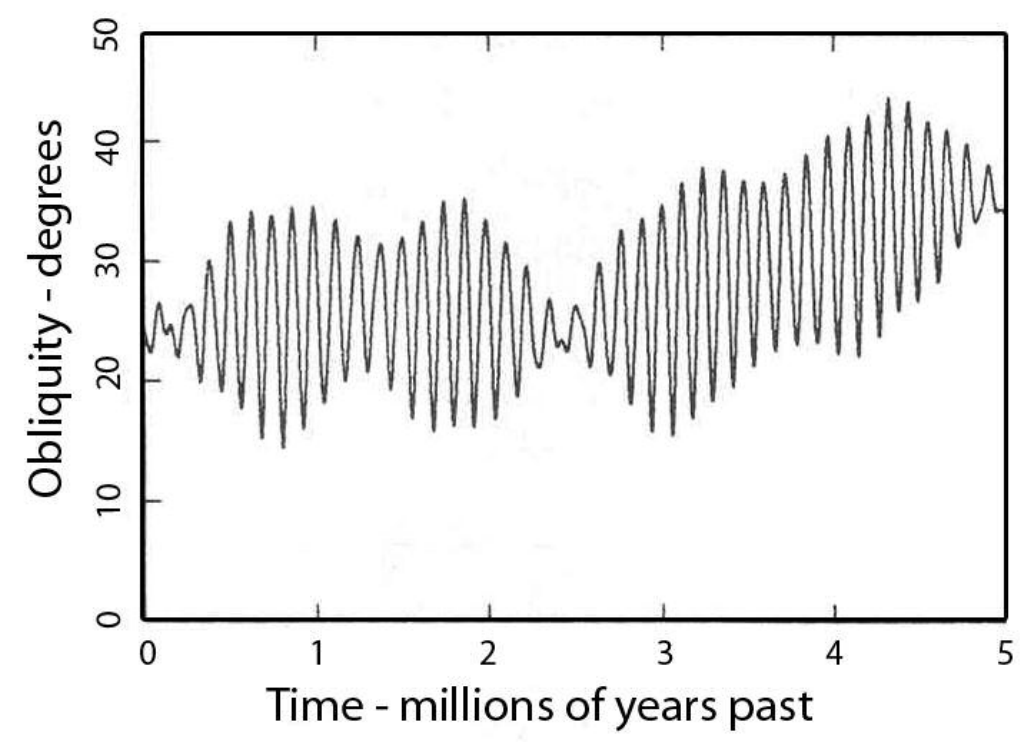

Figure 1 Recent history of martian obliquity. Figure based on Ward (1992).

On Earth, there are many unique landforms that are only associated with the presence of ground ice or permafrost - for example frost-heaving, polygons and patterned ground (Black 1976). While many terrestrial cold-climate features are associated with the seasonal freeze-thaw cycle which may not be applicable to Mars (which never is warm enough to thaw), there are still many observed martian landforms that suggest the 
action of ground ice (e.g., Squyres and Carr 1986; Carr 1996). These landforms include: lobate debris aprons, terrain softening, rampart craters, polygonal fractures, patterned ground, and thermokarst. There are also features suggesting glacial activity such as eskers and moraines, and of volcano-ice interactions.

Recent images at high resolution of the martian surface have revealed mantle covers showing varying degrees of dissection and viscous flow on the martian surface and have been mapped out by Mustard et al. (2001) and Milliken and Mustard (2003). It has been assumed that the 'dissected' nature of these mantles is due to volume loss by the sublimation of entrained water ice. The extent of these dissected mantles has been attributed to the extent of deposition of ice from the atmosphere during periods of high obliquity and later desiccation at low obliquities.

\subsubsection{Ground Ice}

The presence of ground ice on Mars has long ben suspected based on surface temperatures and atmospheric water content (Leighton and Murray 1966). Hydrogen gamma ray and neutron fluxes observed by the Gamma Ray Spectrometer (GRS) onboard the Mars Odyssey spacecraft have detected high hydrogen contents over high-latitude regions of Mars (Feldman et al. 2002; Boynton et al. 2002; Feldman et al. 2004b; Boynton et al. 2006), see Figure 2. Surface thermal models can predict the extent of ground ice that is stable to vapor diffusion with the atmosphere, for example Mellon and Jakosky (1993), Mellon and Jakosky (1995), Mellon et al. (2004), and Schorghofer and 
Aharonson (2005). Thermal models assume there is diffusive contact between the atmosphere and subsurface so that the atmosphere can emplace or deplete water if the ice is stable or not. Other processes may form ice deposits either at or below the surface, by either ice migration within the ground or precipitation from the atmosphere. The thermal model results based on vapor diffusion are still useful in determining where ice deposited by these other mechanisms would be stable to sublimation. Indeed, the high water-ice equivalent hydrogen content detected by the GRS (Feldman et al. 2004b; Boynton et al. 2006) across the high latitudes of Mars suggest that there is too much ice present to be explained by filling pore spaces of a regolith by vapor diffusion. 


\section{Data from 2001 Mars Odyssey Gamma Ray Spectrometer

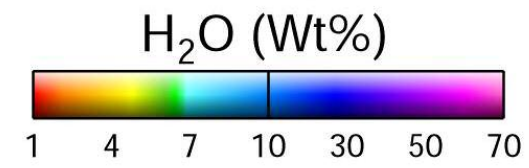

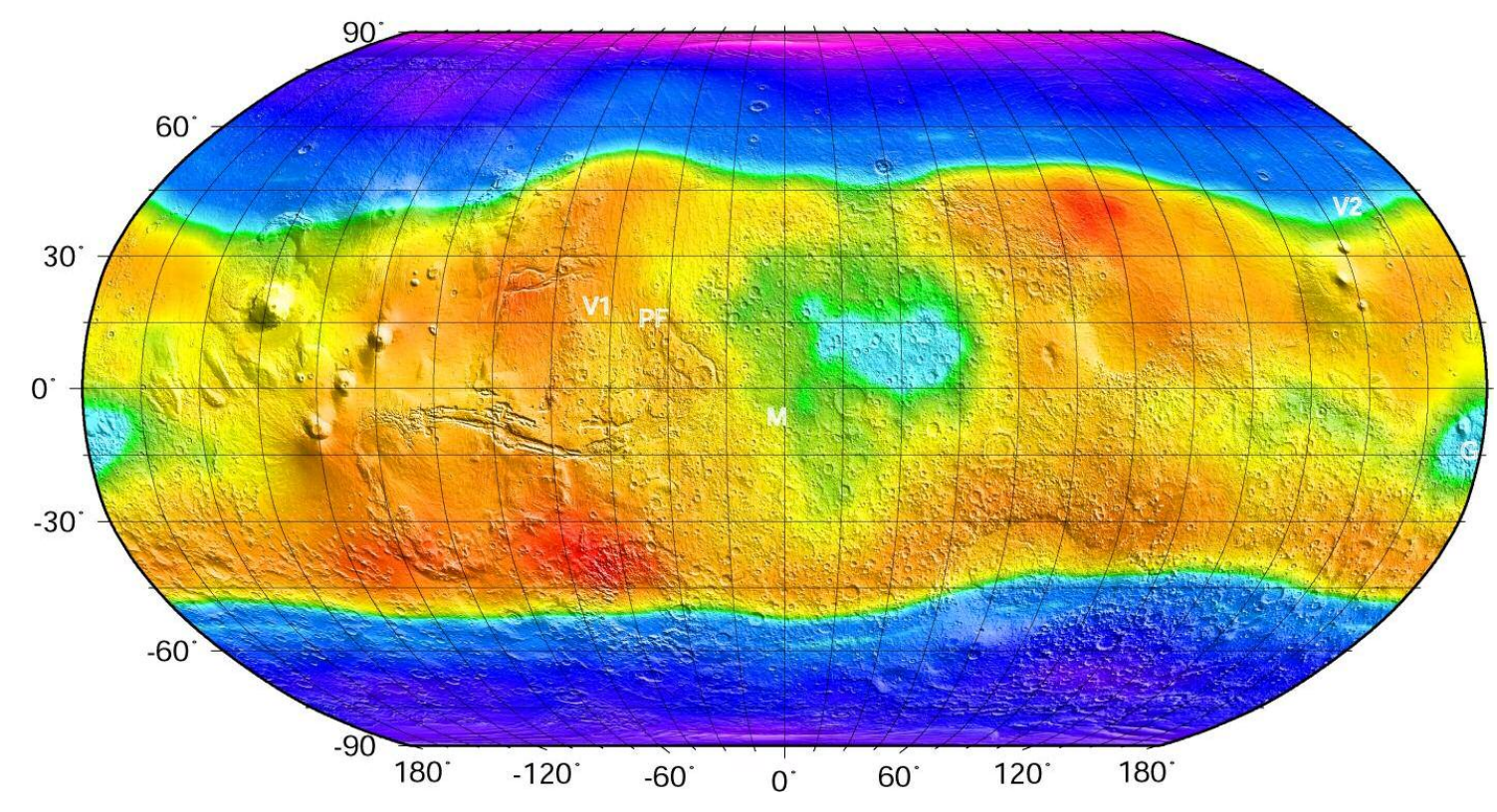

Figure 2 Water equivalent content of the surface of Mars based on observed hydrogen gamma ray flux. The color scale has been skewed so that features at both high and low latitudes are visible. Water equivalent contents that imply the presence of ground ice appear as deep blue to purple. Water contents shown over low latitudes are low and may be explained by the presence of hydrated minerals and/or salts. This figure is based on Boynton et al. (2006). 
A one-layer near-surface thermal model was used by Mellon and Jakosky (1993) and Mellon and Jakosky (1995) to determine the distribution of ground ice on Mars; the 1993 paper determined the distribution for Mars in the present epoch and the 1995 paper explored past distributions at different obliquities. The 1995 paper indicated that ground ice would become globally stable at obliquities as low as $32^{\circ}$, as shown in Figure 3 . The model was one-layer in the sense that it assumed the thermal properties of the ground were uniform with depth, or at least within the top few seasonal skin depths. Mellon et al. (2004) recalculated the distribution of ground ice for Mars in the present epoch using a two-layer near-surface thermal model that allowed for a change in the thermal properties in the ground at a depth where ground ice was present. Including the high thermal inertia properties within the ice-rich layer was found to make ice stable closer to the surface by about a factor of several; this process had been demonstrated previously by Paige (1992). The difference in deptkan be seen by comparing the twqanels in Figure 4 which show the distribution of stable ground ice in the present epoch for one- and twe layer models. Comparisons with the neutron spectrometer results (Feldman et al. 2002) suggest that a globally averaged atmosphere content of 10 to 20 precipitable microns of water (pr- $\mu \mathrm{m})$ best explains the present extent of ground ice (Mellon et al. 2004). Schorghofer and Aharonson (2005) calculate a similar distribution of ground ice on the present Mars using a two-layer thermal model that includes the diffusion of water vapor. They also discuss the water vapor exchange with the atmosphere, extent of seasonally stable frost and sensitivities to changes in albedo and slopes. The extent of ground ice of all these models 
show good qualitative agreement with the GRS observation, indicating that the processes determining ice stability for the present epoch are understood.

Obliquity $=29.3$ degrees

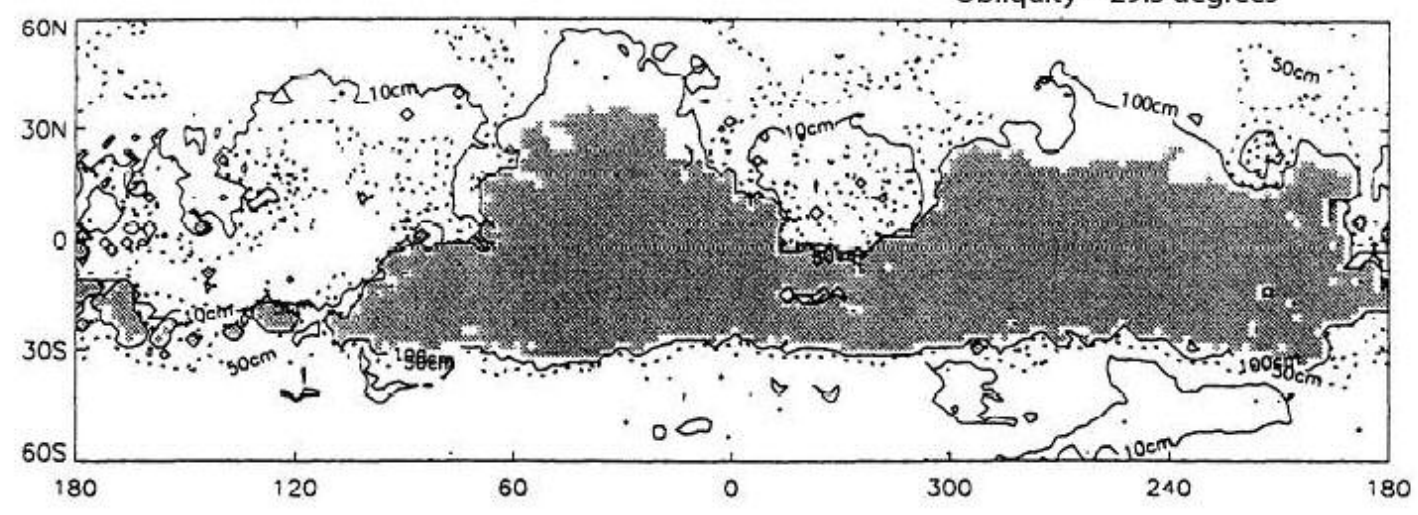

Obliquity $=32.35$ degrees

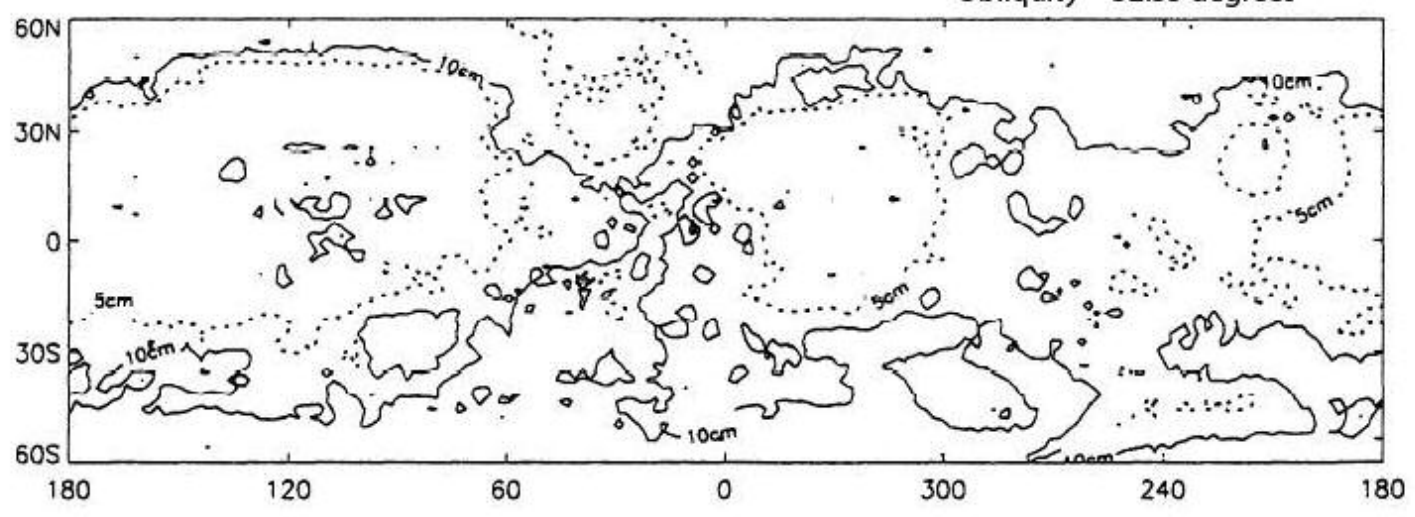

Figure 3 The variation in ground ice extent as a function of obliquity as predicted by Mellon and Jakosky (1995). Contours show depth to stable ice in $\mathrm{cm}(5,10,50$ and 100); shaded regions indicate where ground ice is unstable. 
a)

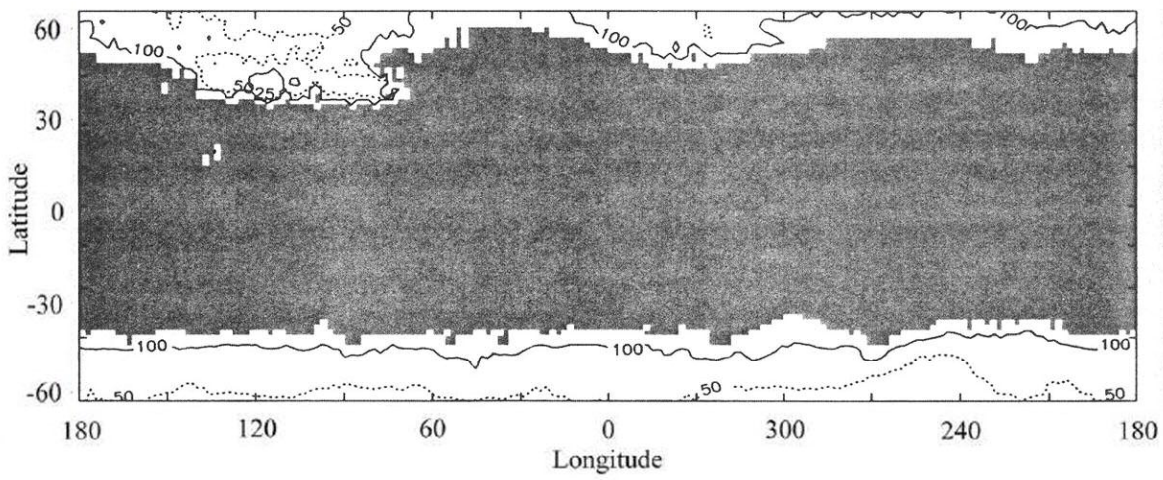

b)



Figure 4 Two models showing extent of martian ground ice for the current epoch. The top panel (a) is based on Mellon and Jakosky (1993) which assumes thermal properties are uniform with depth (contours show depth to stable ice and shaded regions indicate where ice is unstable). The lower panel (b) from Mellon et al. (2004) includes the effect of high thermal inertia within the ice-rich layer - a two-layer model (color is used to indicate stable ice depth and clear regions indicate where ice is unstable). 
The near-surface is only a small part of the martian cryosphere. While it has been demonstrated that near-surface ice is unstable at low latitudes, the subsurface is still well below freezing at depth at these locations. Fanale et al. (1986) estimated the depth to which ground ice might retreat over the history of Mars at all latitudes. They also predict that ice would be stable near the surface poleward of the mid latitudes. At the equator they estimate ice will retreat to a depth of a few hundred to several hundred meters over the history of the planet.

In this work about martian ground ice, we use a two-layer thermal model to determine a ground ice extent in past epochs. The relation between our work and Mellon et al. (2004) is similar to the relation between Mellon and Jakosky (1995) and Mellon and Jakosky (1993). We consider changes in both the position of perihelion and the obliquity angle. We use a new scheme to estimate the atmosphere water content in response to these changes in orbit which is substantially lower than water contents used in Mellon and Jakosky (1995), but is in agreement with recent general circulation model results (Mischna et al. 2003; Mischna and Richardson 2005).

\subsubsection{Martian Water Cycle}

While there had been tentative, if not fanciful, observations of martian canals with flowing water in the late nineteenth century, Mariner 4 in 1965 quickly revealed the cold, dry Mars that we think of today (see Kieffer et al. 1992 for a review of early martian 
observations). The martian water cycle is naturally driven by the climate and forms a crucial part in determining the stability of ground ice. A complete understanding of the water cycle, past or present, would require an understanding of the climate at all times as well, which is well beyond the scope of this work. However, we are able to make some simple assumptions and check that they are reasonable compared to observations and most recent models of the water cycle.

While it is true to think of Mars as dry in an absolute sense, it is also wet in a relative sense. The cold martian temperatures imply that much of the atmosphere is actually close to saturation. Frost has been observed on the martian ground, both around the Viking 2 Lander and the Mars Exploration Rovers indicating there has been enough water in the atmosphere for the ground to condense frost, both on a seasonal and diurnal cycle. At the Viking 2 Lander site water frost formed midway through the autumn season and stayed on the surface until around the end of winter (Svitek and Murray 1990). Water frost has also been seen on the Mars Exploration Rovers early in the morning while the northern hemisphere is in summer and more water is present in the atmosphere (NASA press release 2004).

The present water content of the martian atmosphere is based on observations over several decades. Telescopes on Earth have estimated the water column as far back as the 1960's (Spinrad et al. 1963). The first detailed observations of the global martian water cycle, how water is transported and distributed between the atmosphere and other reservoirs through the seasons, came from the Mars Atmospheric Water Detector (MAWD) onboard the Viking Orbiters (Jakosky and Farmer 1982). More recently, the 
Thermal Emission Spectrometer (TES) (Christensen et al. 2001) onboard the Mars Global Surveyor (MGS) has continued observing atmospheric water abundance. Both datasets show the same general pattern in observed water cycle; highest water column abundances are found in the north polar region during the northern summer, abundances over low latitudes show smaller variations over the season, and water column over in the south shows a smaller peak in the southern summer, if at all (see Figure 5). Over the period of time of ground- and space-based observations, the amount of water in the martian atmosphere has been relatively constant, 10 to $20 \mathrm{pr}-\mu \mathrm{m}$ (precipitable microns of water) has been a typical content. The single disadvantage of the TES dataset is the sunsynchronous orbit and the inability to test for diurnal variation in the water vapor. TES observations do indicate interannual variability in the southern summer. The southern summer in 2001 appeared to have less atmospheric water vapor than the 1999 season. In the 1960's an anomalously high water content was observed from the ground in the southern hemisphere of Mars (Barker et al. 1970). 


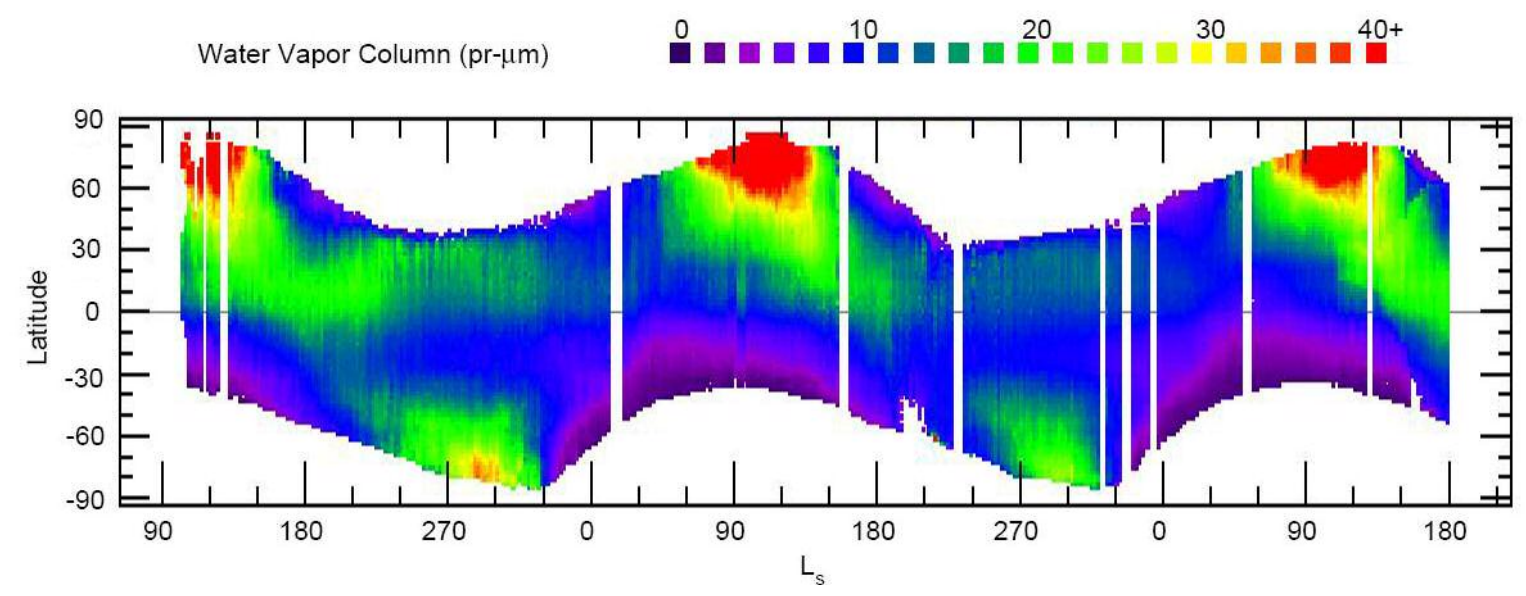

Figure 5 The variation of the zonal water vapor column observed by TES over 2 martian years (Smith 2004).
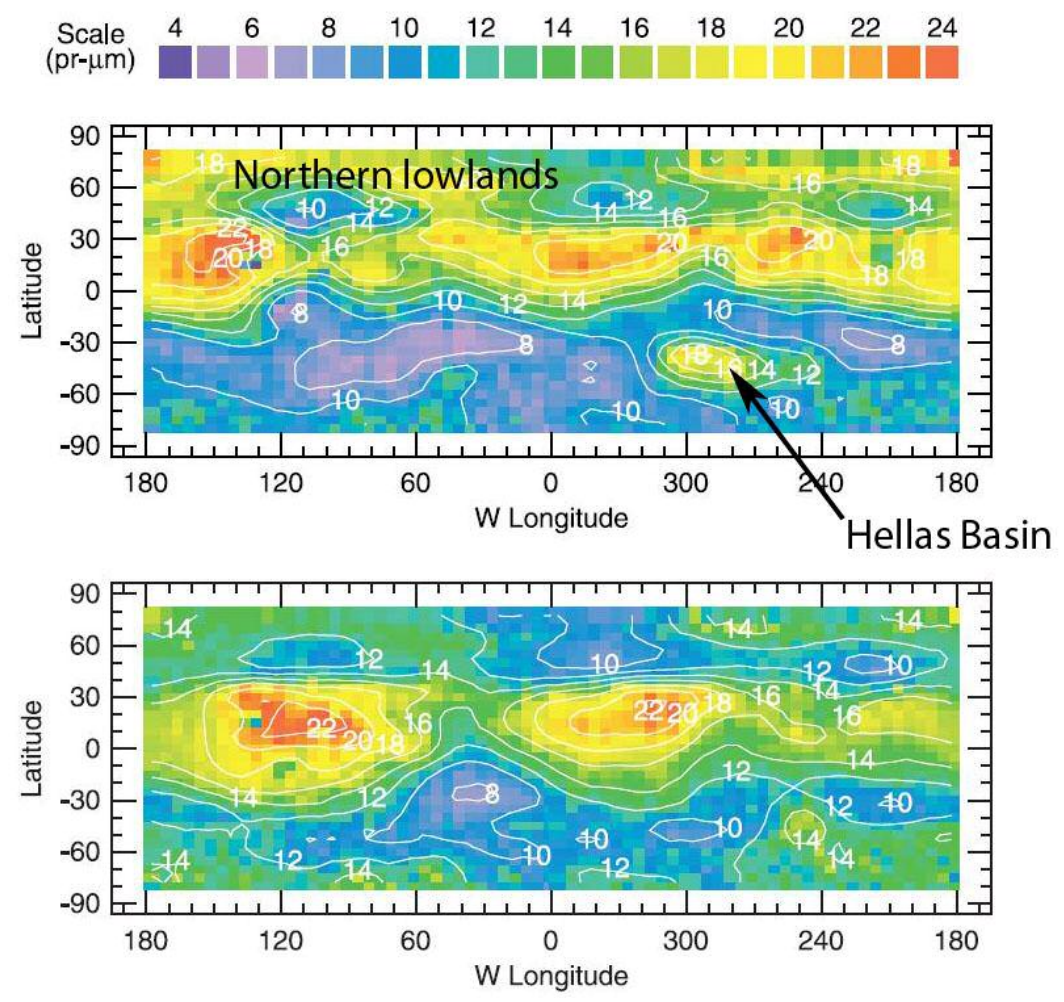

Figure 6 Annual average water column observed over the surface of Mars after the first year of TES observations (Smith 2002). The top panel is a raw result found by averaging observed water contents over a martian year. In the lower panel the water content is corrected for surface pressure. Note that high water column contents associated with locations of low elevation such as Hellas Basin and the Northern Lowlands disappear when water column is corrected to a base level. 
A significant difference between the MAWD results and first martian year of TES results is the presence or absence of a latitudinal gradient in the average water vapor column. MAWD results implied such a gradient did exist and was consistent with a slow supply of water to the south $\mathrm{CO}_{2}$ residual cap that can act as a cold trap to water vapor. TES observations from the first martian year found high water vapor in the southern summer and that a gradient, if present, was not significant. However, TES saw lower water vapor in the second martian year of MGS operation shown by Smith (2004), which was the most significant interannual variability noted by the author.

Another notable TES result was the uniformity of the seasonally averaged water vapor column over both latitudes and longitudes. The lower panel of Figure 6 shows the average water column over the martian globe corrected for surface elevation; in this presentation, high column values over the deep Hellas Basin and over the low northern plains disappear. The average corrected column values still vary around a factor of 2 that is still not explained, though a correlation with albedo was noted. The average corrected column over the globe shown by Smith (2002) is $\sim 20 \mathrm{pr}-\mu \mathrm{m}$ over latitudes 10 to $40^{\circ} \mathrm{N}$ and $\sim 10 \mathrm{pr}-\mu \mathrm{m}$ outside of these latitudes. This variation may seem significant, though even a variation of a factor of a few is much less than the seasonal variation. For example, note the variation in the northern polar regions in Figure 5. Despite this change from $\sim 0$ to $\sim 100 \mathrm{pr}-\mu \mathrm{m}$ during the martian year, the seasonally averaged water column is close to that found at low latitudes which do not experience such extreme seasonal variations. 
There have been many models presented to explain various aspects of the martian water cycle. Here we discuss several models that identify the most significant physical processes in determining the water content of the atmosphere. These models are useful to us as we try to construct a simple scheme to give us a reliable estimate of the amount of water we should expect in the atmosphere during past epochs.

It was recognized early on that the present atmospheric water content is primarily determined by the exchange of water with the polar caps. Davies (1981) proposed such an idea with a model that allowed water to sublime and condense on the surface with residual ice caps at the poles; water vapor then mixed diffusively across the latitudes. Jakosky (1983) had a similar model with water mixing diffusively through the atmosphere. His model also allowed exchange of water between atmosphere and the regolith, where water in the regolith was primarily adsorbed water (layers of water that adhere to solid surfaces). Despite the inclusion of the regolith as an extra water reservoir, Jakosky supported the previous conclusion that the water in the atmosphere is still controlled by polar processes; all non-polar reservoirs were found to respond to the water content determined at the poles. More recent models that use 3-D general circulation models (GCMs) of the atmosphere (e.g. Richardson and Wilson 2002b) still support this conclusion.

Many water cycle models have sought to include a north-south asymmetry in the transport of water in the atmosphere. The motivation for this transport asymmetry has been to explain the north-south gradient suggested in the MAWD results and why water ice is found on the surface only at the north pole. TES results indicate that this gradient 
may not always be present on Mars. Davies (1981) created asymmetry by applying larger diffusivity values to the atmospheric water mixing during the southern summer, arguing the mixing will be enhanced by the dust storm activity associated with this season. Similarly, James (1985) included the global $\mathrm{CO}_{2}$ condensation flow, which is strongest during the southern summer/spring, as another process to enhance the transport of water to the north. Warmer global temperatures at perihelion also act to enhance water transport to the north, as discussed by Clancy et al. (1996). Martian global circulation contains a single Hadley cell that straddles the equator so that water is transported from the summer hemisphere to the winter hemisphere by the upper branch of the cell. When Mars is at aphelion, cooler global temperatures mean water condenses out lower in the atmosphere reducing the amount of water that gets into this upper branch and the effectiveness of the water transport to the south in the present epoch (Clancy et al. 1996). North-south asymmetries have also been proposed to be present in the long-term climate of Mars. The processes stated above for the present martian climate are functions of the present orbit and the relative positions of perihelion and the solstices. These positions are cyclical with no preferred orientation over the history of Mars. Richardson and Wilson (2002a) demonstrate that the north-south asymmetry in the topography of Mars is also capable of enhancing the transport of water to the north due to atmospheric circulation. Jakosky (1993) also stated that the topographic differences create a bias for water transport to the north because seasonal $\mathrm{CO}_{2}$ frost covers the north longer. The higher south polar cap has a lower $\mathrm{CO}_{2}$ frost point temperature which delays the formation of seasonal frost; so any water ice in the south will be available to sublime 
longer, on average, than ice in the north cap. Both of these topographi€ related asymmetries are not cyclical and represent a long term biases in the water cycle of Mars.

An unresolved issue in the water cycle models is the significance of water exchange between the atmosphere and the regolith. It seems certain that the regolith outside the polar regions must be a significant reservoir of water given the ability of the regolith to hold both ground ice and adsorbed water in column densities that are orders of magnitude greater than the atmospheric density. However, it is not clear if these regolith reservoirs are capable of exchanging with the atmosphere on the seasonal timescale in significant quantities. Some water cycle models (Houben et al. 1997; Böttger et al. 2005) have suggested that exchange with the regolith is essential in order to reproduce the observed distribution of the water vapor; without this exchange they found too much water vapor was carried out of the north polar region. However, Richardson and Wilson argue strongly that this regolith exchange is not necessary to reproduce the observed patterns of atmospheric water vapor which they demonstrate with their own GCM. A detailed 1-D model of the exchange of adsorbed water in the regolith with vapor in the atmosphere was done by Zent et al. (1993). While their results showed only a minimal exchange in a diurnal cycle (of the order of $0.75 \mathrm{pr}-\mu \mathrm{m}$ out of $\sim 15 \mathrm{pr}-\mu \mathrm{m}$ in the atmosphere), it would seem entirely possible that there could be a more substantial exchange over the seasons.

It has been noted by a number of authors that water ice would be unstable if it were exposed on the surface at the south pole of Mars in the present epoch. Given that it was first predicted that $\mathrm{CO}_{2}$ would be stable to the lower of the 2 poles (the north), it 
would seem that there should be no cap in the south at all. Davies (1981) claimed water would be unstable in the south based on the high water carrying capacity of the atmosphere in the southern summer. This holding capacity is related to the warm atmospheric temperature profile and this is ultimately because Mars is near perihelion while the south is in summer, raising the insolation and temperatures. Richardson and Wilson (2002b) were able to demonstrate that southern water ice would still be unstable in the present epoch even if southern summer temperatures were not warmer. Richardson and Wilson ran their GCM with water ice exposed in the south and the albedo was adjusted so that peak summer temperatures were less than those in the north. Lower temperatures in the south would normally imply that water ice would become stable at this location, though they found that water ice was still lost from the south in their model, presumably due to asymmetry in the circulation.

Some of the past water cycle models have attempted to explore the aspects of the cycle at other epochs. The presence of low-latitude surface ice during periods of high obliquity was proposed by Jakosky and Carr (1985) as a means to potentially explain the low-latitude valley networks. They calculated that the sublimation of polar ice would saturate at low latitudes, depositing ice on the surface. In another work Jakosky et al. (1993), estimated seasonal sublimation fluxes from polar ice caps over past epochs as a way of estimating the water transport from one pole to the other over the history of Mars. Jakosky et al. (1993) explained the age and extent of polar layered terrains and also the difference in the size of the 2 polar residual caps; the southern cap being smaller due to long-term asymmetry in water transport to the north. The seasonal sublimation fluxes of 
Jakosky et al. (1993) were used as the basis for estimating the atmospheric water content when Mellon and Jakosky (1995) calculated the extent of ground ice during past epochs. The water cycle at other epochs has now been explored with GCMs (Mischna et al. 2003; Mischna and Richardson 2005). GCM results indicate that ice should form on the surface at low latitudes during periods of high obliquity. Also calculated are the atmospheric water contents at these times; an example is shown in Figure 7. Mischna and Richardson (2005) found that the atmospheric water content at high obliquities was sensitive to the location of surface ice. They found that if ice was removed from the surface at the poles at high obliquities, the atmospheric water content was only controlled by the surface ice at low latitudes and was lower by a factor of a few. Note that this scenario does not require the polar reservoir to be completely removed; the formation of a substantial lag deposit over the polar surface icewould be sufficient to impede the exchange with the atmosphere to allow the low-latitude ice to dominate. 

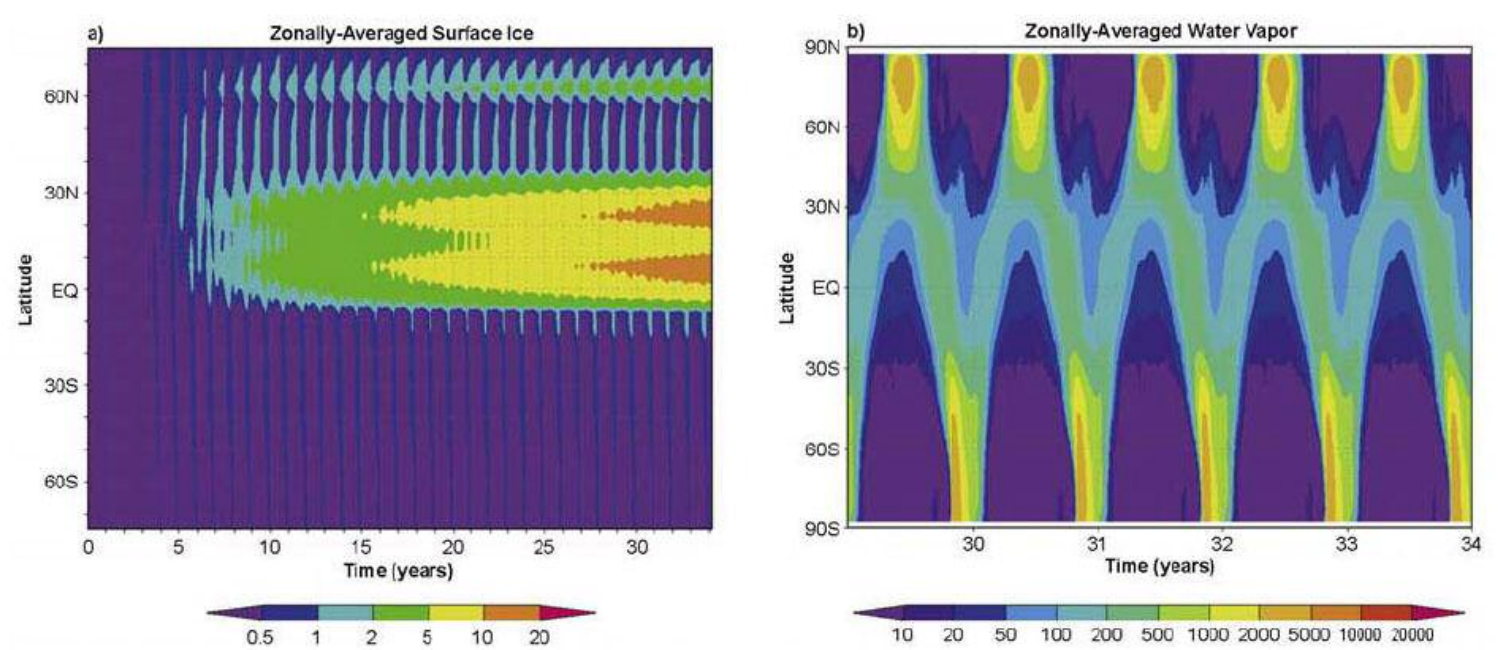

Figure 7 Water cycle results from a GCM at an obliquity of $45^{\circ}$ (Mischna et al. 2003). The left panel (a) shows the deposition and buildup of surface ice soon after the start of the model, ice thickness is in $\mathrm{cm}$. The right panel (b) shows the distribution of the zonal water vapor over the last years of the GCM run, column contents are in pr- $\mu \mathrm{m}$.

\subsubsection{Organization}

We have developed our own set of models to explore the stability of water ice for the present and past epochs. The various components of the model are described in Section 2.2, including the thermal model used to derive temperatures of the surface and subsurface of Mars, and how the water content of the atmosphere is treated. The thermal model used is a 1-D, two-layer model that allows us to include the change in thermal properties with an ice-rich layer. For the most part, our thermal model is similar to other thermal models developed for the near-surface ground of Mars (e.g., Fanale et al. 1986; Haberle and Jakosky 1991; Mellon and Jakosky 1993; Putzig et al. 2005; Schorghofer 
and Aharonson 2005). The components of the model that are different from other models are discussed in more detail in this section. Included in this section are the assumptions we make about the climate in past epochs relevant to the stability of ground ice.

In Section 2.3 we present our results showing the theoretical distribution of stable ground ice for a variety of conditions. In Section 2.3.1 we show the sensitivity of ground ice in the present epoch to various components of the models used and to varying amounts of the atmospheric water vapor. Section 2.3.2 shows the calculated stable ground ice distribution for different orbits representing past martian epochs. First, obliquity is held constant and the position of perihelion is varied, representing the martian orbit over the past few hundred thousands years. Second, effects of the perihelion/aphelion asymmetry are suppressed by circularizing the orbit and obliquity is varied to indicate the extent of the changes in the ground ice distribution over the longer evolution of the martian orbit.

Section 2.4 includes a discussion of how the history of martian ground ice may be reflected in landforms observed from spacecraft. The main results from our work in this project are summarized in the Section 2.5. 


\subsubsection{Glossary}

Below is a list of terms that appear in CHAPTER 2 along with the way in which these terms are used in this dissertation.

Atmospheric water carrying capacity: the average column abundance of water vapor in the atmosphere if the atmosphere is saturated every day of the martian year.

pr- $\mu \mathrm{m}$ : precipitable micron, a measure of the column abundance of water vapor in the atmosphere. This is the thickness of a layer of liquid water that could be formed by condensing all the water vapor in the atmosphere onto a surface.

Crocus date: the time of year when the last $\mathrm{CO}_{2}$ sublimes from the surface at a location and the surface temperature is no longer buffered to the frost temperature.

$\mathbf{L}_{\mathrm{s}}$ : the solar longitude; the angular position of the planet in its orbit, $\mathrm{L}_{\mathrm{s}}=0^{\circ}$ corresponds to the vernal equinox.

Perihelion- $\mathbf{L}_{\mathrm{s}}$ : the solar longitude of the pericenter of the planet's orbit.

Epoch: a period of time that can be described by a particular climate or orbit in the history of a planet.

Diurnal skin depth: the e-folding depth of a sinusoidal diurnal thermal wave; the depth at which the amplitude of temperature variations in the diurnal cycle are 1/e (36.7\%) of the variation amplitude at the surface. 
Near-surface atmosphere: the lowest part of the atmosphere that would be in diffusive with any ice with the ground. It is also part of the atmosphere that is depleted of water vapor due to cold surface temperatures at night.

Equivalent water vapor density: the density found at the surface, based on the atmospheric water column abundance and assuming the column is well mixed vertically.

Effective water vapor density ratio the seasonal average vapor density of the nearsurface atmosphere relative to the equivalent vapor density of the average atmospheric water column at the surface.

Ice-table vapor density: the seasonal average of water vapor density at the top of the ice that is permanently present in the ground.

Adsorbed water: thin layers of water that adhere to solid surfaces.

GCM: General Circulation Model.

MOLA: Mars Orbital Laser Altimeter, an instrument onboard the Mars Global Surveyor spacecraft.

TES: Thermal Emission Spectrometer, an instrument onboard the Mars Global Surveyor spacecraft.

GRS: Gamma Ray Spectrometer, an instrument onboard the Mars Odyssey spacecraft.

Terrain softening: an empirical term to describe regions where topographic features on the surface appear to be relaxed; craters are poorly preserved and blocky, angular features are infrequent or absent. Terrain softening is observed in mid to high latitudes of Mars. 
Mantle deposit: a layer of material that appears as a blanket over the surface.

Dissected mantle: regions of the mantle deposit where the smooth mantle surface is broken.

Lag deposit: a layer of non-volatile material that is left behind on a surface as volatile material sublimes away. 


\subsection{Model Construction}

In this section we discuss the model that is used to determine where ground ice is stable on Mars. We discuss briefly and cite references those aspects of the model that have been described before in the literature. We discuss in more detail model aspects that are new and that we have derived ourselves.

Finding where ground ice is stable to vapor diffusion is a two-step process. The first step requires finding the average water vapor density over ground ice, or the icetable vapor density, as a function of depth to ground ice for any location on Mars. Vapor density is found from the temperature of the top of the ground ice. A near-surface thermal model is used to calculate the ice-table vapor densityover a martian year for a wide range of locations and surface properties. The second step is to find the average vapor density of the atmosphere just above the surface. Ground ice is considered stable to diffusion with the atmosphere when seasonally averaged water vapor densities of the atmosphere and the ground ice are equal. While vapor diffusion is not considered explicitly in the models, it is the underlying assumption for the approach that is used. Section 2.2.1 contains a discussion of vapor diffusion and why average vapor densities can be used to estimate depth to stable ice. A description of the thermal model is given in 2.2.2. Subsections describe the convergence algorithm used to accelerate the finding of a solution that is independent of initial conditions, and the effects of the atmosphere on the thermal model. In Section 2.2.3 we describe a scheme that allows the water vapor 
density in the part of the atmosphere that is in diffusive contact with ground ice to have a lower water content than the rest of the atmospheric column for a particular location. How the thermal model results are used to find the depth to stable ice is described in Section 2.2.4. We describe how the thermal model results are interpolated from a grid of discrete locations and property values to particular locations on Mars with particular properties in Section 2.2.4.2. Assumptions that we make about the martian climate in past epochs are discussed in Section 2.2.5. Both the responses of $\mathrm{CO}_{2}$ and $\mathrm{H}_{2} \mathrm{O}$ in the atmosphere to obliquity are discussed.

Our objective is to produce a model that makes reasonable reproduction of maps of ground-ice distribution as has been published previously (Schorghofer and Aharonson 2005; Mellon et al. 2004; Mellon and Jakosky 1993). These previous ground-ice distributions are, to first order, also consistent with the ground ice observed by the Mars Odyssey GRS (Feldman et al. 2004b; Boynton et al. 2006). We then use our model to find the extent of ground ice as a response to climate change driven by orbit. At present, the limit of the martian ground ice is in the mid latitudes where the gradient of average surface temperature is about $1 \mathrm{~K}$ per $1^{\circ}$ latitude. We have designed our models and interpolation schemes used to generate our maps so that the annuallyaveraged surface temperature derived at any point of Mars is within $1 \mathrm{~K}$ of an ideal solution; where an ideal solution would be a map produced with a model that is unaffected by initial conditions, interpolation, temporal resolution or spatial resolution. An error less than $1 \mathrm{~K}$ would give an error in the ground ice distribution of less $1^{\circ}$ latitude, or less than a single pixel in our grid. In order to achieve this, temporal and 
spatial resolutions in the thermal model and interpolation schemes have been designed so that each process is accurate to $0.1 \mathrm{~K}$, an order of magnitude better than the stated goal in order to ensure that results are not affected by model artifacts.

We recognize that there are limitations on the ability of any model to reproduce martian surface temperatures. Some assumptions in the models are poorly determined or are known to vary with time, such as the opacity of the atmosphere. Variations in these assumptions can produce deviations that exceed the stated goal, but they are not artifacts of the model construction.

\subsubsection{Vapor Diffusion}

The diffusion of water vapor is not considered explicitly in our models, however vapor diffusion between near-surface ground ice and the atmosphere is assumed to the main process controlling the stability of ground ice.

It has been shown that it is reasonable to consider ground ice to be stable to diffusion with the atmosphere when the seasonally averaged near-surface atmospheric vapor density and ice table vapor density are equal. Leighton and Murray (1966) originally suggested that ice would stable at depth when the average vapor pressure at depth was the same as the near-surface atmosphere. Mellon and Jakosky (1993) and Schorghofer and Aharonson (2005) demonstrate that the depth to stable ice using just the average vapor pressures (or densities) is the same as the depth that is found with models that explicitly include vapor diffusion. The advantage of using average vapor pressures 
or densities is that they can be calculated with a thermal model without vapor diffusion which makes the procedure much less computationally intensive.

When ground ice is stable there is no net vapor flux into or out of the ground over a full season. As explained by Schorghofer and Aharonson (2005) the key assumption that makes this possible is that the average seasonal flux in the system can be approximated well with the average vapor gradient. The water vapor flux at any time is found by the product of the vapor gradient with a diffusion coefficient, $\operatorname{Flux}_{z}=D(T) \frac{d n}{d z}$

$D$ is the diffusion coefficient, $T$ is the temperature, $n$ is the vapor density and $z$ is the vertical coordinate. The coefficient is a function of temperature and it is not intuitive that the vapor flux averaged over the seasons is just proportional to the average of the vapor gradient. Schorghofer and Aharonson (2005) point out that the variations in the coefficient and the vapor gradient over the seasons are not coupled. They find that it is valid to estimate the average flux over the seasonal by using the averages of the diffusion coefficient and the vapor gradient, i.e.,

$$
\left\langle\operatorname{Flux}_{z}\right\rangle=\left\langle D(T) \frac{d n}{d z}\right\rangle \approx\langle D(T)\rangle\left\langle\frac{d n}{d z}\right\rangle .
$$

When flux can be approximated this way, the seasonal flux will be zero when the average vapor gradient is zero. The atmosphere and the top of the ground ice are the two boundaries of the system considered here, so an average vapor density gradient of zero implies that the atmosphere and the ground ice have the same average vapor density. 
Like Mellon and Jakosky (1993) and Schorghofer and Aharonson (2005), we can also show our own results of depths to stable ground ice found with vapor diffusion models. We did this some years ago with early versions of the model we present here. In Figure 8 we show the depth to stable ice we estimated with different approaches, both including explicit vapor diffusion and also using the seasonal averages. The one-layer and two-layer thermal model depths shown in Figure 8 are estimated using the assumption the ice is stable if the ice-table vapor density is equal to the density in the atmosphere. The one-layer thermal model assumes the thermal properties are uniform with depth which was the approach used by Mellon and Jakosky (1993). The two-layer thermal model assumes the ground has the properties of high-thermal inertia where ice is present which was the approach used by Mellon et al. (2004). At selected latitudes, the depth to stable ground ice is also determined with a model that includes thermal and vapor diffusion explicitly; this model was a computationally expensive model so this was only performed at a few locations. The vapor diffusion model partitions the amount of water at any particular depth between 3 phases: vapor, ice and adsorbed water. Water vapor is the only mobile phase and transport is calculated by the vapor density gradient and the temperature-dependent diffusion coefficient. The presence of adsorbed water in the regolith dampens changes in the vapor density; most of the water vapor transported by the vapor density gradient goes into the adsorbed phase and only slowly changes the vapor density. In this implementation of the model, the vapor diffusion is resolved seasonally but not diurnally. In later sections we will discuss a process that depletes the amount of water in the lowest part of the atmosphere due to the diurnal cycle. When the 
diurnal cycle is not resolved in the vapor diffusion process the effect of this vapor depletion is not shown; nor is effect of vapor depletion (as discussed in Section 2.2.3) considered in the stable ice depth determined with thermal models in Figure 8. The thermal properties of each depth layer are allowed to be a function of the amount of ice present in the layer. The amount of ice in any layer is allowed to increase or decrease in response to temperature and vapor density gradients. In Figure 8 the albedo and thermal inertia of ice-free ground at each latitude is the average albedo and thermal inertia determined by remote sensing in these latitude bands. Note that the depth to stable ground ice found with vapor diffusion is the same as the depth found with the two-layer thermal model.

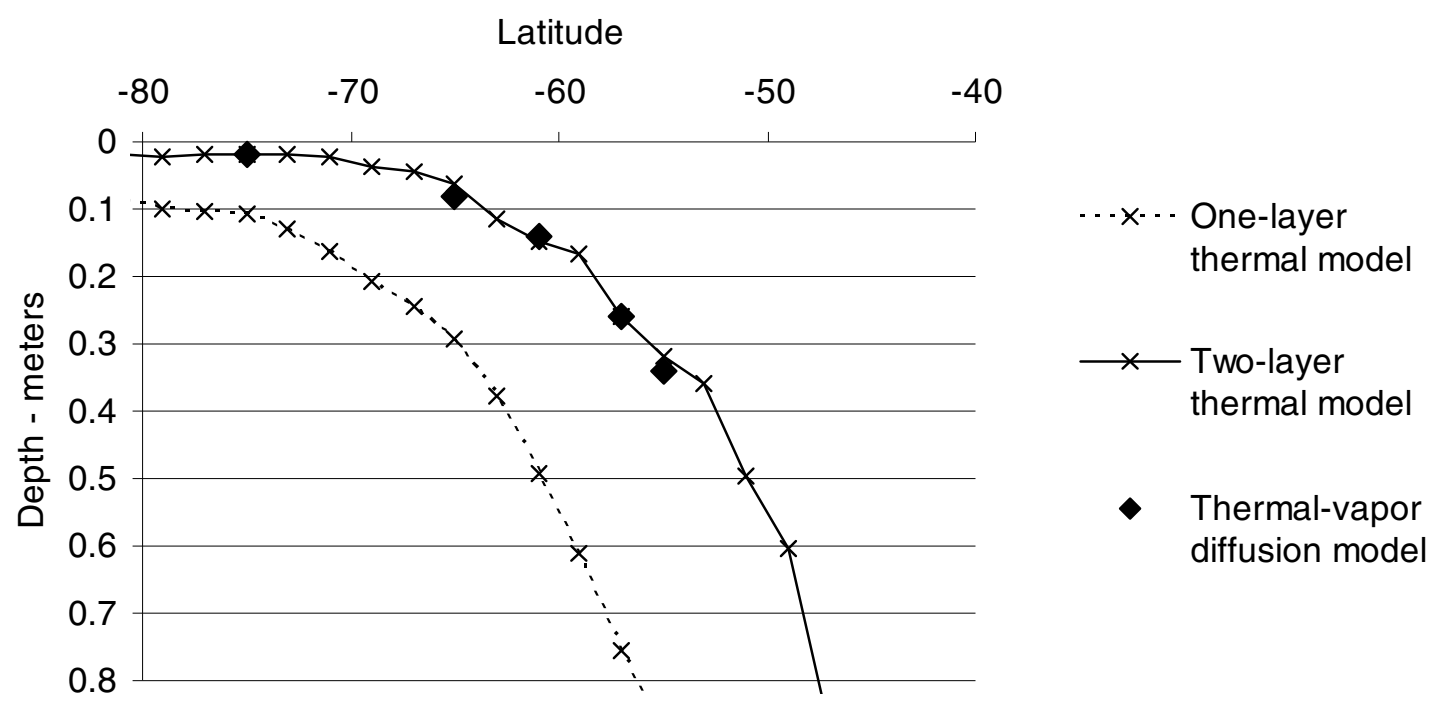

Figure 8 Depth to stable ice estimated with a variety of models, as a function of latitude. The onelayer and two-layer thermal model depths are estimated using the assumption that ice is stable when the ice-table vapor density is equal to the density in the atmosphere. At selected latitudes, the depth to stable ground ice is also determined with a model that includes thermal and vapor diffusion explicitly and coupled together. The thermal properties of each depth layer are allowed to be a function of the amount of ice present in the layer. Remote sensing results are used for albedo and thermal inertia of each latitude band. 
By assuming that the net seasonal vapor flux can be approximated with average vapor densities found using a thermal model with a fixed setup implicitly assumes that there is no significant loss or gain of ice from or onto the ice table during the season. Also, the variation in thermal conductivity as a function of temperature is not considered. However, while either of these effects may modify the depth at which ground ice is stable, they should not change where ground ice is stable. The reason for this is that both of these effects will be most pronounced when ice is shallow. It is 'easier' for ice to be stable deeper below the surface than close to the surface, that is, the average vapor density over the ground ice is less when ice is deeper, reaching an effective minimum when the ice table is a seasonal skin depth or two below the surface. When ice is deeper, there is less variation in temperature with the seasonal cycle. The temperature of deep ground ice and the vapor density over the ice is then effectively just the average throughout the year and effects due to seasonal changes will be negligible.

\subsubsection{Thermal Model}

Changes in the ground temperatures in our model are calculated with a semiimplicit, Crank-Nicholson, 1-D thermal diffusion scheme. The topmost layer has zero thickness, the temperature of which is the model surface temperature and is found by the instantaneous energy balance at any time. The thickness of the next layer is set to 0.2 of the diurnal skin depth and the thickness of each following layer is 1.2 times that of the 
layer before. The lower boundary of the thermal model is made deeper than any significant temperature variation. Our model used 50 layers to ensure no temperature variations at the base of the model. Temperatures are associated with the mid-points of each layer.

At the top boundary the temperature is found by the energy balance of insolation, thermal radiation, sensible heat exchange, conduction and latent heat of $\mathrm{CO}_{2}$. The idealized energy balance at the surface is shown in the equation below

$$
I_{s}(1-A)+I R_{a t m o}+S+k\left(\frac{\partial T}{\partial z}\right)_{z=0}+L_{C O_{2}} \frac{d m_{c o_{2}}}{d t}=\varepsilon \sigma T_{\text {surf }}^{4}
$$

$I_{s}$ is the insolation flux transmitted through the atmosphere as a function of distance to and zenith angle of the Sun, $A$ is albedo, $I R_{\text {atmo }}$ is thermal radiation from the atmosphere, $\varepsilon$ is emissivity, $S$ is the sensible heat exchange with the atmosphere $\sigma$ is the StefanBoltzmann constant, $T_{\text {surf }}$ is surface temperature, $k$ is thermal conductivity, $T$ is temperature, $z$ is the depth coordinate, $L_{\mathrm{CO} 2}$ is the latent heat for the sublimation of $\mathrm{CO}_{2}$, $m_{\mathrm{CO} 2}$ is the mass per area of $\mathrm{CO}_{2}$ on the surface and $t$ is time. The transmission of insolation, thermal radiation and sensible heat exchange with the atmosphere are calculated in a way based on Haberle and Jakosky (1991), as described in Section 2.2.2.2. This equation includes the terms used for when $\mathrm{CO}_{2}$ frost is both present and absent; the presence of frost on the surface buffers the temperature, when net energy flux at the surface then modifies the amount of frost present. In practice, this equation is discretized to be used in the model. When there is no $\mathrm{CO}_{2}$ frost the equation becomes an implicit solution of surface temperature $\left({ }^{j+1} T_{0}\right)$, as shown below. 
${ }^{j+1} I_{s}(1-A)+{ }^{j+1} I R_{a t m o}+{ }^{j+1} S+\frac{k_{0 \rightarrow 1}}{\Delta z_{0 \rightarrow 1}}\left({ }^{j+1} T_{1}-{ }^{j+1} T_{0}\right)=\varepsilon \sigma\left({ }^{j} T_{0}{ }^{4}+4{ }^{j} T_{0}^{3}\left({ }^{j+1} T_{0}-{ }^{j} T_{0}\right)\right)$.

Upper indices indicate which time step is associated with a quantity, $j$ is for the present and $j+1$ is for the future time step. Lower indices indicate the associated depth layer, 0 is the surface and $O \rightarrow 1$ indicates a property associated between midpoints of adjacent layers 0 and 1 . Note that the term for thermal radiation from the surface is linearized so that it can be solved implicitly and is accurate as long as temperature difference between time steps is small. Note that solving for ${ }^{j+1} T_{0}$ requires simultaneously solving for ${ }^{j+1} T_{1}$ and the rest of the model temperatures. This solution is found as part of the Crank-Nicholson scheme.

When frost is present on the ground, the surface temperature is buffered at the frost point by the latent heat of $\mathrm{CO}_{2}$ frost. The frost point is at $\sim 148 \mathrm{~K}$ for a pressure of $610 \mathrm{~Pa}$. The energy balance equation then becomes a semi-implicit calculation of the change in mass of $\mathrm{CO}_{2}$ frost,

$L_{\mathrm{CO}_{2}}\left({ }^{j+1} m_{\mathrm{CO}_{2}}-{ }^{j} m_{\mathrm{CO}_{2}}\right)=\frac{\Delta t}{2}\left(\begin{array}{l}\varepsilon \sigma\left({ }^{j} T_{0}^{4}+{ }^{j+1} T_{0}^{4}\right)-\left({ }^{j} I_{s}+{ }^{j+1} I_{s}\right)(1-A)-{ }^{j} I R_{\text {atmo }}-{ }^{j+1} I R_{\text {atmo }}- \\ { }^{j} S-{ }^{j+1} S-\frac{k_{0 \rightarrow 1}}{\Delta z_{0 \rightarrow 1}}\left({ }^{j} T_{1}-{ }^{j} T_{0}+{ }^{j+1} T_{1}-{ }^{j+1} T_{0}\right)\end{array}\right)$

The values for the albedo and emissivity of the surface are changed whenever $\mathrm{CO}_{2}$ frost is present. The time step used is $1 / 100^{\text {th }}$ of the martian day. The exception to this time step is when $\mathrm{CO}_{2}$ frost formed or disappeared from the surface; in this case a partial time step is used to determine the new temperature profile at the instant that $\mathrm{CO}_{2}$ frost forms or disappears. 
Below the top boundary, thermal diffusion between the layers follows the form of $\rho c \frac{\partial T}{\partial t}=\frac{\partial}{\partial z}\left(k \frac{\partial T}{\partial z}\right)$

where $\rho$ is density, and $c$ is specific heat. When this equation is discretized for use in the semi-implicit model with variable spacing, it becomes a little more complicated,

$(\rho c)_{i}\left({ }^{j+1} T_{i}-{ }^{j} T_{i}\right)=\frac{\Delta t}{2 \Delta z_{i}}\left(\begin{array}{l}\frac{k_{i \rightarrow i+1}}{\Delta z_{i \rightarrow i+1}}\left({ }^{j} T_{i+1}-{ }^{j} T_{i}+{ }^{j+1} T_{i+1}-{ }^{j+1} T_{i}\right)+ \\ \frac{k_{i-1 \rightarrow i}}{\Delta z_{i-1 \rightarrow i}}\left({ }^{j} T_{i-1}-{ }^{j} T_{i}+{ }^{j+1} T_{i-1}-{ }^{j+1} T_{i}\right)\end{array}\right)$

where indices are used the same way as before for the top boundary condition.

No geothermal gradient is considered in these models. The effect of the geothermal heat is negligible for the temperatures near the surface where we are interested here. For example, if a surface had a temperature of $195 \mathrm{~K}$ and an additional heat flux $0.03 \mathrm{Wm}^{-2}$ was applied (an estimated martian geothermal heat flux), the temperature would only increase by $\sim 0.02 \mathrm{~K}$.

The attenuation of insolation and thermal radiation from the atmosphere are calculated by one of two different atmospheric models that are described in Section 2.2.2.2. The differences between the 2 atmospheremodels are the level of detail in the model and the computer run time required.

A sample of the thermal model output is shown in the figures that follow; the inputs to the model used to generate these figures were a latitude of $70^{\circ} \mathrm{N}$, a thermal inertia of 300 S.I. units $\left(\mathrm{J} \mathrm{m}^{-2} \mathrm{~K}^{-1} \mathrm{~s}^{-1 / 2}\right)$, an albedo of 0.20 and an ice depth of $7.9 \mathrm{~cm}$. Figure 9 shows the average and range of surface temperatures for every day of the 
martian year, as a function of solar longitude $\left(\mathrm{L}_{\mathrm{s}}\right)$ which is a measure of the position of the planet in its orbit. Figure 10 shows the local time of sunrise and sunset for the same model to help visualize the seasons of the model in Figure 9. Figure 11 shows the temperature profile in the subsurface over the martian year. Significant constant values used by the model are shown in Table 1.

\begin{tabular}{|c|c|c|}
\hline Parameter & Value & Source (where applicable) \\
\hline $\begin{array}{l}\text { Ground specific heat } \\
\text { capacity }\end{array}$ & $840 \mathrm{~J} \mathrm{~kg}^{-1} \mathrm{~K}^{-1}$ & Typical value for silicate mineral \\
\hline $\mathrm{CO}_{2}$ frost albedo & 0.65 & $\begin{array}{l}\text { (Mellon and Jakosky 1993; Paige and } \\
\text { Ingersoll 1985) } \\
\text { (cf } 0.6 \text { used by (Mischna et al. 2003) }\end{array}$ \\
\hline $\mathrm{CO}_{2}$ emissivity & 0.8 & $\begin{array}{l}\text { (Mellon and Jakosky 1993; Mischna et al. } \\
\text { 2003) }\end{array}$ \\
\hline Bare ground emissivity & 1.0 & \\
\hline Latent heat of $\mathrm{CO}_{2}$ & $590000 \mathrm{~J} \mathrm{~kg}^{-1}$ & \\
\hline $\begin{array}{c}\text { Thermal conductivity of } \\
\text { ice-rich layer }\end{array}$ & $2.5 \mathrm{~W} \mathrm{~m}^{-1} \mathrm{~K}^{-1}$ & \\
\hline $\begin{array}{c}\text { (Specific heat) } * \text { (density) } \\
\text { of ice-rich layer }\end{array}$ & $2 \mathrm{e} 6 \mathrm{~J} \mathrm{~m}^{-3} \mathrm{~K}^{-1}$ & \\
\hline
\end{tabular}

Table 1 Summary of parameter values used for thermal model and determining ice depth. The properties associated with $\mathrm{CO}_{2}$ are assigned to the surface whenever the model $\mathrm{CO}_{2}$ condenses onto the surface. 


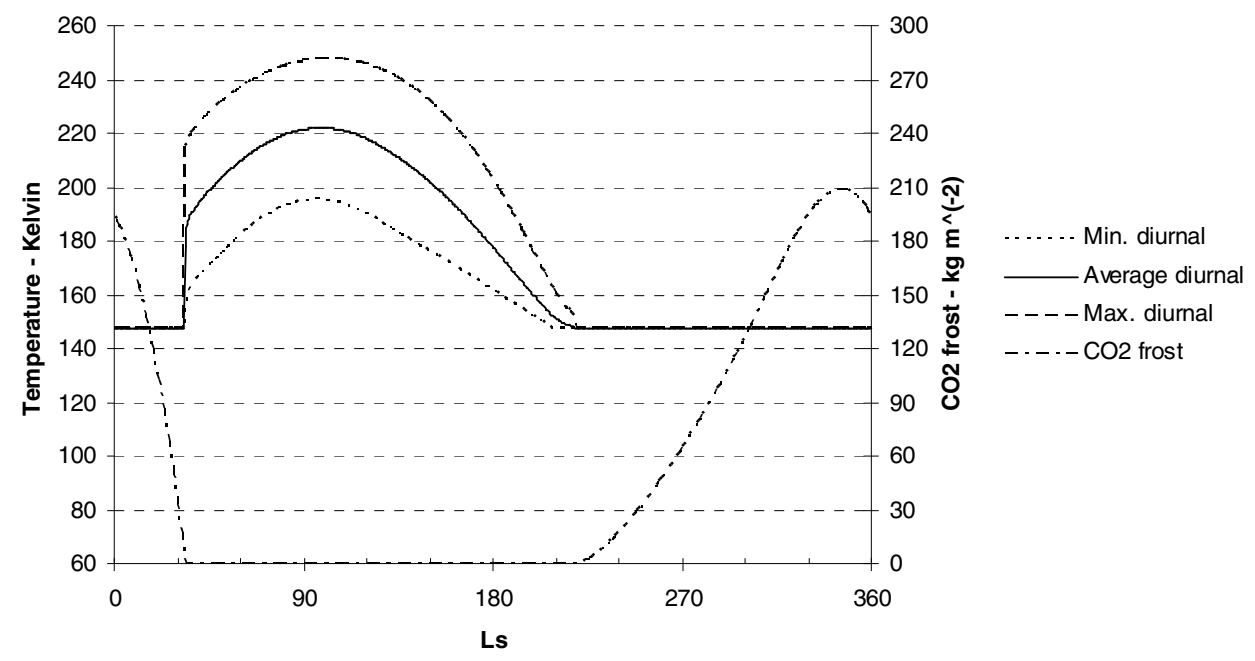

Figure 9 Sample output from thermal model. Results shown are based on a model at $70^{\circ} \mathrm{N}$, thermal inertia of 300 S.I. units $\left(\mathrm{J} \mathrm{m}^{-2} \mathrm{~K}^{-1} \mathrm{~s}^{-1 / 2}\right)$, an albedo of 0.20 and ground ice at $7.9 \mathrm{~cm}$. Shown are the average and range of surface temperatures as a function of $L_{s}$. Over the winter the surface temperature is buffered at the frost point and seasonal $\mathrm{CO}_{2}$ frost builds up.



Figure 10 Sunrise and sunset times as a function of $L_{s}$ for a location at $70^{\circ} N$ latitude. These results are for the same latitude as the model results in Figure 9. 


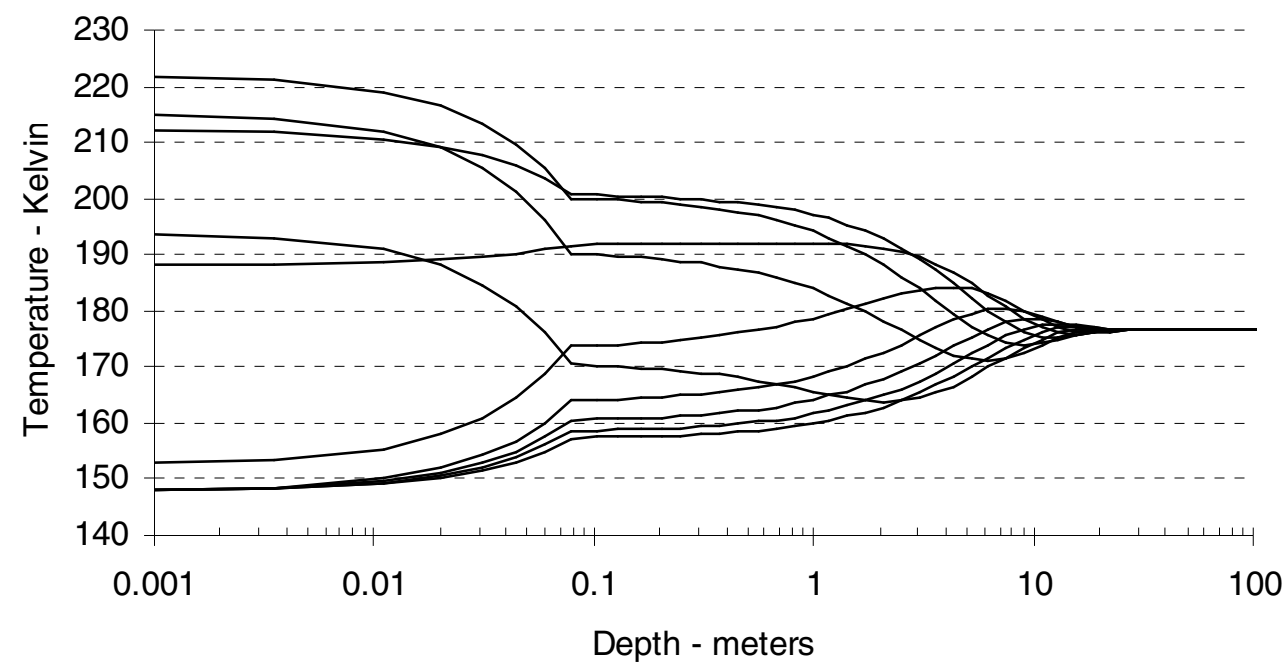

Figure 11 Sample seasonal temperature profiles for the model from Figure 9. Average diurnal temperature profiles are shown for ten evenly-spaced days from the martian year. By taking the diurnal average, the diurnal thermal wave is suppressed so that the figure only shows the seasonal wave. The temperature discontinuity at $7.9 \mathrm{~cm}$ is due to the presence of ground ice and the change in thermal conductivity; heat flux is continuous across this boundary.

\subsubsection{Convergence}

A convergence algorithm was developed to efficiently find a solution to the temperature cycle that repeats to within an acceptable limit that is independent of the initial conditions.

In order to illustrate this problem, the twpanels in Figure 12 below show the average annual surface temperature for each year the thermal model was run for two scenarios. In the first scenario the model was run for an extended period of time without any convergence and in the second scenario convergence was used. The input parameters for the model run are the same as those used to produce the sample results shown in 
Figure 9. The average surface temperature of the model without convergence slowly approaches the same average surface temperature that is found rapidly when the model includes convergence. After 200 model years, the average surface temperature of the model without convergence is still $0.4 \mathrm{~K}$ different from the average temperature found with just several model years when using convergence. To check the accuracy of the average temperature found with the convergence scheme, a model was run for another 100 model years from the last year of the model run that included convergence. In 100 model years, the average surface temperature only varied another $0.0002 \mathrm{~K}$, well within our tolerable limits. 



Figure 12 The changes in annual average surface temperatures, with and without the convergence algorithm. The inputs for the model runs are the same as used in Figure 9. 
The convergence scheme we developed is based on the assumption that if diffusion is the only mechanism that transports heat within the ground and the thermal diffusion coefficient is constant with time, then the profile of seasonally averaged temperatures should be constant with depth in the absence of a geothermal gradient. When running thermal models to calculate where ground ice is stable on Mars, the ground is started with an isothermal profile of $200 \mathrm{~K}$. For each year simulated, the average temperature for each depth layer is calculated. The difference between this average temperature of the layer and the average temperature of the surface for this year is applied to the temperature profile at the instant at the end of the model year. Each model is run 3 times; the thermal model is run for a full year with the convergence algorithm applied at the end of the year. After this, 3 more years are simulated without convergence. If the average annual surface temperature has varied less than an acceptable limit over these 3 years, then the model is considered to be in a repeatable state and an acceptable solution has been found; an acceptable limit of $0.01 \mathrm{~K}$ was used for this project. If the solution is acceptable, the model then simulates one more year to record, calculate and average the temperatures and vapor density over ice for all the layers. If the average annual surface temperature varies more than the acceptable limit in the 3-year run, then the average surface temperature from the last year is used to converge the temperature profile at the instant of the end of the model year, as described above. The model simlates another 3 years and will again test if the annual average surface temperature repeats within the acceptable limit. This cycle will run until the acceptable limit is reached. 
Once a solution that repeats within the acceptable limit is found, the variations in the profile of annually averaged temperatures of each layer are typically less than 0.001 K. Checking for a uniform profile in average temperature proved to be a useful way to check the code was doing what it should. This scheme used usually converges rapidly. At low latitudes, a stable solution is often found after just the initial 3-year run. Convergence is slowest at high latitudes and when ground ice is close to the surface.

\subsubsection{Atmosphere}

The effect of the atmosphere in thermal models of the surface of Mars are perhaps the most complicated and yet least significant parts of the model. The atmosphere attenuates incoming insolation and it also is the source of additional thermal radiation received at the ground surface. The attenuation of insolation does not quite cancel out the extra thermal radiation, usually producing a small increase in the energy flux received at the surface. However, despite the atmosphere being a relatively minor component of the energy budget for the surface thermal model, the atmosphere is the most complicated to calculate accurately and uses most of the computer time.

We have used several atmospheric models in the course of this project. As a base, we used a detailed atmospheric model that includes radiative transfer subroutines from the Ames Mars General Circulation Model, kindly provided by Robert Haberle. These routines are the same as those used before to determine thermal inertia (Haberle and Jakosky 1991; Mellon et al. 2004; Putzig et al. 2005). We then produced a simplified 
atmospheric model that includes enough detail to reproduce the surface temperatures. We have taken some short cuts to speed up the run time which are described in following paragraphs. Average temperatures are within a fraction of a Kelvin of the results with the full detailed model, but thesimplified model is quicker to run by an order of magnitude allowing us to run or re-run more models. We also used the " $2 \%$ assumption" for the atmospheric contribution to thermal energy received at the surface for the sake of comparison; this model has been used extensively over the last few decades. The " $2 \%$ assumption" is that the thermal flux received at the surface over a particular day is $2 \%$ of the noon time insolation of that day, or $2 \%$ of the thermal radiation from the ground if sun does not rise that day.

We run the full, detailed Ames modelfor ene rgy transfer through the atmosphere over ground with typical values of thermal inertia and albedo for the surface of Mars found by Putzig et al. (2005). These models are run over a range of latitudes to encompass the range of conditions typically found on the surface of Mars. Then a simple model of the atmosphere is constructed, leaving a number of parameters that can be tuned to match the results of the detailed model.

Radiative transfer in our simple atmosphere model is considered in 3 bands: a simple 2-stream approximates the transmission of visible insolation (simple isotropic scattering is used), $15-\mu \mathrm{m}$ radiation is calculated using the $\mathrm{CO}_{2}$ equivalent width by Pollack et al. (1981) converted to effective emissivities across the band, and a grey atmosphere is used for thermal infrared radiation outside the $15-\mu \mathrm{m}$ band. A reduced number of layers of the atmosphere are used in our simple model; while the Ames model 
includes up to 30 layers we use just 5. The $\mathrm{CO}_{2}$ band equivalent width is updated using average diurnal temperatures, not every time step and no fine-meshing is applied as it is in the Ames radiative transfer model. These modifications significantly speed up the calculation without adversely affecting the radiation received at the surface.

The scheme used in our simple modelto calculate temperatures and fluxes within the atmosphere is the same as that used in the detailed Ames model, as used by Haberle and Jakosky (1991); it is described just briefly here. Changes to the temperatures of the atmospheric layers are found by the conservation of energy and the difference in the radiation fluxes across each layer. The amount of visible insolation absorbed in the layer is estimated from the 2-stream approximation. Infrared fluxes at any level within the atmosphere (both within and out of the $15-\mu \mathrm{m}$ band) are found by integrating the contribution from all atmospheric layers (and ground in the case of upwelling thermal radiation). The thermal flux contribution from any layer to any level is determined by the change in the effective emissivity across that layer as viewed from the level. Corrections to the atmospheric temperature profile are made for both convection and sensible heat flux. The energy fluxes calculated at the base of the atmosphere are then used in the thermal model to calculate surface temperature of the ground.

The tunable parameters in our simple version of the atmosphere model are: 1 . the total optical depth of the atmosphere in the visible (due to scattering and absorption), 2. the single-scattering albedo (ratio of amount of light scattered to light scattered and absorbed), and 3. the ratio of the infrared and visible optical depths of the atmosphere. For the current climate of Mars, the values of these parameters are tuned to match the 
results of an atmosphere with $610 \mathrm{~Pa}$ and an optical depth $(\tau)$ of 0.3 , which are typical values used in the Ames Mars GCM (e.g., Haberle et al. 1993). When the detailed Ames atmosphere model is run with different atmospheric thicknesses - such as for low obliquities and different elevations - the value of the optical depth used is also changed to maintain the same ratio of optical depth to atmospheric pressure. The values of the tunable parameters used for various atmospheric thicknesses in this project are shown in Table 2. Values used were found by minimizing the differences between the average surface temperatures found with the simplified model and the detailed Ames model for the range of conditions described before.

\begin{tabular}{|c|c|c|c|}
\hline Model & $\begin{array}{c}\text { Total visible } \\
\text { optical depths }\end{array}$ & $\begin{array}{c}\text { Ratio of infrared and } \\
\text { visible optical depth }\end{array}$ & $\begin{array}{c}\text { Single Scattering } \\
\text { Albedo }\end{array}$ \\
\hline ob25,e09,p610 & 0.16 & 0.7 & 0.86 \\
\hline ob25,e09,p910 & 0.23 & 0.76 & 0.87 \\
\hline ob25,e09,p409 & 0.12 & 0.76 & 0.905 \\
\hline ob20,e00,p180 & 0.055 & 0.57 & 0.86 \\
\hline ob15,e00,p035 & 0.025 & 0.12 & 0.86 \\
\hline ob10,e00,p010 & 0.0125 & 0.12 & 0.86 \\
\hline
\end{tabular}

Table 2 Values of tunable atmospheric parameters found for different atmospheric thickness and different orbits used in this project. The first parameters shown were used for most of the maps generated. The following two sets of parameters, "ob25,e09,p910" and "...p409," were used to test the effect of surface pressure (and elevation) on thermal model results in the present epoch. The last 3 models listed are used for low-obliquity models when we assume more of the $\mathrm{CO}_{2}$ in the atmosphere has condensed onto perennial caps. For high obliquities we assume the surface pressure is close to the present epoch and use the first set of parameters; see Section 2.2.5.1 for discussion of $\mathrm{CO}_{2}$ as a function of orbit. 
Our simplified atmospheric model would perhapsbe too simple if we were studying the atmosphere itself, but they seem reasonable when we are primarily interested in the ground surface. The values of the tunable parameters are of the same order as corresponding values used in the detailed Ames model, see values for model "ob25,e09,p610" in Table 2. The tuned visible optical depth (0.16) is about half that used for the typical martian atmosphere in the detailed model $\left(\tau_{\mathrm{vis}} \sim 0.3\right)$. This difference is related to the fact that our treatment used a simple isotropic scattering which would enhance the ability of atmospheric dust to increase the planetary albedo with respect to the detailed models; Pollack et al. (1979b) found martian dust scatters 10 times as much light forward as backward. The value of our tuned single-scattering albedo is close to the value in the detailed model; Pollack et al. (1979b) found a single-scattering albedo of 0.86. Our ratio of infrared to visible optical depths (0.7) is a little higher than the effective ratio used by the detailed model $(\sim 0.5)$.

Figure 13 shows the variation of average surface temperatures between different atmospheric models of ground with typical surface properties (bright and dusty, or dark and rocky) over different latitudes. The significance of the average surface temperature is that it is one of the main factors that determine whether ground ice will be present at a particular location, though it in itself does not indicate how deep ice may be. Note there is more variation in the temperatures between atmospheres with different dust opacities than between temperatures of our simple tuned model and the more complex Ames model. Note also that dust opacity varies with time and is poorly constrained on the real Mars. The tuned model gave a very good fit to the $\tau=0.3$ detailed model, by design; 
differences in the average surface temperature are much less than a degree for all latitudes and all properties shown. We found that our simplified model gave us relatively quick accurate results and allowed us to make modifications and test more scenarios. This model could probably be simplified and sped up further if it were really required. 

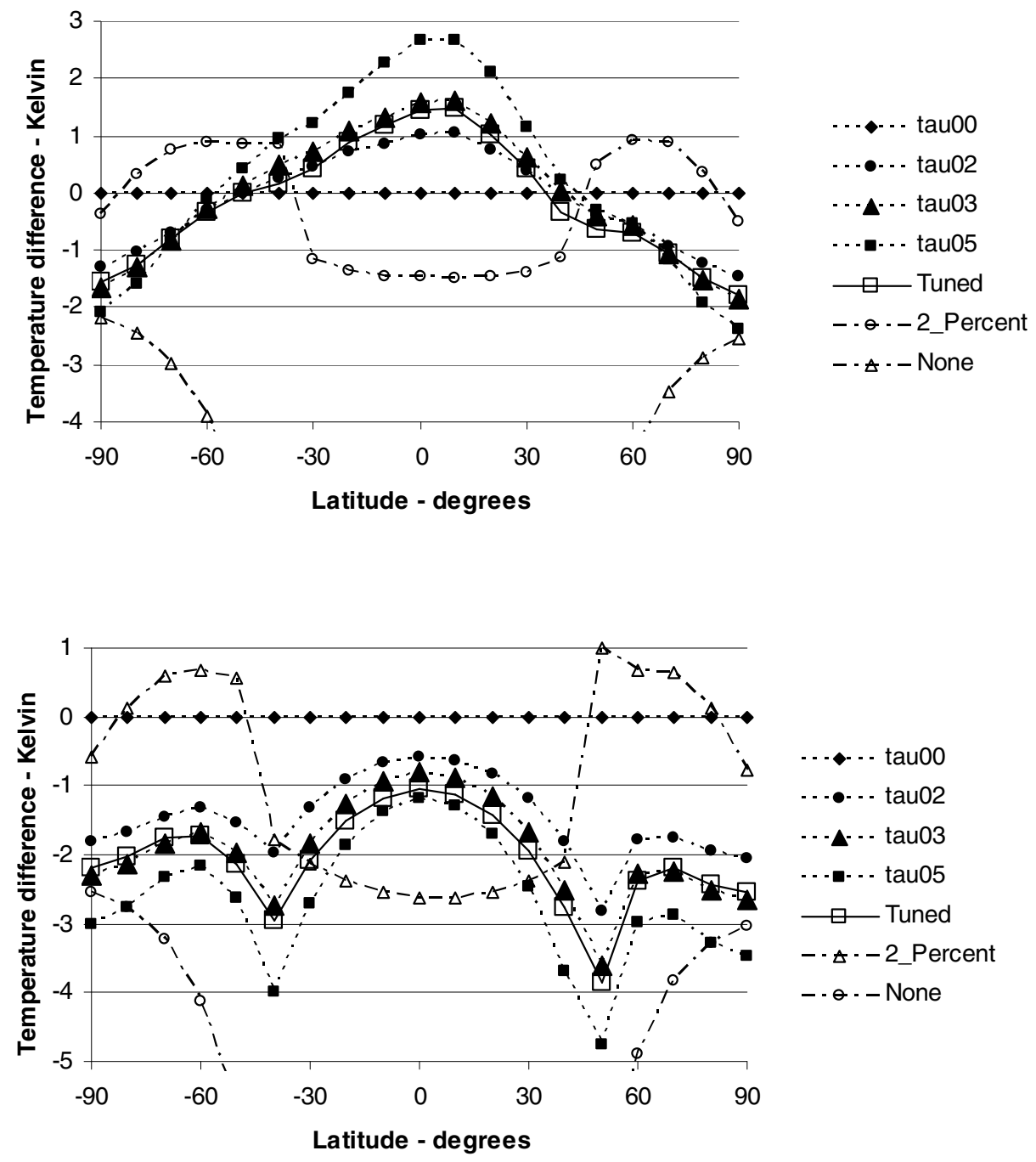

Figure 13 Average annual temperatures over latitudes with different atmospheric models for typical surface properties. The top panel shows results from bright/dusty ground (t.i. $=75 \mathrm{~S}$.I. units, albedo $=0.28$ ) and the bottom panel shows dark/rocky ground $($ t.i. $=220$ S.I. units, albedo $=0.13$ ). Tau* models refer to results of runs using a detailed atmospheric model with different dust opacities; $\tau=$ $0.0,0.2,0.3$, and 0.5 . Rather than show the range of absolute average temperatures (a range $>50 \mathrm{~K}$ ), shown here are the differences in average temperature of each atmospheric model with respect to the detailed atmospheric model with zero-dust opacity. The range of temperature differences is $\sim 5 \mathrm{~K}$ and this way is able to show the subtle difference between the models. Also shown are the average temperatures found when using the " $2 \%$ assumption," the "tuned" model developed in this project, and a model where the atmosphere has no effect on radiation ("none"). The tuned model was designed to closely follow the results of the $\tau=0.3$ model. Notice the effect of dust opacity on temperatures varies with latitude and surface properties. 
Figure 13 is also useful to demonstrate how dust is largely greenhouse neutral, as observed at Viking I landing site by Haberle and Jakosky (1991). The neutral effect of the dust was shown by both their model and illustrated by the global dust storm that produced almost no change in the average diurnal temperature at the Viking I Lander. The range of diurnal temperatures at the Lander was noticeably reduced by the dust storm, but the average temperatures did not change. Figure 13 suggests that the places where dust is truly greenhouse neutral is really a function of the surface properties and latitude. Dust tends to have a negative greenhouse effect at high latitudes over all surfaces. This can be explained by considering that the low angle of the illumination at high latitudes means atmospheric dust is more effective at scattering light back to space and increasing the planetary albedo (a cooling effect). Dust has a near neutral effect over surfaces with high albedo and high thermal inertia at low latitudes, such as the Viking I site, but has a positive greenhouse effect over high albedo, low thermal inertia surfaces at low latitudes.

Only a single atmospheric thickness was considered for most model runs in this project. That is to say we usually did not take into account the varying thickness of the atmosphere due either to elevation or seasons when calculating the temperatures of the surface and subsurface. In Section 2.3.1.1 we assess the effects of atmospheric thickness on the thermal models and find they are negligible. 


\subsubsection{Depletion of Water Vapor at Surface}

The idea that forms the basis of the water-vapor depletion scheme is that the part of the atmosphere that is in diffusive contact with ground ice, which we refer to as the "near-surface atmosphere," has a lower average content of water relative to the whole atmospheric column. The different components considered in the vapor depletion scheme (the ground, the near-surface atmosphere and the atmospheric column) are shown schematically in Figure 14. Also included in the figure is a summary processes between the components to deplete the near-surface atmosphere. Surface temperatures drop below the local water frost point regularly at night, even at relatively warm low latitudes, and thereby reduce the water content in the lowest part of the atmosphere by freezing it out. This depletion of water in the near-surface atmosphere can affect the depth to stable ground ice and the regional extent of stable ground ice. Evidence for depletion of water vapor in the near-surface atmosphere and frost formation has been implied or observed at martian lander sites such as Viking 1 and 2 and the Mars Exploration Rovers. 


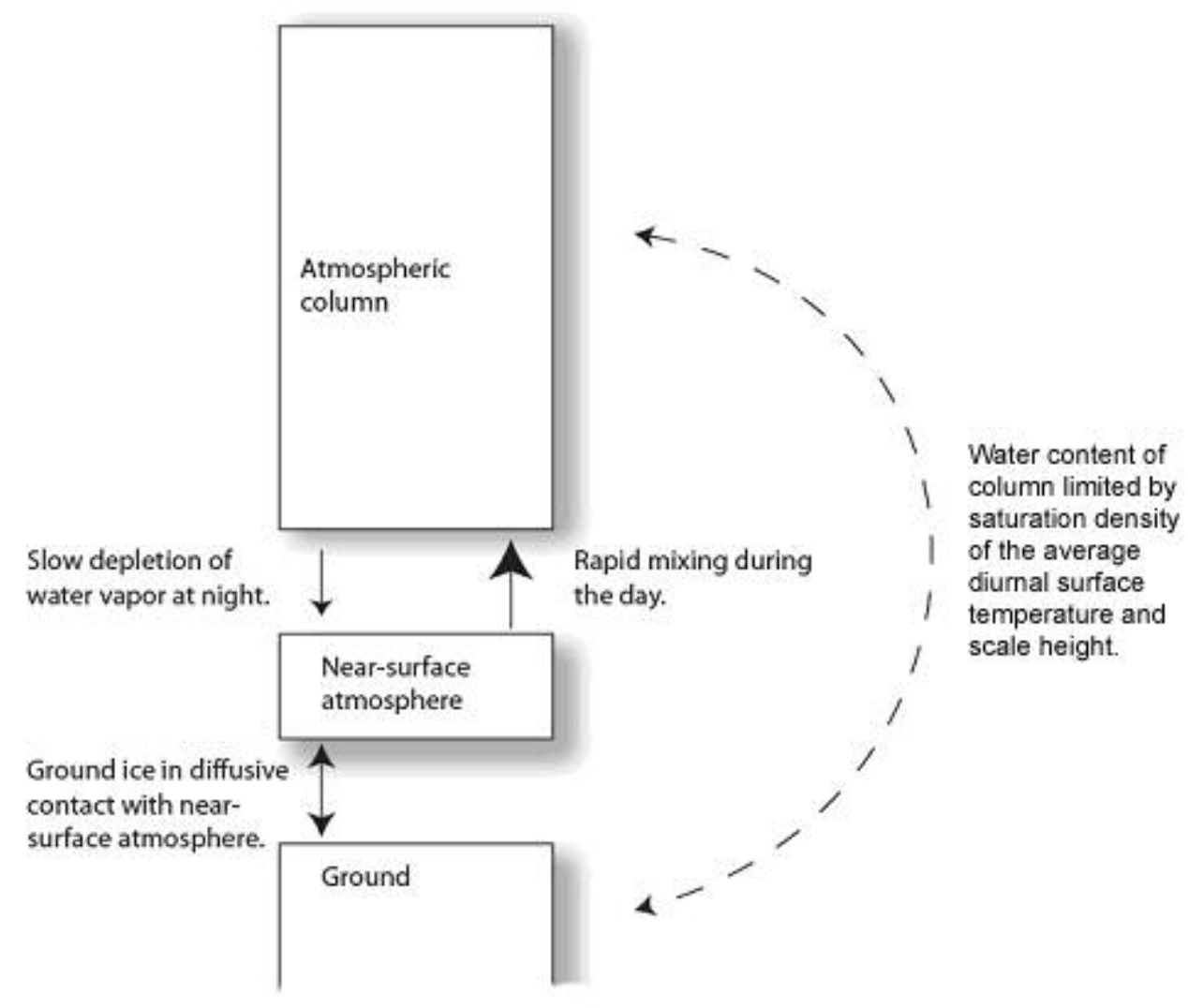

Figure 14 Cartoon showing the process of vapor depletion of the near-surface atmosphere. Frost on the surface depletes the near-surface of water vapor overnight with respect to the column. Rapid mixing by convection during day mixes water in this frost back into the atmosphere as it sublimes so the water content in the near-surface is assumed to be never greater than the equivalent density of the column abundance. 
Depletion of water vapor near the surface was inferred from apparent low water frost points observed at the Viking Lander sites (Ryan et al. 1982; Ryan and Sharman 1981). Temperatures recorded overnight by the landers showed a steady cooling trend with a small inflection at near the expected frost point for water vapor; the inflection was assumed to be due to the condensation. The water vapor densities were inferred from the temperature of the inflection. The vapor densities were found to be consistently less than the densities calculated from the water vapor column abundances found with instruments above the atmosphere and assuming the atmosphere is well mixed vertically. Note that there was no instrument onboard the Viking Landers to measure the atmospheric water content directly. This observed depletion was attributed to adsorption of water vapor onto the cooling ground surface (Jakosky et al. 1997) which depleted the amount of water in the near-surface atmosphere in the early evening while the temperatures were still above the frost point. Images around Opportunity Mars Exploration Rover have shown ephemeral frost in the early morning during the season when the northern pole is in exposed and there is more moisture in the atmosphere (NASA press release 2004). Temperatures are still too warm for this to be $\mathrm{CO}_{2}$ frost and this frost dissipates early in the morning. Presumably this frost has been derived from the atmosphere and reduced the near-surface water vapor density in the process.

Zent et al. (1993) presented a model that included surface-atmosphere interactions, including adsorption of water. In this model the calculated water mixing ratio shows water was depleted in the atmosphere from the surface up to a height of 
several 10s of meters during the night when the surface was cold. During the day this lowest layer of the atmosphere is rapidly mixed with the rest of the atmospheric column.

The depletion of water vapor we calculatein the near -surface atmosphere is a function of surface temperatures, so it can be calculated after running the thermal model for a particular latitude and set of properties of the ground. We describe how we calculate the depletion of water vapor in the following sections. In order for the model to calculate when surface temperatures are cold enough for frost to form and deplete the near-surface water on any particular day, the column abundance of water in the atmospheric must first be found as a function of season. We will first discuss how we calculate the seasonal water column. We will then demonstrate how the model calculates an effective water vapor density ratio for the near-surface atmosphere using the diurnal frost formed throughout the year. We will show some examples of the values of ratios calculated with different models. Later we demonstrate how sensitive the calculated ground-ice distribution is to the assumptions used in constructing the vapor depletion scheme. 


\subsubsection{Variation in Water Content of Atmospheric Column with Seasons}

We use a simple scheme to estimate the water cycle of the martian atmosphere which is used as a basis to find when the near-surface atmosphere is depleted by surface frost. Being 1-D, our model is not able to explicitly calculate the horizontal transport of water in the atmosphere. However, we do use the observation that the average measured water content of the atmospheric column over all seasons is largely independent of location and surface properties and design our own simple scheme to reproduce this observation. While the annual average water content of the atmosphere appears uniform over Mars, the water content of the atmosphere at any particular time certainly is not. The polar regions are extreme examples where during the winter the temperatures are too cold for the atmosphere to hold significant amounts of water vapor. However, in general, the water content of the atmosphere in high latitude regions is enhanced in the summers so that the annual average of the water content is the same as the average at low latitudes.

We assume that at any time there is some maximum amount of water vapor that can be held in the atmospheric column at which point it is saturated. We estimate this column abundance limit on any particular day $\left(\mathrm{col}_{\text {saturated }}\right)$ based on the ground temperatures with the equation,

$c o l_{\text {saturated }}=n_{\text {saturation }}\left(T_{\text {diurnal_average }}\right) \times H$,

where $n_{\text {saturation }}$ is the saturation vapor density which is a function of temperature, 
$T_{\text {diurnal_average }}$ is the average temperature of the surface the day for which the column abundance is being calculated, and $H$ is the scale height $(10500 \mathrm{~m})$. There are potential shortcomings with this approach. Among the processes that are not considered are the ice condensation level, changes in phase (i.e. cloud formation), diurnal variations in the water content and advection of heat and vapor; all of which may increase or decrease the amount of water vapor in the atmosphere. For this project though we limit ourselves to a simple approach and believe the average diurnal surface temperature should give a reasonable estimate of the maximum amount of water vapor in the atmosphere at any particular place and time.

After calculating the maximum column of water for each day of the martian year over a particular location, it is straight forward to calculate an average water column abundance at this location would have if the atmosphere were saturated throughout the year. We call the average of the daily maximum water columns the "water carrying capacity' of the atmosphere for a particular location and this will be a useful term later when we discuss estimates of atmospheric water content of Mars for other epochs.

The actual amount of water in the atmospheric column at any particular day and location is determined by either the maximum content based on the average diurnal temperature, or some arbitrary limit for that location, whichever is less. The column abundance of water on any day can then be written as, $c o l=\left\{\begin{array}{l}\text { limit }_{\text {tuned }}, \\ \text { col }_{\text {saturated }}\end{array}\right.$, whichever is less, where limit $_{\text {tuned }}$ is the arbitrary limit. This arbitrary limit is found for each combination of 
latitude and surface properties, so that the annual average water content for that location is equal to the uniform global content for the map being produced. The value of this limit is found using trial values of the arbitrary limit and testing if the average atmospheric water content derived is greater or less than the target content for the global water content of the epoch being tested. The final value of the arbitrary limit is found by bisection of successive trials. A similar approach to estimating the water cycle at any location was used in the vapor diffusion model of Mellon and Jakosky (1993).

In practice, this technique gives the atmospheric column abundance a constant value while the surface is warm during the local summer and a column abundance limited by the temperature of the location during the winter. Sample water cycles from this technique are shown in Figure 15. This figure shows the amount of water vapor in the column as a function of season with a limit applied so that the average annual water content matches a predetermined value, $15.7 \mathrm{pr}-\mu \mathrm{m}$ in this case, over dark, rocky ground over a range of latitudes. Figure 15 also shows the maximum amount of water vapor that can be held in the atmosphere. Note that the period of time that the water in the column is limited by the temperature of the location is longer for high latitudes where the winters are longer and colder. To compensate for the longer winters, the amount of water vapor allowed in the column over the summer is higher at high latitudes so that the annual average of the water content is the same. Note also that asymmetries in the water column, and surface temperatures, between the north and south are due to the eccentric orbit of Mars which places the planet furthest from the Sun as the north is in summer, cooling the planet slightly at this time. The amount of water in the atmosphere is 
consistent to first order with that observed, such as Figure 5 from Smith (2004). The timing of the periods of high water content at high latitudes from the model in Figure 15 are the same as the observed high water contents from Smith (2004). Also, the magnitude of the water content with the model is close (within a factor of 2) to the water contents observed. No attempt is made in Figure 15 to adjust the model water contents for elevation differences between different latitudes or differences in other surface properties. There are minor differences between the water cycle estimated by the model and the observed water cycle and we explore the potential impact this may have on water depletion scheme in the Section 2.2.3.3. 

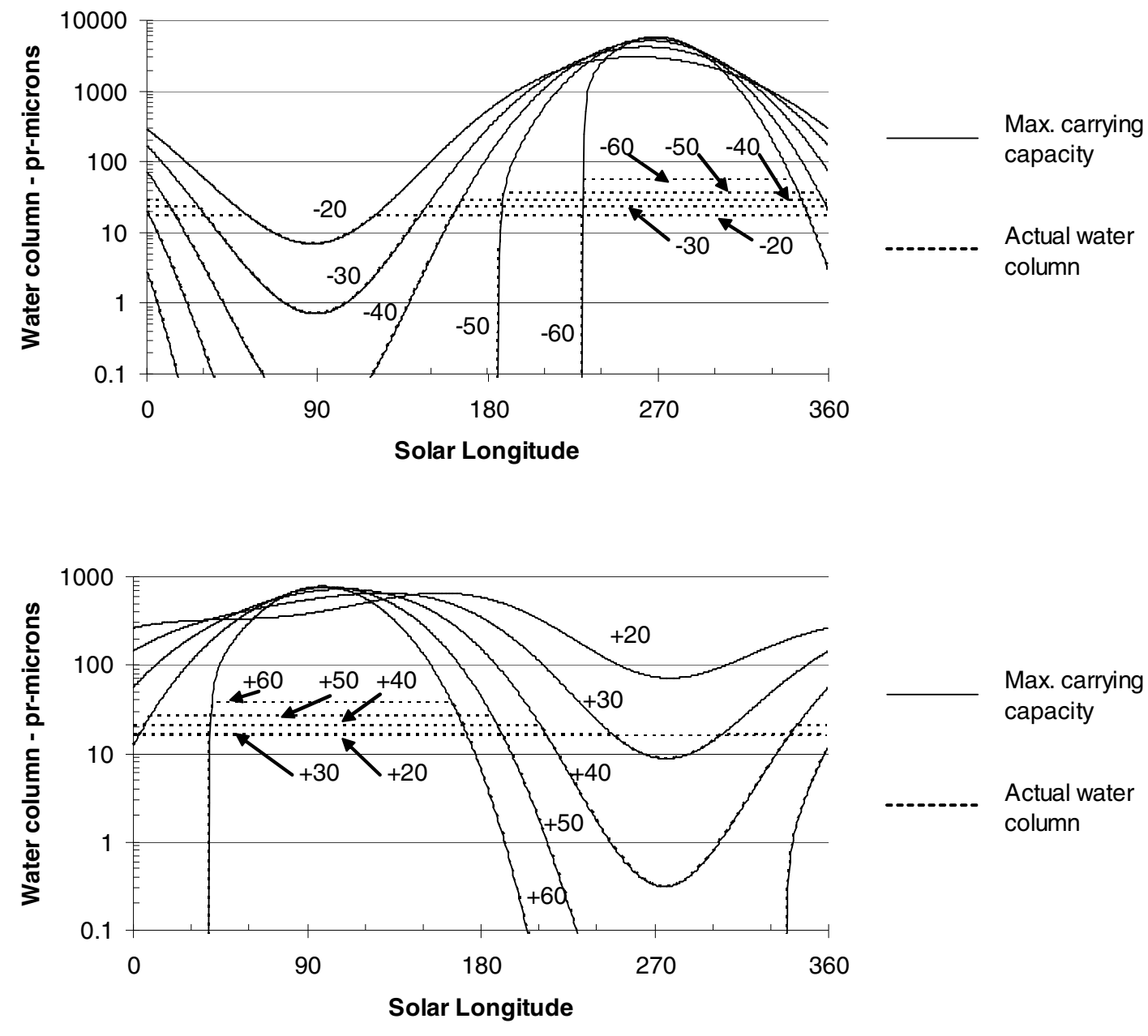

Figure 15 Estimated atmospheric water cycle over a dark, rocky surface at various latitudes. Shown are the maximum amount of water vapor that can be held in the atmosphere and the actual amount of water vapor in the column with a limit applied so that the average annual water content at each location matches a predetermined value of $15.7 \mathrm{pr}-\mu \mathrm{m}$ in this case. Asymmetries between the north and south are due to the eccentric orbit of Mars. For the model at $20^{\circ} \mathrm{N}$, the atmosphere has $15.7 \mathrm{pr}$ $\mu \mathrm{m}$ throughout the year. 


\subsubsection{Diurnal Depletion of Water Vapor in the Near-Surface}

For a particular location on Mars and on any particular day of the martian year, the scheme described above is used to determine the water content of the whole atmospheric column. This column abundance is used as a basis to determine the water vapor density of the near-surface atmosphere, the part of the atmosphere that would be in diffusive contact with ice in the ground, if present. The diurnal variation in the surface temperatures for that day are then used to determine if and how much the formation of surface frost will deplete the near-surface atmosphere of water vapor with respect to the atmospheric column.

When the surface is warm, the vapor density of the near-surface atmosphere is found by assuming that the atmosphere is well-mixed vertically. In this condition, the water vapor density can be found from the column abundance of water vapor. The equation to convert the water content of the atmospheric column to vapor density at the surface is

$n_{\text {equiv }}(\mathrm{col})=\frac{\operatorname{col} \times \rho_{\mathrm{H}_{2} \mathrm{O}}}{m_{\mathrm{H}_{2} \mathrm{O}} \times H}$,

where $n_{\text {equiv }}(\mathrm{col})$ is the vapor density in $\mathrm{H}_{2} \mathrm{O}$ molecules $\mathrm{m}^{-3}$, col is the column abundance of water in $\mathrm{m}, \rho_{\mathrm{H} 2 \mathrm{O}}$ is the density of water $\left(10^{3} \mathrm{~kg} \mathrm{~m}^{-3}\right), m_{\mathrm{H} 2 \mathrm{O}}$ is the mass of a water molecule $(2.99 \mathrm{e}-27 \mathrm{~kg})$ and $H$ is the atmospheric scale height $(10500 \mathrm{~m})$. 
Frost is assumed to form on the surface and reduce the water vapor density whenever the surface temperature drops below the frost point $\left(T_{\text {saturation }}\right)$ corresponding to the vapor density found by mixing with the atmospheric column. The water vapor density of the near-surface atmosphere ( $\left.n_{\text {near_suface }}\right)$ at any time is either the saturation vapor density determined by the surface temperature $\left(T_{\text {surf }}\right)$ at that time, or the equivalent density at the surface of the water content of the atmospheric column, whichever is less. $n_{\text {near_surface }}=\left\{\begin{array}{l}n_{\text {equiv }}(\mathrm{col}), T_{\text {surf }}>T_{\text {saturation }} \\ n_{\text {saturation }}\left(T_{\text {surf }}\right), T_{\text {surf }}<T_{\text {saturation }}\end{array}\right.$

In our scheme, frost on the surface is able to deplete the lowest part of the atmosphere during the cool night but it is not able to enhance the water vapor content during the warm day. In reality, there may be a brief period in the morning while frost sublimes and mixes back with the atmosphere that the water vapor density is enhanced at the surface. We assume this enhancement is minor with respect to the depletion overnight and this is consistent with the coupled ground-atmosphere models by Zent et al. (1993).

In order to find the average effective water vapor density of the near-surface atmosphere, the model first calculates the column abundance of water vapor for every day of the martian year. Then the water vapor density is found for the near-surface atmosphere for every time step of each day. The vapor density is averaged over all time steps in the martian year. A ratio is found of the vapor density of the near-surface atmosphere found by the model and what it would have been without depletion, this is the 'effective water vapor density ratio.' The difference between 1 and this ratio is the amount of water vapor depletion due to frost formation in the model for a particular 
location. This effective ratio is useful because it is convenient value that is relatively slowly varying with respect to other properties and can be interpolated.

The effective vapor ratio is calculated as the thermal model is running to find temperatures and ice-table vapor densities as functions of ice depth. The amount of water in the atmosphere is generally considered a function of elevation, which varies with location. The effective vapor ratio of the near-surface atmosphere is determined for a range of water contents in the atmosphere and the ratio is interpolated to the actual water content for a particular location (or elevation) for a particular map of stable ground ice as it is being produced.

The average annual vapor density of the near-surface atmosphere can now be related to the average global column abundance of water vapor in atmosphere. The equation is in a similar form to the equation to find the equivalent density of vapor at the surface as a function of column abundance. The average vapor density of the nearsurface can now be written as,

$$
\begin{aligned}
n_{\text {effective }} & =R_{\text {effective }} \times n_{\text {equiv }}(\mathrm{col}) \\
& =R_{\text {effective }} \times \frac{\operatorname{col} \times \rho_{\mathrm{H}_{2} \mathrm{O}}}{m_{\mathrm{H}_{2} \mathrm{O}} \times \mathrm{H}},
\end{aligned}
$$

where $R_{\text {effective }}$ is the effective vapor density ratio and col is now the average column abundance of water vapor in the atmosphere, and the other symbols are as they were used before.

For an example, one of the model latitudes in Figure 15 is shown again in Figure 16 which also shows the average vapor density of the near-surface atmosphere each day as a function of season. Figure 17 shows vapor density cycles of the near-surface 
atmosphere through the day for selected days within the year. Within the diurnal cycle, the near-surface vapor density is determined by the either the surface temperature or the column abundance. Shown on Figure 17 is the saturation vapor density of water ice determined by the surface temperature; this is the maximum vapor density that could be found at any particular time. During warm day temperatures, the saturated vapor density is greater than the vapor density derived from the column abundance so it is the column that determines the near-surface atmospheric vapor density during the day. During the cool night, it is the saturated vapor density of the surface temperature that determines the near-surface atmospheric vapor density. Note that during the local summer, the nearsurface vapor density is a function of the column abundance for a larger fraction of the day, meaning that there is less depletion in the summer than in the winter. In the winter the near-surface vapor density is limited by the surface temperature for most of the day. 


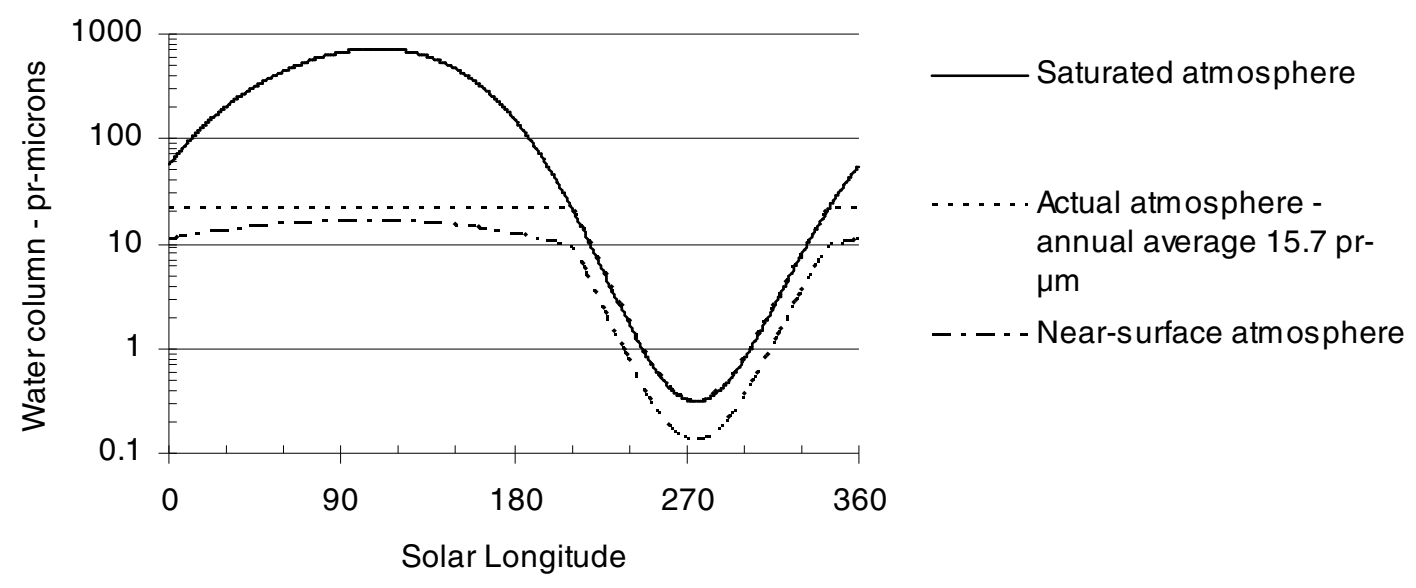

Figure 16 The amount of the water in the atmosphere over dark, rocky ground at $40^{\circ} \mathrm{N}$. This matches one of the latitudes shown in Figure 15. Like Figure 15, the maximum amount of water vapor the atmospheric column can hold and the actual amount of water are shown. Also shown is the average amount of water vapor in the near-surface atmosphere which is always less than the amount of the water in the column due to the formation of frost overnight. The degree of depletion is greater in some seasons than others, as shown in examples in Figure 17. The vapor density of the near surface is converted to equivalent $\mathrm{pr}-\mu \mathrm{m}$.


Figure 17 Vapor depletion within the diurnal cycle. The model used to generate these panels is the same as the model used for Figure $16 ; 4^{\circ} \mathrm{N}$ with dark and rocky properties. Water vapor densities are converted to column abundances in pr- $\mu \mathrm{m}$. The solid line shows the saturation water content at any particular time. The dotted line represents the actual vapor density of the near-surface atmosphere that is equal to the water content of the column, as read from Figure 16, or a saturation limit determined by the temperature of the surface. In these plots, the near-surface is depleted when the actual water content is limited by the saturated content. The water content is shown for two Sols, one in summer $\left(\mathrm{L}_{\mathrm{s}}=\mathbf{1 0 0}^{\circ}\right)$ when atmosphere is capable holding a lot of water vapor and the nearsurface atmosphere is only depleted for a short period in the coldest part of the night. In contrast, the near-surface atmosphere in the winter $\left(\mathrm{L}_{\mathrm{s}}=250^{\circ}\right)$ is depleted for most of the diurnal cycle. Note also the different value ranges on the $y$ axis. 
The values that are found for the water carrying capacity, the arbitrary limit tuned to match the global content, and the effective vapor density ratio are all functions of the surface properties. For the example shown in Figure 16 and Figure 17, a model at 40 N, thermal inertia of 220 S.I. units and an albedo of 0.13 , the atmosphere would have a maximum water content, or a water carrying capacity of $254 \mathrm{pr}-\mu \mathrm{m}$ (equivalent to a vapor density of $8.1 \mathrm{e} 20$ molecules $\mathrm{m}^{-3}$ at the surface) if it were saturated every day of the year based on the average diurnal surface temperatures, as described above. In order for the atmosphere to hold $15.7 \mathrm{pr}-\mu \mathrm{m}\left(5.0 \mathrm{e} 19\right.$ molecules $\left.\mathrm{m}^{-3}\right)$ when averaged over all seasons, the atmospheric column would be saturated or contain a arbitrary limit equivalent to a density of $21.2 \mathrm{pr}-\mu \mathrm{m}$ (6.8e19 molecules $\mathrm{m}^{-3}$ at the surface) each day of the year with the scheme we employ. In this case, the average vapor density of the near-surface atmosphere, in contact with ground ice, would only be $63 \%\left(3.18 \mathrm{e} 19\right.$ molecules $\left.\mathrm{m}^{-3}\right)$ of what it would be without depletion due to surface frost. Other values for a model with a thermal inertia of 75 S.I. units, an albedo of 0.28 and latitude of $40^{\circ} \mathrm{N}$ are shown in Table 3. Note that the effective vapor ratio is lower for the dusty surface; ground with lower thermal inertia has a larger diurnal temperature cycle which enhances nighttime frost depletion and decreases the effective vapor ratio. Likewise, low latitudes with stronger diurnal cycles typically experience more vapor depletion than seen in Table 3. 


\begin{tabular}{|c|c|c|c|}
\hline Model & & $\begin{array}{c}\text { Dark and } \\
\text { Rocky }\end{array}$ & $\begin{array}{c}\text { Bright and } \\
\text { Dusty }\end{array}$ \\
\hline Latitude & degrees & 40 & 40 \\
\hline Thermal inertia & S.I. units & 220 & 75 \\
\hline Albedo & & 0.13 & 0.28 \\
\hline $\begin{array}{l}\text { Max. atmospheric water } \\
\text { carrying capacity }\end{array}$ & $\mathrm{pr}-\mu \mathrm{m}$ & 253.9 & 40.0 \\
\hline $\begin{array}{l}\text { Water limit tuned to produce } \\
\text { average of } 15.7\left(5 \mathrm{e} 19 \mathrm{~m}^{-3}\right)\end{array}$ & pr- $\mu \mathrm{m}$ & 21.2 & 29.0 \\
\hline $\begin{array}{l}\text { Atmospheric frost } \\
\text { temperature }\end{array}$ & Kelvin & 200.9 & 203.1 \\
\hline $\begin{array}{l}\text { Water vapor density of near- } \\
\text { surface atmosphere }\end{array}$ & $\mathrm{m}^{-3}$ & $3.18 \mathrm{e} 19$ & $2.48 \mathrm{e} 19$ \\
\hline $\begin{array}{l}\text { Ratio of effective near surface } \\
\text { atmosphere density }\end{array}$ & & $63.6 \%$ & $49.6 \%$ \\
\hline
\end{tabular}

Table 3 Sample results of the water vapor depletion algorithm. Results are shown for dark/rocky and bright/dusty surfaces at a mid-northern latitude. The higher average surface temperature of the dark/rocky ground increases the amount of water that the atmosphere above it can hold. The smaller variation in diurnal surface temperatures of the rocky model decreases the depletion of water vapor at the surface so the effective vapor density at the surface is higher.

Figure 18 demonstrates the variation in the effective water vapor density ratio of the near-surface atmosphere as a function of ice depth and atmospheric water content. The effective ratio values are calculated using the surface temperatures from the thermal model as described in the sections above. In general the ratio decreases when ice is deeper and when there is more water in the atmosphere. However, there is no simple function for the effective ratio; at high water contents there is a small increase in the ratio as the depth to ice increases while ice is well below the diurnal wave but still within the seasonal wave. This small increase is related to higher surface temperatures in certain days of the summer season as ice becomes deeper. 


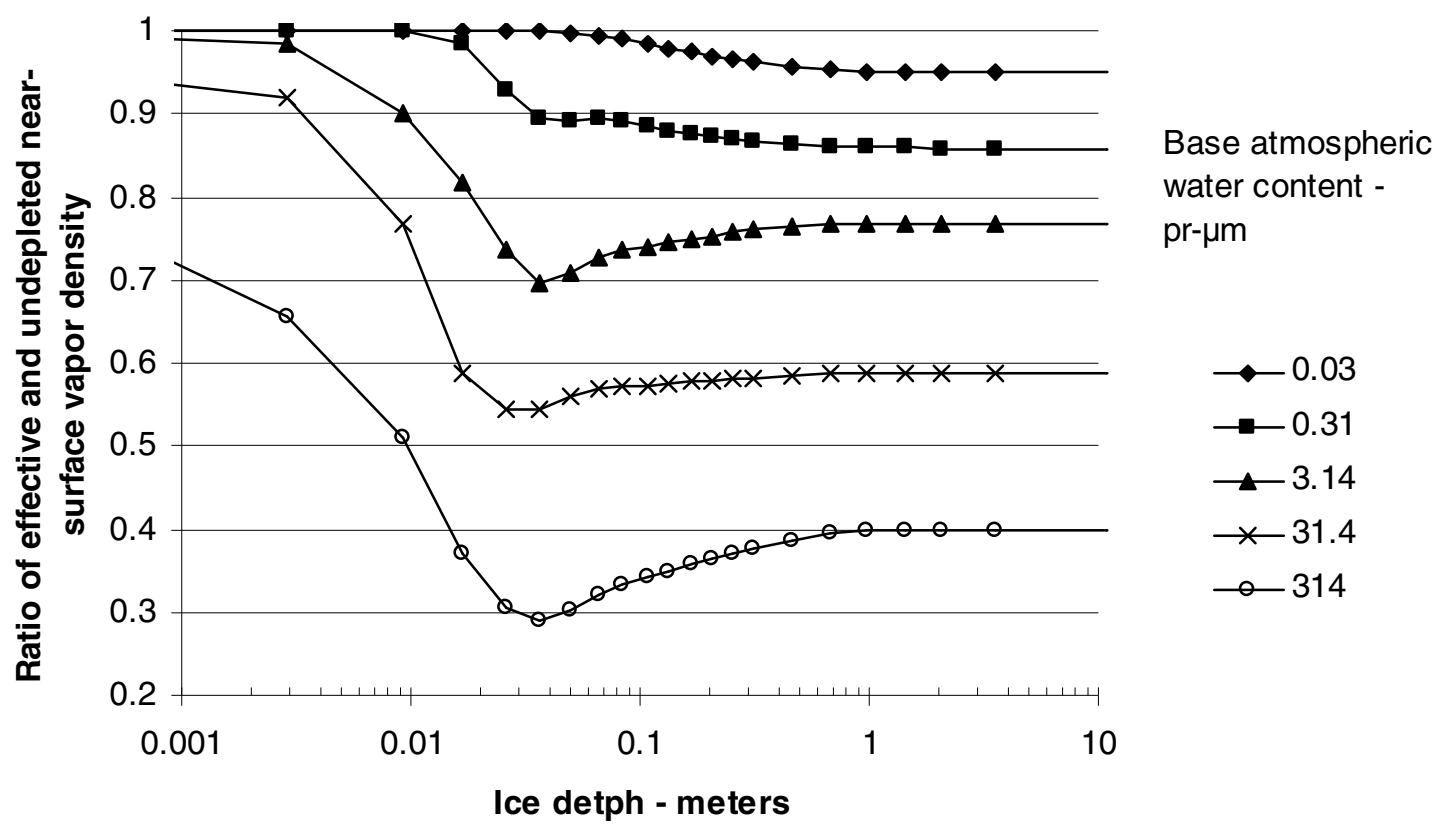

Figure 18 The effective water vapor density ratio of the near-surface atmosphere, which is with respect to the average density if no depletion took place. The thermal model used to generate these results used a latitude of $40^{\circ} \mathrm{N}$, a thermal inertia of 220 S.I. units and an albedo of 0.13 . The ratio decreases with ice depth for shallow depths less than the diurnal skin depth because shallow ice dampens the diurnal thermal wave and the associated vapor depletion. The effective ratio also decreases with increasing base atmospheric water contents. The vapor depletion scheme and effective ratio ensure that the atmospheric column and near-surface atmosphere are never supersaturated with water vapor. 
A water-vapor depletion has been used before in calculating the distribution of martian ground ice in the present epoch (Mellon et al. 2004). Near the mid latitudes and near the present boundary of ground ice, the depletion factor used by Mellon et al. was about 0.6. This happens to be similar to the factor we find with our depletion at these latitudes as well. However this is the only similarity between the two depletion schemes. Our scheme is based on the diurnal cycle, making depletion more effective at low latitudes where the diurnal cycle is strongest. At high latitudes there is little depletion because the diurnal cycle is weaker relative to the seasonal cycle. The depletion factor used by Mellon et al. does the exact reverse, depleting water vapor at high latitudes. This lower water vapor density near the poles makes ice deeper in their model, though the temperatures are still cold enough that ice is close to the surface and the difference is hard to notice when looking at maps of ice depth. So while the two depletion schemes produce similar results in the present epoch, they are in fact completely different in how they work and would have very different results in different epochs.

We do not attempt to account for adsorption here as done by Jakosky et al. (1997). The effect of adsorption is a function of surface composition, grain size and temperature. Frost formation is relatively simple and only a function of temperature. Including adsorbed water could enhance depletion further as it can deplete water vapor on the cooling surface at temperatures still greater than the frost point. 


\subsubsection{Sensitivity to vapor depletion}

There are many assumptions that go into the part of the model that estimates the ratio of average content of water vapor of the near-surface atmosphere relative to the water content of the whole column. Many of the assumptions are necessary in order to estimate the content of water in the atmospheric column with a 1-D model. In reality the water content is a function of interactions with the surface as well as atmospheric circulation which is not included explicitly in our model. We can, however, test the sensitivity of the estimated effective vapor density ratios to variations in the assumptions that we used. Here we test the way we estimate how much water vapor can be held in the atmospheric column on any particular day. As described before, typically we assume that the column water content is either limited by the temperature of the column that day, or it is some arbitrary limit that is determined so that the column water content is some fixed value when averaged over all seasons - presumably determined in part by atmospheric circulation. While we argue that this simple approach gives a reasonable reproduction of the water cycle that has been observed in the martian atmosphere (e.g. Smith 2002), there are processes that may in some seasons put more or less water vapor in the atmospheric column than our simple estimate. As a way to consider the effect this may have, we now include an offset term to the average diurnal surface temperature that is used to find the column water content when it is saturated. Now the water column on any particular day can be expressed as, 
$c o l=\left\{\begin{array}{l}\text { limit }_{\text {tuned }}, \\ \operatorname{col}_{\text {saturated }}=n_{\text {saturation }}\left(T_{\text {diurnal_average }_{\text {a }}}+\text { offset }\right) \times H\end{array}\right.$, whichever is less.

When the offset is positive, this increases the amount of water that can be held in the column. The limit $_{\text {tuned }}$ is found in the same way as used before, that is, so that the average of col over all seasons is some fixed value. An example of the change in the estimated water cycle is shown in Figure 19. The offsets applied in this Figure are $+5 \mathrm{~K}$ and $-5 \mathrm{~K}$, which increase and decrease the saturation limit to the water carrying capacity of the atmosphere respectively. For the example shown, there is very little variation in the arbitrary limit used in order that the annual average for the water column abundance to be $15.7 \mathrm{pr}-\mu \mathrm{m}$

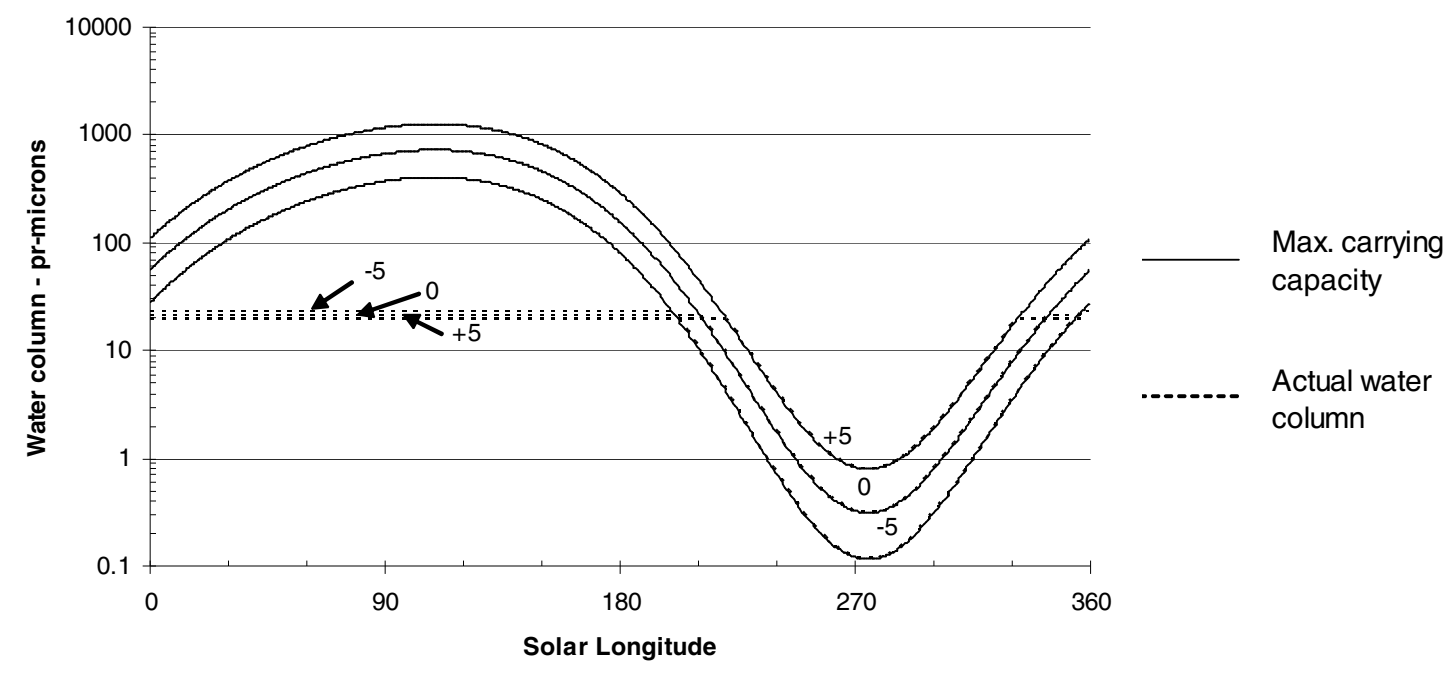

Figure 19 Change in water cycle for a model at $40^{\circ} \mathrm{N}$ and dark, rocky surface properties (thermal inertia $220 \mathrm{~S}$. I. units and albedo 0.13). Water cycles are shown for different offset values as described in the text. Offset values are labeled on the figure and used in relating the saturated water carrying capacity of the atmosphere to the surface temperature. The actual water column for each model run is tuned so the annual average is $15.7 \mathrm{pr}-\mu \mathrm{m}$. 
Using the water column found with an offset applied, we then calculate the effective ratio of the annual average vapor density of the near-surface atmosphere in the same way as described in Section 2.2.3.2. The near-surface vapor density at any time step is either determined by the water column of that particular day, or the saturation vapor density determined by the surface temperature at that time step and effectively depleting the near-surface atmosphere. The effective ratio is then the average of the vapor density of the near-surface atmosphere including depletion due to frost for every time step over the full year, relative to the average vapor density if there were no depletion. The calculated effective vapor density ratios over dark, rocky ground with no ground ice are plotted as a function of latitude in Figure 20. There are only minor changes in the value of the ratio, $<10 \%$, for the offsets used in calculating the water content of the column. Note that temperature offsets of $5 \mathrm{~K}$, used in a way described above, change the amount of water that can be held in the atmosphere by about a factor of 2. 


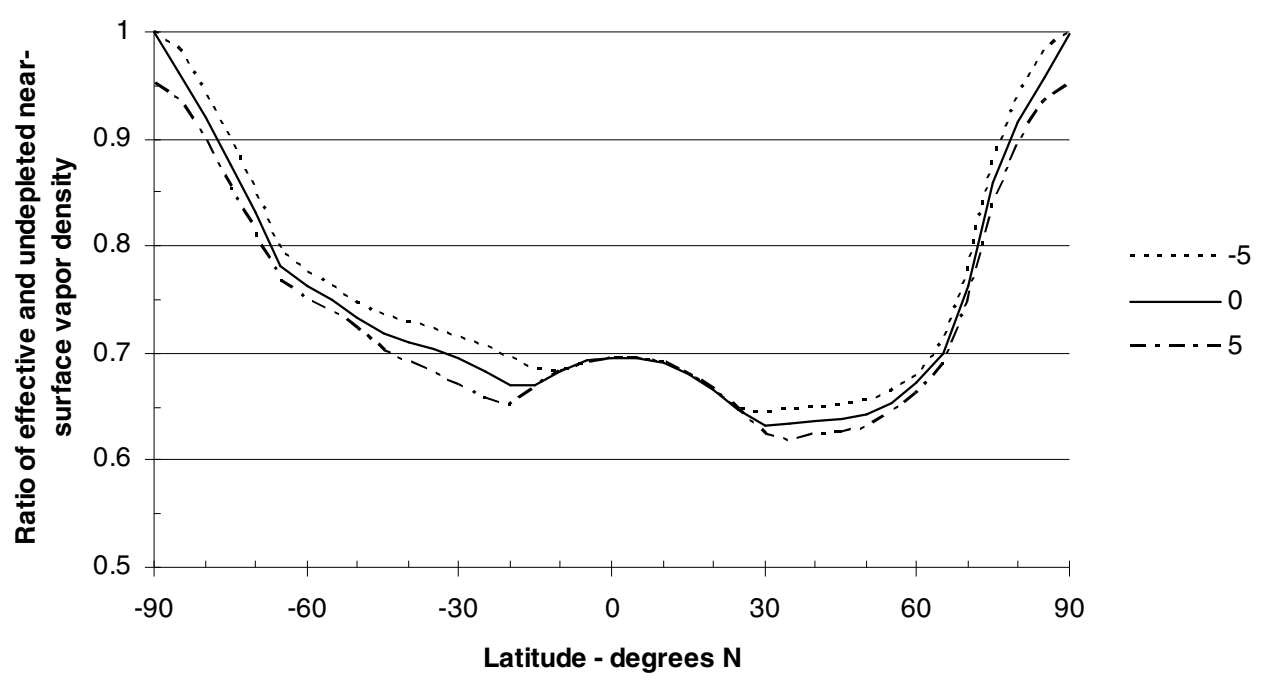

Figure 20 Effective ratios of annual average vapor density of the near-surface atmosphere over dark, rocky surface with no ground ice as a function of latitude when the average annual water column is $15.7 \mathrm{pr}-\mu \mathrm{m}$.

The effective vapor density ratio for the near-surface atmosphere may also be sensitive to processes that affect the vertical distribution of water in the atmospheric column. Typically, we calculate an equivalent vapor density in the near-surface atmosphere from the column abundance of water vapor with the assumption that the atmosphere mixed uniformly vertically. However, there could be a vertical mixing processes that act over the diurnal or seasonal cycle that produce either a systematic enhancement or depletion of the water content of the near-surface atmosphere relative to the atmospheric column. This enhancement or depletion would be in addition to the depletion by frost at night. The effects of any vertical systematic enhancement or depletion would be the same as applying different water contents to the whole atmosphere and assuming vertical mixing is uniform. Instead of running specific models 
to test the sensitivity of the distribution of stable ground ice to systematic enhancements/depletions, we can instead test the sensitivity of stable ice to changes in the amount of water in the atmosphere while still assuming vertical mixing. This test will be done later.

\subsubsection{Determining Stable Depth of Ice}

In order to calculate the depth at which ground ice is stable, a two-layer version of the thermal model described in Section 2.2.2 is used. Ice is assumed to be present in the ground starting at some depth and continuing to the base of the thermal model. The ice gives the ground high thermal inertia properties where it is present. Above this depth, or ice table, the ground is ice free and the thermal properties are those that are determined with remote sensing. The thermal model is run for a series of assumed depths to the ice table and the average vapor density at the top of the ice is calculated. Ground ice is considered stable when the average water vapor density above the ice is equal to the average vapor density in the atmosphere just above the surface, as discussed in Section 2.2.1.

The most significant change in properties of the ice-rich ground is the thermal conductivity which is orders of magnitude greater than the thermal conductivity of the top dry layer. The properties of the ice-rich ground here are assumed to be: thermal conductivity $(k) 2.5 \mathrm{~W} \mathrm{~m}^{-1} \mathrm{~K}^{-1}$, density $(\rho) 2000 \mathrm{~kg} \mathrm{~m}^{-3}$ and a specific heat $(c) 1000 \mathrm{~J} \mathrm{~kg}^{-1}$ 
$\mathrm{K}^{-1}$, which give a thermal inertia of 2236 S.I. units $($ t.i. $=\sqrt{k \rho c})$. The thermal property values for what we call the ice-rich layer do not change much if this layer of the thermal model is composed of solid water ice, a soil-ice mix or even solid rock. We do not consider the values critical to the results shown in this paper; what is significant is that the thermal inertia of this layer is so much greater than the top layer. Modifying the thermal property values of the ice-rich layer will only modify the depth to stable ice; it will not modify the regional extent of stable ice. Ice is deepest when the stability of ground ice is most marginal, i.e. the equatorward limit of the ground ice deposits. When ice is deepest the ice has the least effect on the temperatures at the surface, hence the properties of the ice-rich layer will not affect the limit of the stable ice deposits.

The top of the ground ice is typically defined at the mid-point of one of the layers within the model. This way the thermal conductivity between mid-points is uniform. The density and specific heat properties of this layer are adjusted for the combination of ice-free and ice-rich compositions. An alternative is to define the top of the ice-rich ground at a layer boundary, then layer properties are uniform and effective thermal conductivity between mid-points is calculated for a combination of materials in series across the top of the ground ice. Both approaches were tried and were found to give identical trends in vapor density over ice as functions of depth.

In order to calculate the depth to stable ice, the thermal model is run for a series of assumed ice depths. In multiples of the diurnal skin depth, the depths tested by the models are: $0,0.1,0.32,0.584,1.28,2.28,4.68,10.8,34.1,125,375$, and a model with no ice. Note that the seasonal thermal skin depth on Mars is 26 times deeper than the 
diurnal skin depth. Usually, if ground ice is stable it is stable at a depth less than the seasonal skin depth, though the model tests beyond this seasonal skin depth to catch the few cases where ice is stable deeper. The model calculates the water vapor density at the top of the ground ice for each time step in a full martian year and finds the average icetable vapor density; an example is shown in Figure 21. The equation used to find the vapor density over water ice as a function of temperature at each time step is the same that was used by Mellon and Jakosky (1993)

$n_{\text {sat }}=\frac{4.7 \mathrm{e} 26}{T} \exp \left(\frac{-5.7138 \mathrm{e} 3}{T}+3.6849 \times \ln T-(7.7532 \mathrm{e}-3) \times T\right)$,

where $n_{\text {sat }}$ is the vapor density in molecules $\mathrm{m}^{-3}$, and $T$ is the temperature in Kelvin. This form of the equation includes the effect of the difference in specific heat of ice and vapor as functions of temperature on the latent heat of sublimation. The equation can also be well represented using a simpler version of the Clausis-Clapyeron equation assuming a constant latent heat. Figure 21 also shows examples of thermal model results as functions of ice depth. Shown are the changes in average annual surface temperature, the ice-table vapor density, maximum $\mathrm{CO}_{2}$ frost build up and the number of days seasonal frost is present for ground at a latitude of $70^{\circ} \mathrm{N}$, an albedo of 0.2 and a thermal inertia of 300 S.I. units $\left(\mathrm{J} \mathrm{m}^{-2} \mathrm{~K}^{-1} \mathrm{~s}^{-1 / 2}\right)$. 



Figure 21 Variations in surface model results as a function of ice depth. The latitude of the model shown is $70^{\circ} \mathrm{N}$, thermal inertia is $300 \mathrm{~S}$.I. units $\left(\mathrm{J} \mathrm{m}^{-2} \mathrm{~K}^{-1} \mathrm{~s}^{-1 / 2}\right)$, and albedo 0.20 . The general trend is that the average surface temperature decreases as ice becomes deeper in a similar way that average temperatures drop as thermal inertia decreases. The diurnal thermal wave of the top layer is $3.5 \mathrm{~cm}$ and the seasonal wave is $91 \mathrm{~cm}$. Ice has little effect on results once it is deeper than a seasonal skin depth. It is when this vapor density is equal, or less than, the average near-surface atmospheric vapor density that ground ice is considered stable. Decreasing surface temperatures with ice depth coincide with increasing amounts of seasonal $\mathrm{CO}_{2}$ build up and length of time seasonal frost is on the ground; both of which are significant for atmospheric circulation models. 
The ice-table vapor density is highest when ground ice is closest to the surface; this is because vapor density is primarily an exponential function of temperature and the average in vapor density will be higher at places that experience higher temperatures in the diurnal and seasonal cycle, i.e. close to the surface. Ice-table vapor density decreases as ice becomes deeper and ice is considered stable if this ice-table vapor density is equal to the average water vapor density of the local near-surface atmosphere; an example is shown in Figure 22. Depletion of water vapor in the near-surface atmosphere is taken into account in this figure. Note that the effective vapor ratio used is a function of the surface temperature cycle, so it is also a function of ice depth. The difference between the ice-table vapor density and the effective vapor density of the near-surface atmosphere is found for each ice depth. These differences in vapor density are then interpolated as a cubic spline of the log of the ice depth to determine the ice depth that corresponds to a zero difference in vapor densities. This depth is then the stable ice depth for the model. Ground ice is unstable and will be in the process of subliming whenever the ice table is at a depth such that the ice-table vapor density is greater than effective vapor density of the near-surface atmosphere. Conversely, water vapor will condense onto the ice table - in the absence of other processes - if the ice-table vapor density is less than the density in the atmosphere.

The depth to stable ground ice in the example shown in Figure 22 is $4.72 \mathrm{~cm}$. The interpolations shown in Figure 22 are simple linear interpolations shown as a guide. Cubic spline interpolations are still used to estimate the stable ice depth quoted. Also shown on Figure 22 are effective vapor densities of the near-surface atmosphere where 
the way that the saturation limit for the water vapor column abundance is allowed to vary by applying an offset to the average diurnal surface temperature, see Section 2.2.3.3 for details on this calculation. The effective vapor densities of the near-surface atmosphere is shown for offsets of -5 and $+5 \mathrm{~K}$, and as indicated in the Figure 22 the is a negligible change in the depth to stable ice, i.e. where the vapor density is equal to the ice-table vapor density. Using cubic splines, the depths to stable ice become 4.64 and $4.82 \mathrm{~cm}$ for offsets of -5 and $+5 \mathrm{~K}$ respectively.

The underlying assumption is that the ground ice is in diffusive contact with the atmosphere. This is the same method used by, for example, Mellon et al. (2004) and Schorghofer and Aharonson (2005), see the discussion in Section 2.2.1. As discussed by Mellon, the effect of including the high thermal conductivity of the ground ice is to bring the ground ice much closer to the surface due to the attenuation of the seasonal thermal wave at depth by the high thermal conductive layer. 


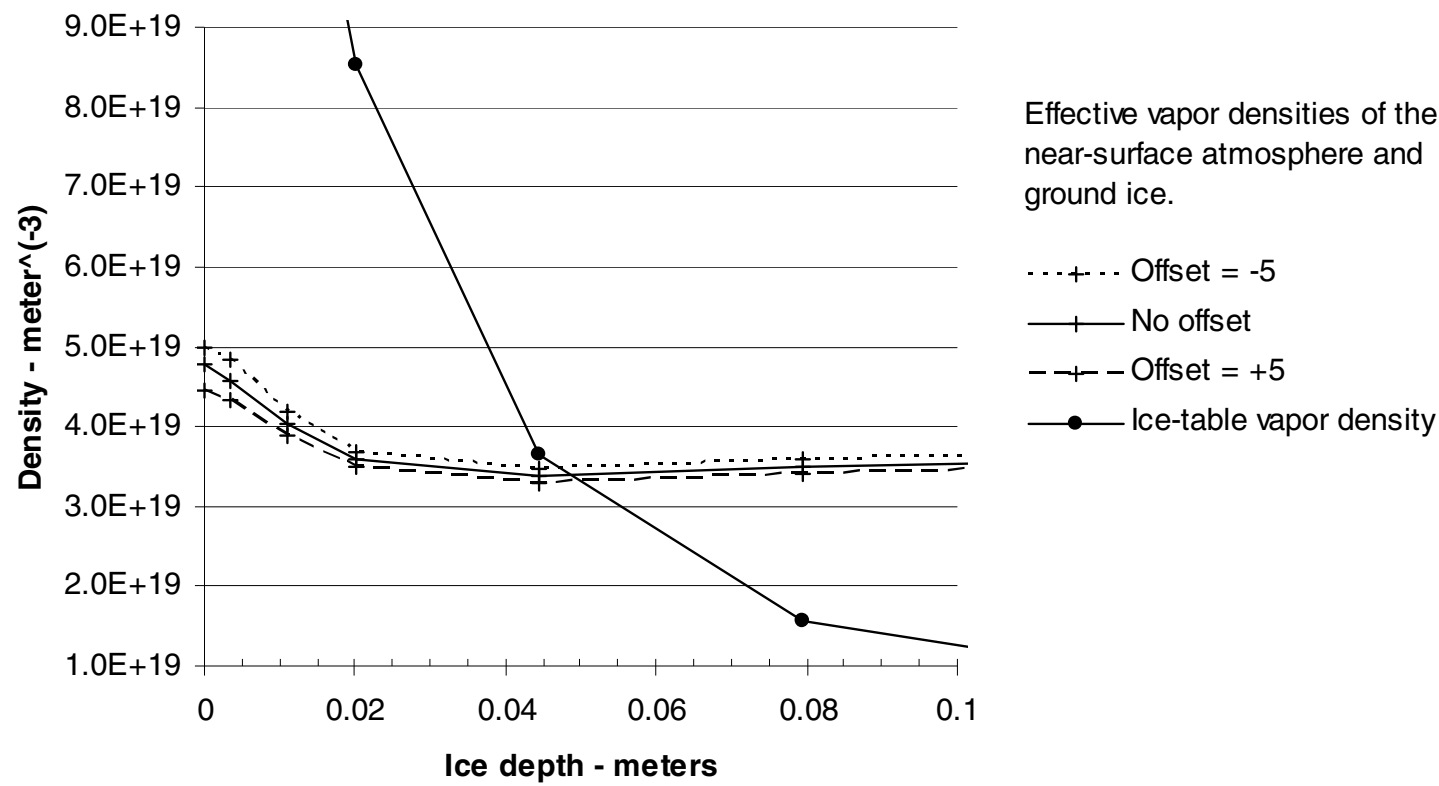

Figure 22 Determining depth to stable ice. The stable ice depth is where the ice-table and nearsurface atmosphere vapor densities are equal. The ice-table vapor density as a function of depth is the same as shown in Figure 21, though it is shown here on a linear-linear plot to show the region of interest. The vapor density of the near-surface atmosphere is also shown where temperature offsets shown are values applied in the vapor depletion scheme, see text for details. The modifications to the vapor depletion model have only very small effects on the depth to stable ground ice. The average water content of the atmospheric column used for the data shown is $15.7 \mathrm{pr}-\mu \mathrm{m}$ (equivalent to a density of $5 \mathrm{e} 19$ molecules $\mathrm{m}^{-3}$ at the surface). 


\subsubsection{Distribution of Atmospheric Water}

For most of the maps of stable ground ice we will show here, we assume there is a single value for the seasonally averaged water vapor column abundance in the atmosphere across all of Mars. The only correction that is routinely made is for the elevation, as done with the equation below

$n(z)=n_{0} \exp \left(\frac{-z}{H}\right)$

where $n(z)$ is the average vapor density of water as a function of height, $z$, above a base level, $n_{0}$ is the equivalent vapor density of the global average water vapor column abundance, and $H$ is the scale height of the atmosphere $(10500 \mathrm{~m})$. This correction has the effect of increasing the water vapor density at locations with low elevations simply because the atmosphere is thicker there.

The assumption that the atmospheric water content is uniform is necessary in a sense for our maps of ground ice at other epochs. In the absence of any observations/data of atmospheric water content from past epochs or any climate models with the required detail from these times, the assumption of a uniform distribution is the only realistic approach. Indeed, this assumption has been used before even for the present epoch (Mellon and Jakosky 1993; Mellon et al. 2004).

The average global water vapor column abundance of the martian atmosphere does appear to be very close to uniform based on observations from TES (Smith 2002), as 
discussed in Section 2.1.2. Close examination of the average water content shows there are still variations of the order of a factor of 2 regionally. As argued before, it is interesting there is not greater variation seen given to wide range of seasonal atmospheric water contents. We argue here that while the assumption of a uniform water content of the atmosphere is unlikely to be exactly true at any epoch, it is still a good representation of water content for our purposes and that even a variation of a factor of a few will not significantly change the ground-ice distributions that we will show.

\subsubsection{Generating Maps of Ground Ice Distribution}

In order to generate maps of ground-ice distribution at any epoch, temperatures and ice-table vapor densities must be found with the thermal model for a wide range of conditions to cover the globe of Mars. Then we find the average water vapor density of the near-surface atmosphere that is in diffusive contact with the subsurface to find where ice is stable and how deep it will be. The most computational intensive part of this process is the thermal model. Rather than run the thermal model at every single coordinate shown on our maps, a look-up table is generated and results are interpolated to each map location and property values of the location. The look-up table contains thermal model results such as ice-table vapor densities and average surface temperatures as functions of latitude, thermal inertia, albedo and ice depth. The scheme to generate these maps of stable ground ice is described in this section. 
To generate maps of stable ground-ice distribution we require values for latitude albedo, thermal inertia and elevation over the martian surface, and the annual average water column in some cases. The albedo and thermal inertia values used are results from the TES instrument, from Christensen et al. (2001) and Putzig et al. (2005) respectively. Maps of these values are shown in Figure 23 and Figure 24. Surface elevation is based on MOLA results (Smith et al. 2003). Average water column values for the present martian epoch come from Smith (2002). The thermal conductivity and density of the top, ice-free layer are both derived from thermal inertia values. We use a relation between thermal inertia and density defined by Mellon and Jakosky (1993); with this density and an assumed specific heat of the ground, thermal inertia can then be used to calculate thermal conductivity; $k=(\text { t.i. })^{2} / \rho c$.

We generate maps with a $1^{\circ}$ latitude by $1^{\circ}$ longitude resolution. Most datasets used have significantly higher spatial resolution than our maps, only the average water column values have lower resolution. Linear interpolation is used to find the $1^{\circ} \mathrm{x} 1^{\circ}$ water column values. Thermal inertia values from Putzig et al. (2005) extend from $80^{\circ} \mathrm{S}$ to $80^{\circ} \mathrm{N}$, which determines the extent of the stable ice maps we show here. 


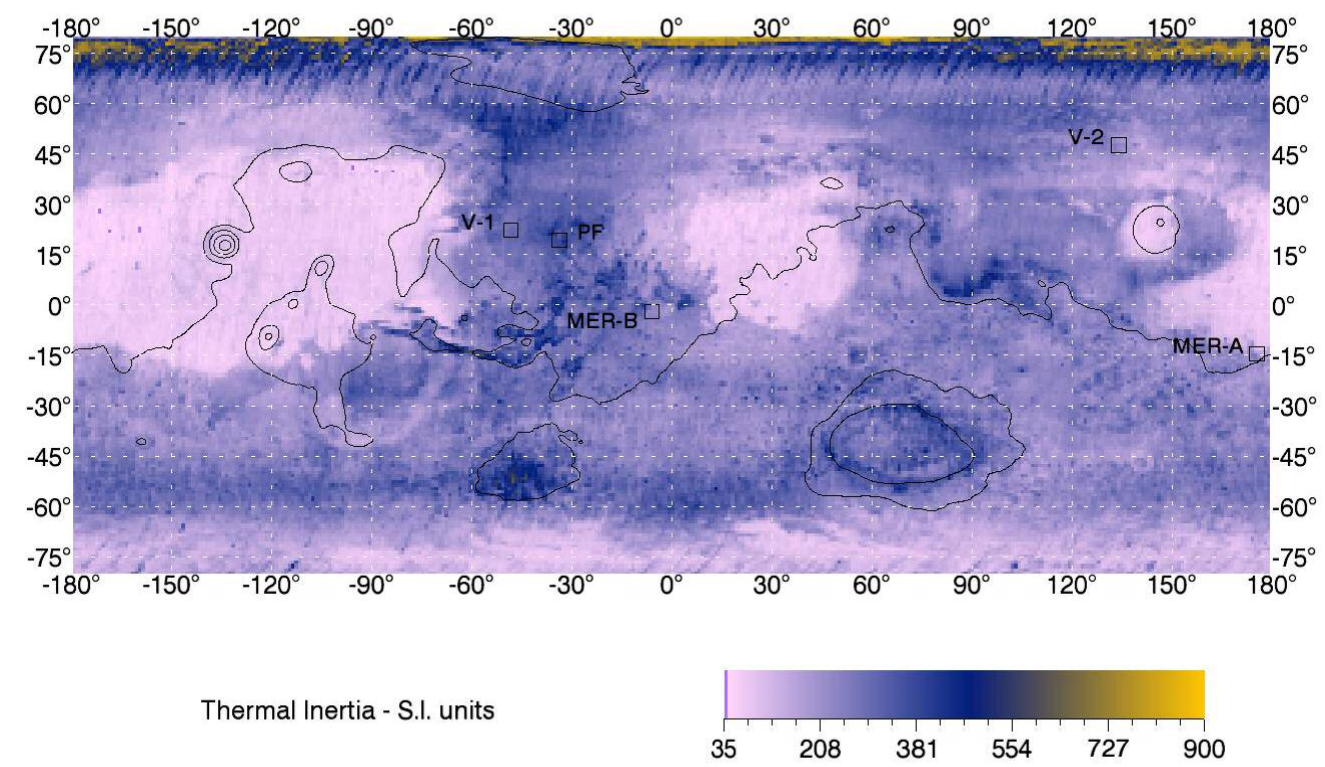

Figure 23 Martian thermal inertia map. The data used was published in Putzig et al. (2005).

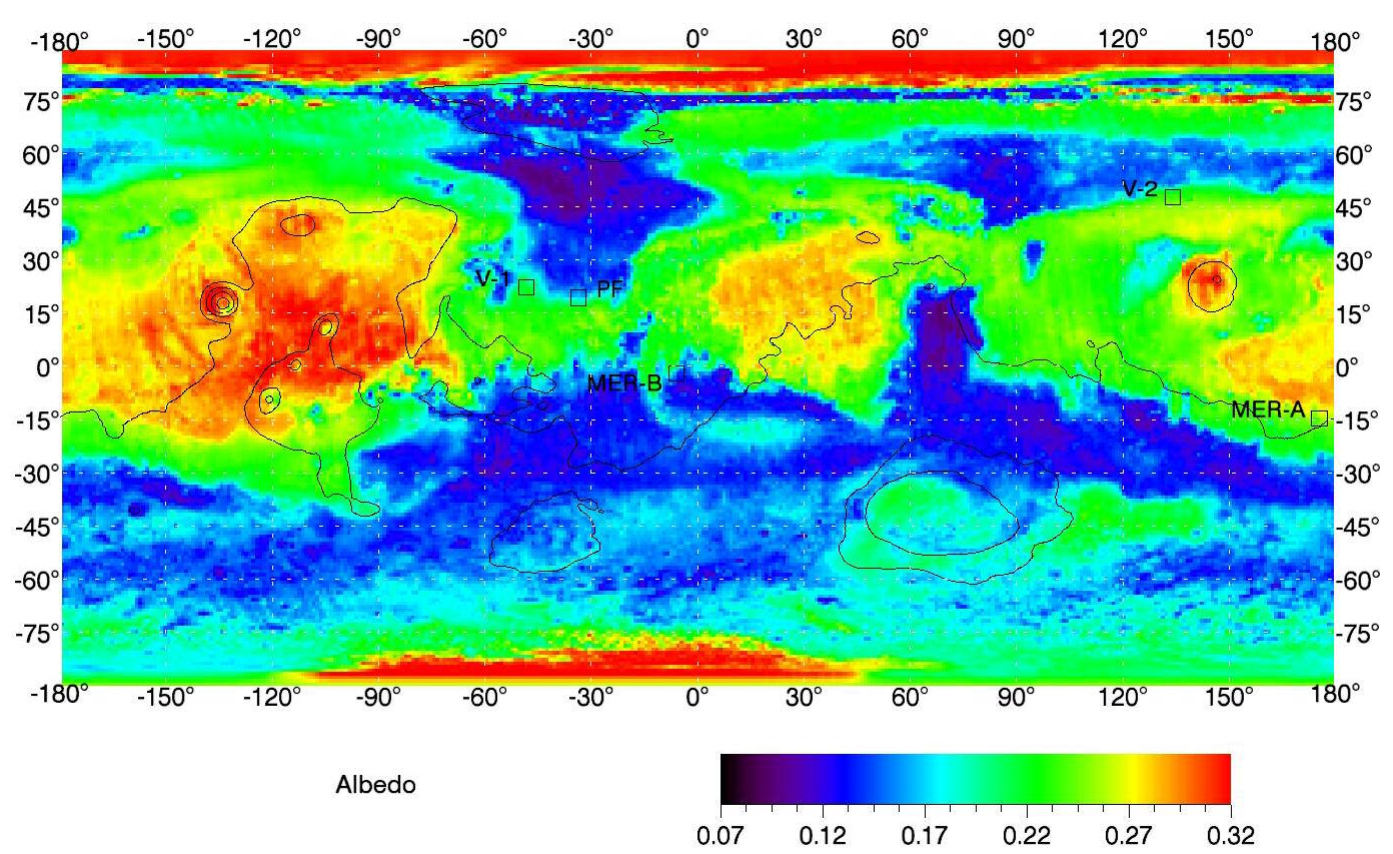

Figure 24 Martian albedo map. TES data was used to generate the map above (Christensen et al. 2001). 
When generating a look-up table the latitude spacing we use is $5^{\circ}$. The set of results from the thermal model are interpolated across latitudes using a cubic-polynomial fit to the four nearest latitude values. When interpolating to latitudes near the pole, latitudes are wrapped over the pole so that the interpolated values is always in the center of the four known latitude node values. Three albedo values are evaluated $(0.08,0.21$, 0.33 ) and are interpolated with a quadratic polynomial; it was found that results vary smoothly as a function of albedo. Models are run for thermal inertia values of 30, 60, 100, 200, 350, 600, and 1000 S.I. units, and results are interpolated with a cubic spline with respect to the $\log$ of the thermal inertia value. Effective water vapor density ratios, described in Section 2.2.3, are calculated for average water vapor column abundances from 0.032 to $32000 \mathrm{pr}-\mu \mathrm{m}$ in factors of 10 . The effective ratio used at a particular location is found by cubic spline interpolation with respect to the log of the average water vapor column abundances. Ice-table vapor densities are interpolated as logarithms of the density values, which were found to make a small improvement in the interpolation. Finally, interpolations are made with respect to the logarithm of ice depth, as described in Section 2.2.4.

Ice-table vapor densities are then compared to the effective water vapor density in the near-surface atmosphere to find where ice is stable. We typically use a uniform global average water vapor column abundance and correct this for elevation based on the MOLA topography. Alternatively, we can use the average water column observed by 
TES at different latitudes and longitudes (Smith 2002), but this can only be done for the present orbit.

The spacing of the node values of this grid and the interpolation schemes were designed so that contribution to the uncertainty in the annual average surface temperature is better than $0.1 \mathrm{~K}$ for each step of the process. Trial-and-error was employed to get to this stage.

To demonstrate how close we got to our goal, a series of thermal models were run to evaluate the effect of interpolation from the look-up table. Thermal models were run at $100+$ random coordinates in thermal inertia/albedo/latitude space. Results from these direct thermal models were compared to results found by interpolation to the same points. Figure 25 shows a comparison in the ice depth and average surface temperature between the direct thermal models and interpolation. This figure suggests that the interpolation process introduces an error usually no greater than a few percent in ice depth or $0.1 \mathrm{~K}$ for average temperature. Note that ice was not stable in every model tested for the atmospheric water content applied. 

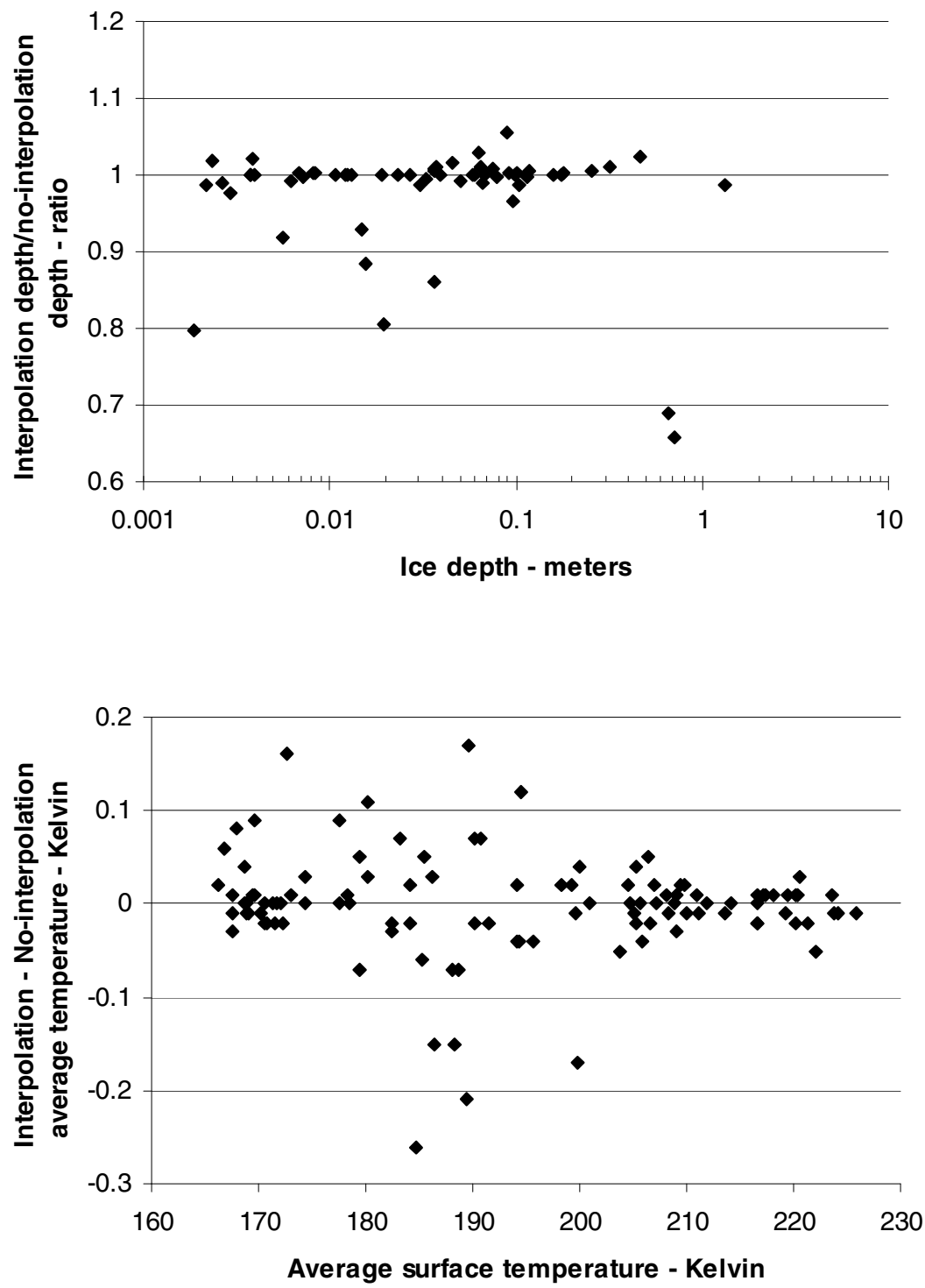

Figure 25 Difference in model results due to interpolation from the look-up table. Top panel shows the difference in the depth to ice for 100+ random points in thermal inertia-albedo-latitude space found by a direct thermal model and depths at the same points found by interpolation from the lookup table of results. The lower panel shows the differences in the average surface temperatures between the same sets of models shown in the top panel. 


\subsubsection{Climate at Other Epochs}

\subsubsection{1 $\mathrm{CO}_{2}$ as a Function of Obliquity}

How does $\mathrm{CO}_{2}$ vary with obliquity? The answer is at least related to the nature of the present south polar cap. Is the perennial $\mathrm{CO}_{2}$ stable at present or just residual from a previous climate? If perennial $\mathrm{CO}_{2}$ is stable, then at any lower obliquity we expect the $\mathrm{CO}_{2}$ to be reduced and controlled by the energy balance at the poles (or whichever pole has perennial $\mathrm{CO}_{2}$ ). If the $\mathrm{CO}_{2}$ over the southern cap is 'transient,' that is to say that the cap is about to disappear, there should still be an obliquity limit below which perennial $\mathrm{CO}_{2}$ will be become stable at one pole or another due to the reduction in insolation received and lower temperatures at the poles as obliquity decreases. At obliquities greater than this limit, the atmospheric pressure should be about what is observed today. We assume here that the present obliquity of Mars represents this limit, so that energy balance at the poles controls $\mathrm{CO}_{2}$ in the atmosphere for obliquities less than present and the $\mathrm{CO}_{2}$ in the atmosphere is about constant for higher obliquities.

The growth of holes in 'Swiss Cheese' terrains indeed suggest the $\mathrm{CO}_{2}$ may be close to disappearing (Malin et al. 2001). The thinness of the $\mathrm{CO}_{2}$ mantle $(\sim 10 \mathrm{~m})$ indicates that the mass of present perennial $\mathrm{CO}_{2}$ is small with respect to the mass of the atmosphere; the mass of the atmosphere would not change significantly if all this perennial $\mathrm{CO}_{2}$ sublimed. 
The significance of the atmospheric thickness on Mars is that it will modify the surface temperatures. Seasonal $\mathrm{CO}_{2}$ frost forms poleward of the mid latitudes in the present epoch; when seasonal frost builds up on the surface the surface temperature is buffered at the frost point and this temperature is a function of surface pressure. Also, the thickness of the atmosphere will modify the attenuation of insolation and the amount thermal radiation received at the surface. Dust in the atmosphere is active in the visible and infrared. We assume the dust content of the atmosphere at any epoch is proportional to the surface pressure; at present the average dust opacity is taken as $\tau=0.3$ and pressure is $610 \mathrm{~Pa}$.

For this work, we use a simple energy balance to estimate the atmospheric pressure in past epochs with low obliquity. We estimate the atmospheric pressure of Mars by balancing the polar insolation with the thermal radiation at the $\mathrm{CO}_{2}$ frost temperature, based on the approach used by Leighton and Murray (1966). This frost temperature is a direct function of atmospheric pressure. The annual average insolation received at the poles is a function of eccentricity $(e)$ and obliquity $(\Theta)$ of the planet in the equation, $\langle I\rangle=\frac{I_{s c} \sin \Theta}{\pi \sqrt{1-e^{2}}}$,

as shown by (Murray et al. 1973) where $I_{s c}$ is the solar constant at the planet's semi-major axis. Where a polar $\mathrm{CO}_{2}$ cap is stable or perennial, this average polar insolation is balanced by the thermal radiation that can be related to the atmospheric pressure of Mars. When radiation is balanced there is no net annual change in the surface $\mathrm{CO}_{2}$ frost 
reservoir. Equating the thermal radiation to the average polar insolation gives the relation,

$(1-A)\langle I\rangle=\varepsilon \sigma T_{\mathrm{CO}_{2}}^{4}$, which gives the general relation,

$T_{\mathrm{CO}_{2}} \propto \sin ^{1 / 4} \Theta$

after ignoring eccentricity terms which are not significant, even for Mars' orbit. $A$ and $\varepsilon$ are the albedo and emissivity, respectively, of the surface of the perennial $\mathrm{CO}_{2}$, and $\sigma$ is the Stefan-Boltzmann constant.

For our simple approach, we can use the present orbit to estimate the $\mathrm{CO}_{2}$ frost temperature at other obliquities using $\Theta_{\text {present }}=25.19^{\circ}$ and $T_{C O 2 \text {, present }}=145.0 \mathrm{~K}$, using a frost temperature for the high elevation southern cap. Now temperature as a function of obliquity can be estimated by,

$T(\Theta) \approx 145.0 \frac{\sin ^{1 / 4} \Theta}{0.80771}$

Atmospheric pressure can then be estimated as the vapor pressure of $\mathrm{CO}_{2}$ frost at this temperature with $\mathrm{P}_{\mathrm{CO} 2}=1.382 \mathrm{e} 12 \exp (-3183 / \mathrm{T})$, using the relation used within the Ames GCM (e.g. Haberle and Jakosky 1991). This simple treatment neglects many processes: 1. atmospheric circulation and advection of heat, 2. what happens if residual $\mathrm{CO}_{2}$ is at the other pole at a different elevation, 3. what if the $A$ or $\varepsilon$ of the $\mathrm{CO}_{2}$ frost vary, 4. effects adsorption of $\mathrm{CO}_{2}$ within the regolith of Mars, and, 5. what happens with minor constituents of the atmosphere (at obliquities of $\sim 15^{\circ}$ the martian atmosphere may become dominated by $\mathrm{N}_{2}$ and $\mathrm{Ar}$ ). However, these factors are poorly constrained and we just want a rough pressure value to use and the atmospheric thicknesses we calculate with 
this scheme are consistent with some other works. Surface temperatures are not very sensitive to surface pressure, in part, because of the thin atmosphere. Kieffer and Zent (1992) also produced a simple model of atmospheric pressure as a function of orbit and obliquity and they explored the effect of some of the processes listed above; they show results that are qualitatively the same as ours. Zent and Quinn (1995) allowed $\mathrm{CO}_{2}$ to be adsorbed within the regolith and found atmospheric pressures at high obliquity were higher by a factor of a few, depending on model parameters; but at least the differences were not an order of magnitude. However, other papers have indicated average surface pressure may decrease at high obliquities due to a combination of adsorption in the regolith and large seasonal pressure cycles (Armstrong et al. 2004; Haberle et al. 2003). At obliquities higher than the present Mars, if a polar $\mathrm{CO}_{2}$ cap is still permanent this would increase the atmospheric pressure due to increased insolation received at the poles. However, it is generally thought that this would not be the case as the perennial $\mathrm{CO}_{2}$ would be rapidly depleted (e.g., Jakosky et al. 1993). At high obliquities, seasonal variations would be enhanced, so while permanent $\mathrm{CO}_{2}$ caps would retreat and disappear, the seasonal $\mathrm{CO}_{2}$ caps would become more extensive. As a result the seasonal pressure variation would be greater than what was observed by the Viking Landers. For this project, we assume the atmospheric pressures at higher obliquities are equal to the present Mars. A figure showing the value of $\mathrm{CO}_{2}$ pressure as a function of obliquity used in this project is shown in the Section 2.3.2.2 that discusses results from varying obliquities. 


\subsubsection{Water Vapor at Other Epochs}

In order to determine the distribution of ground ice that is in diffusive contact with the atmosphere, it is necessary to know the seasonal temperatures of the surface and the water content of the atmosphere. Surface temperatures at other epochs are found using the same thermal model that is used for the present epoch, with the exception that the inputs for orbit, obliquity and atmospheric pressure are varied as described in the previous sections. The atmospheric water content at other epochs is just as significant in determining the extent of stable ground ice at these times. Here we propose a new idea to quickly estimate the average atmospheric water content at other epochs.

Previously, atmospheric water content has been estimated by the seasonal sublimation for surface ice at the polar cap (Mellon and Jakosky 1995; Jakosky et al. 1993). This process assumes that the residual cap sublimes into a dry, understaturated atmosphere. GCMs that include the water cycle have also been run at high obliquities which provide estimates of atmospheric water contents at particular epochs.

We propose instead to use the saturated water vapor column abundance over ground with the properties of surface ice as a way of estimating water content. While calculating the depletion of water vapor near the surface as described in Section 2.2.3, we regularly calculated the maximum water content of the atmosphere (or water carrying capacity), of both the whole atmosphere and the near-surface. It was noticed that the water carrying capacity over the north pole for the present Mars closely matched the present observed water content and that it can be calculated relatively easily - certainly 
easier than coding up a full GCM. This observation also suggests that the atmosphere over the residual cap is actually saturated, or close to it, at all times and responds rapidly to the temperature of the surface.

When estimating the global water vapor content of Mars at different epochs, the atmospheric water carrying capacities are calculated over surface ice at each pole and at latitudes $10^{\circ}$ apart. These water capacities are corrected for the elevation of different latitudes; this has the effect of increasing the effective carrying capacity, or water content, from surface ice at high elevations. The location with the lowest corrected water carrying capacity is then used for the global water content. The idea is that locations where the atmosphere has the lowest water carrying capacity will act as a sink and eventually dominate the global content. Locations with surface ice where the atmosphere has a high carrying capacity become sources of water vapor until this surface ice is depleted and/or becomes an ineffective source to the atmosphere. In the present epoch, the global atmospheric water content of Mars is the same as the location with the lowest water carrying capacity, which is over the north pole. At obliquities lower than $30^{\circ}$, the carrying capacities over the poles are less than the capacity of the lower latitudes. Because the south pole experiences summer near perihelion in the present epoch, the carrying capacity in the south is much greater than the north; so it is the north pole that has the minimum capacity and it is this location that determines the global atmospheric water content. If substantial areas of water ice were exposed at the south pole in the present epoch, it would supply more water to the atmosphere than ice in the north can, but it would deplete the ice available at the surface in the south as well. 
Figure 26 shows the water vapor carrying capacity of the atmosphere over the north pole for the present epoch as a function of the albedo and thermal inertia of the residual water ice cap. The north polar cap is at a low elevation with respect to the rest of Mars, so for Mars to have an average water column of $14.1 \mathrm{pr}-\mu \mathrm{m}$ and the north pole the column must be $17.4 \mathrm{pr}-\mu \mathrm{m}$. This target column abundance is achieved with a combination of an albedo of 0.34 and a thermal inertia of 1350 S.I. units, which compare well with results from Paige et al. (1994) and Kieffer and Titus (2001). Note that this is not a unique combination to reproduce the present water content.

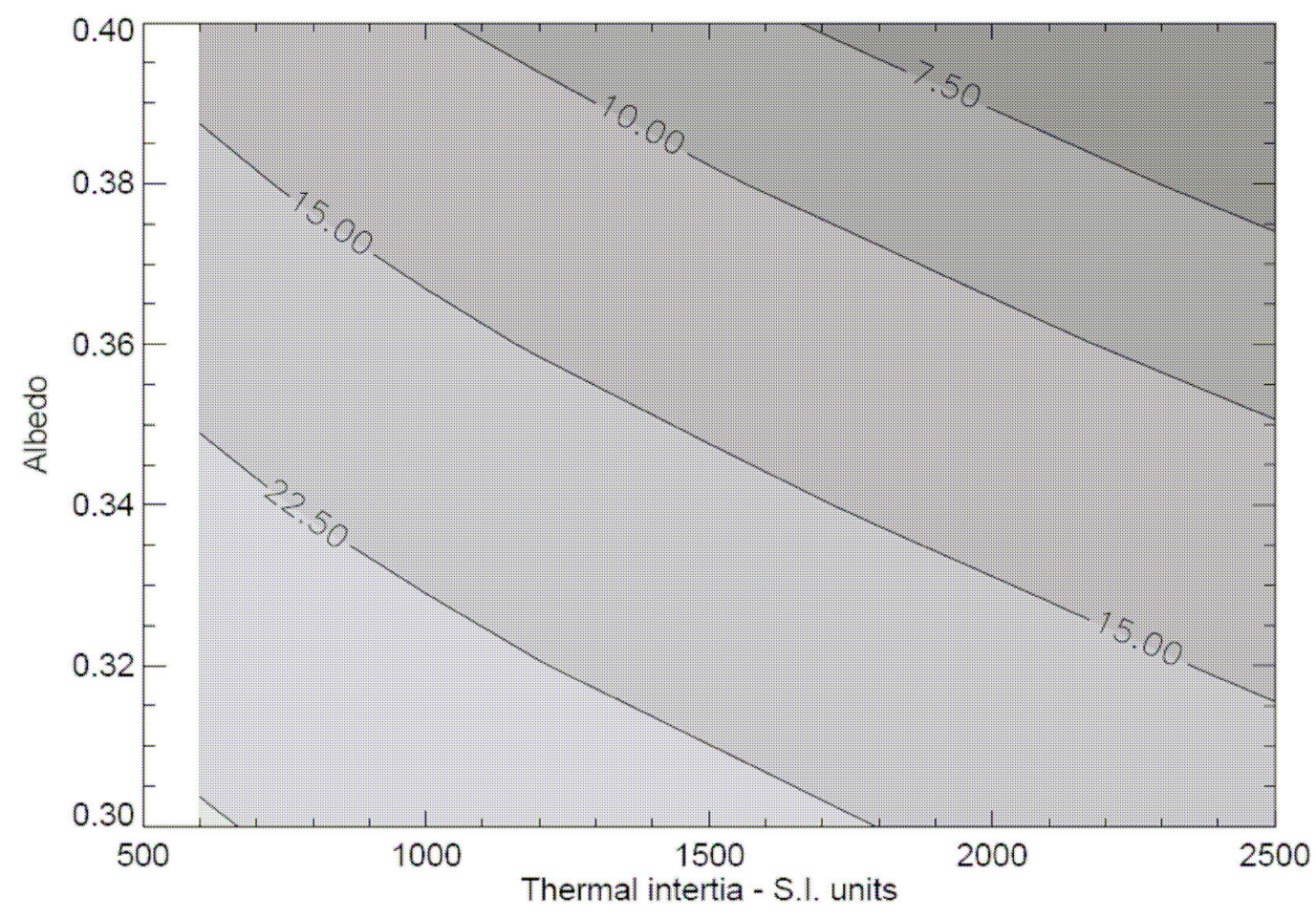

Figure 26 Water carrying capacity of the atmosphere over the north pole in the current orbit of Mars. We take the average water content of Mars to be $14.1 \mathrm{pr}-\mu \mathrm{m}$ for the present epoch of Mars. The elevation of the top of the north residual cap is $\sim-2200 \mathrm{~m}$; adjusting the water content to this elevation gives $17.4 \mathrm{pr}-\mu \mathrm{m}$. Based on the results above, we assume that water ice on the surface has an albedo of 0.34 and 1350 to match the observed atmospheric water content. 
Figures showing the average atmospheric water content as functions of obliquity and the position of perihelion, and corresponding discussions are included in the respective sub-subsections of the results, Section 2.3.2.

\subsection{Results}

The models described above in Section 2.2 are used to generate several maps of ground-ice distribution. The first maps below show the estimated distribution of stable ground ice on Mars for the present epoch. Following these maps is a discussion of the effect ground ice has on surface temperature. We then test how various components of the models affect the ice distribution. We then explore how the stable ice distribution would vary while Mars is still in its present orbit but with varying amounts of water in the atmosphere. Section 2.3.2 explores the effect of modifying the orbit, first changing the position of perihelion and then changing the obliquity, testing where ground ice is expected to have been present over the geological history of Mars. Table 4 summarizes all the ground-ice distributions generated below. The table shows average latitudinal extents of ground ice for each hemisphere and average ice depths for selected latitude bands. This allows rapid comparisons of the extent of ground ice in response to the orbit and other processes. Also included in Table 4 are the base atmospheric water contents used to generate each map. 


\begin{tabular}{|c|c|c|c|c|c|c|c|}
\hline \multirow[t]{2}{*}{ Map } & \multirow{2}{*}{$\begin{array}{l}\text { Atmo. } \\
\mathrm{H}_{2} \mathrm{O} \\
\text { pr- } \mu \mathrm{m}\end{array}$} & \multicolumn{2}{|c|}{ Lat limit $-{ }^{\circ} \mathbf{N}$} & \multicolumn{4}{|c|}{ Depth to ice - cm } \\
\hline & & Sth. & Nth. & $70 \mathrm{~S}$ & $60 \mathrm{~S}$ & $60 \mathrm{~N}$ & $70 \mathrm{~N}$ \\
\hline present_best & 14.1 & -46.3 & 47.0 & 1.87 & 10.40 & 8.44 & 3.87 \\
\hline th_corr & 14.1 & -45.4 & 46.4 & 1.80 & 9.61 & 7.48 & 3.63 \\
\hline present_typical & 14.1 & -45.3 & 46.6 & 1.76 & 9.48 & 8.04 & 4.34 \\
\hline present_no_vap_dpl & 14.1 & -44.1 & 40.4 & 1.57 & 8.24 & 5.72 & 3.10 \\
\hline present_no_atmo_elev & 14.1 & -45.2 & 47.9 & 1.69 & 9.06 & 10.42 & 5.88 \\
\hline present_2percent & 14.1 & -48.0 & 48.5 & 1.30 & 6.82 & 6.44 & 3.79 \\
\hline k15 & 14.1 & -45.3 & 46.6 & 2.12 & 11.19 & 9.73 & 5.46 \\
\hline $\mathrm{k} 35$ & 14.1 & -45.3 & 46.6 & 1.58 & 8.57 & 6.93 & 3.64 \\
\hline 1pr_microns & 1 & -56.8 & 58.7 & 4.37 & 33.66 & & 17.77 \\
\hline 2pr_microns & 2 & -53.5 & 55.8 & 3.59 & 21.77 & 26.38 & 13.39 \\
\hline 5pr_microns & 5 & -49.5 & 52.1 & 2.56 & 13.93 & 15.21 & 8.73 \\
\hline 10pr_microns & 10 & -46.6 & 48.8 & 2.00 & 10.66 & 10.44 & 5.76 \\
\hline 20pr_microns & 20 & -43.9 & 44.6 & 1.57 & 8.26 & 6.38 & 3.34 \\
\hline 50pr_microns & 50 & -40.0 & 35.7 & 1.08 & 5.39 & 2.85 & 1.52 \\
\hline 100pr_microns & 100 & -32.8 & 32.5 & 0.72 & 3.62 & 2.23 & 1.74 \\
\hline $\mathrm{e} 00$ & 24.9 & -46.1 & 38.5 & 1.27 & 7.39 & 5.97 & 3.76 \\
\hline peri000 & 22.8 & -46.3 & 39.0 & 1.44 & 8.23 & 6.04 & 3.82 \\
\hline peri270 & 12.0 & -45.6 & 47.7 & 1.89 & 10.05 & 8.60 & 4.55 \\
\hline peri180 & 35.6 & -44.3 & 34.2 & 1.04 & 5.84 & 5.30 & 3.54 \\
\hline peri090 & 22.5 & -49.7 & 35.4 & 1.07 & 7.36 & 6.72 & 4.95 \\
\hline ob10 & 0.0087 & -73.2 & 73.7 & & & & \\
\hline ob15 & 0.33 & -64.8 & 64.0 & 4.41 & & & 16.12 \\
\hline ob20 & 4.0 & -57.1 & 53.9 & 1.89 & 21.41 & 16.87 & 6.49 \\
\hline ob25 & 20.5 & -47.0 & 40.7 & 1.35 & 8.08 & 6.57 & 4.06 \\
\hline ob30 & 100 & -35.3 & 23.7 & 1.15 & 4.41 & 3.38 & 3.14 \\
\hline ob35 & 128 & -30.2 & 19.3 & 1.73 & 5.59 & 4.45 & 5.29 \\
\hline ob40 & 110 & -27.2 & 16.9 & 2.64 & 8.25 & 6.92 & 8.93 \\
\hline ob45 & 94 & -22.6 & 13.8 & 3.72 & 10.99 & 9.55 & 12.71 \\
\hline
\end{tabular}

Table 4 Summary of ground-ice distribution for different models. Base atmospheric water refers to the water content of the atmosphere at zero MOLA elevation. The limits shown are the average latitudinal extent of the ground ice in each hemisphere, negative values represent southern values. The average depths to ground ice and average annual surface temperatures are shown for bands of $1^{\circ}$ width in latitude. For simplicity, the depth value is left blank when ground ice is not present across the whole band. 


\subsubsection{Present Mars}

Figure 27 below shows our present best estimate for the distribution of martian ground ice that is stable in the present epoch. This is summarized as model "present_best" in Table 4. To generate this figure, the temperatures from the thermal model have been adjusted for the surface pressure and dust opacity of the atmosphere, as functions of elevation. Also, the average water content of the atmosphere used in Figure 27 is based on the water column observed by TES; we interpolate the elevation-corrected data from Smith (2002) to the resolution of our map and adjust the water content for the MOLA elevation. In addition, we apply our vapor depletion of the near-surface atmosphere to correct for the reduction in the average vapor density due to nighttime frost in the atmosphere just above the surface with which any ground ice, if present, will be in diffusive contact. 


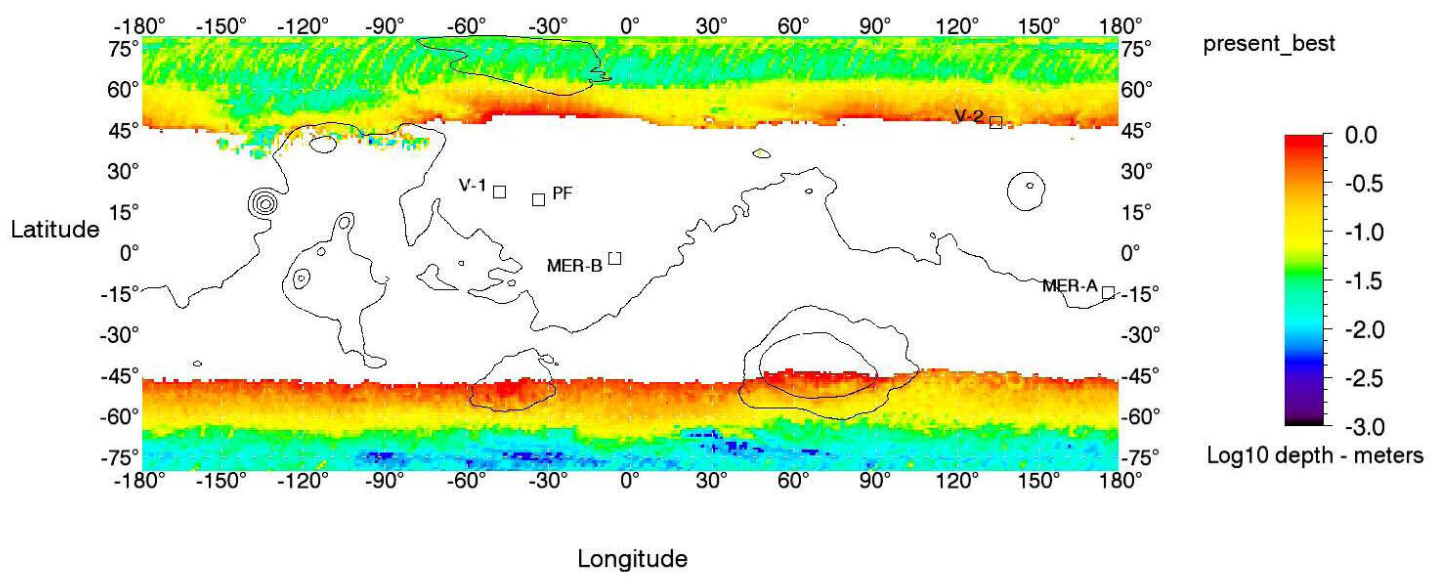

Figure 27 Best estimate of depth to stable ground ice on Mars in the present epoch. An elevation correction to the surface temperatures and observed atmospheric water contents are applied. Ice is at a depth such that there is zero net flux in vapor diffusion to the atmosphere over the season. Water vapor is allowed to be depleted in the near-surface atmosphere by the diurnal temperature cycle. Coarse, $5 \mathbf{~ k m}$ elevation contours and successful lander sites are shown on this and following figures for orientation.

Note that the procedure used to generate Figure 27 is not the same as that used for maps at other epochs. Firstly, there are no observations of the atmospheric water content over the martian globe at other epochs. Secondly, we find the adjustment to the temperatures due to elevation and atmospheric thickness is very minor and so we do not routinely calculate them. Figure 28 (model "present_typical" in Table 4) shows the expected distribution of ground ice for Mars in the present epoch using the same procedure typically used for other epochs. A uniform, base atmospheric water content of 14.1 pr- $\mu \mathrm{m}$ (adjusted for elevation) is assumed and the thermal model results are not 
corrected for atmospheric thickness or elevation. The depletion of water in the nearsurface atmosphere due to the diurnal temperature cycle is still included. The slight differences between the ice distribution in Figure 27 and Figure 28 are discussed later in this section. The distribution is essentially the same as maps shown in Mellon et al. (2004) which agree well with the distribution of ground ice observed with hydrogen gamma ray and neutron fluxes (Feldman et al. 2004b; Boynton et al. 2006). This agreement indicates that our models that we will use at other epochs reliably reproduce the ground-ice distribution for the present epoch.



Figure 28 The distribution of martian ground ice for the present epoch using the same typical procedure that is applied in other epochs. The base atmospheric water content is $14.1 \mathrm{pr}-\mu \mathrm{m}$.

Figure 29 shows the average temperature of the surface of Mars in the present epoch. Temperatures come from the same thermal model results and atmospheric water 
content used in Figure 27 which shows our best estimate of the present distribution of ground ice. As a first approximation, the distribution of ground ice closely follows the locations of Mars that have an average surface temperature less than the frost point for atmospheric water ( 196 K). Figure 29 also shows the change in the average annual temperature due to presence of ground ice. There are significant increases in the average surface temperature at high latitudes where ground ice with high thermal inertia is very close to the surface, well within the seasonal thermal wave. At these high latitudes the ground ice heats the surface several degrees with respect to the average temperature if ice were not present.

While we are discussing the effect of ground ice on surface temperatures of Mars, it is as good a place as any to mention an effect ground ice has on average surface temperatures and Crocus dates of high martian latitudes. By Crocus dates, we refer here to the time of year that a particular location loses the last of its seasonal $\mathrm{CO}_{2}$ frost, indicated by surface temperatures rising significantly above the frost point. This time may or may not be the same as the time when the location looses its high-albedo frost cover (as observed by cameras in orbit). 


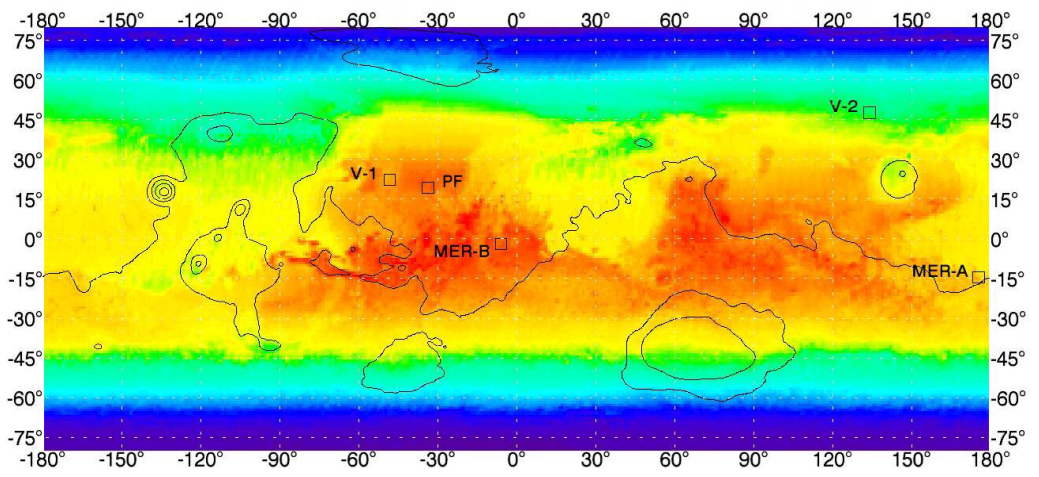

Average surface temperature - Kelvin
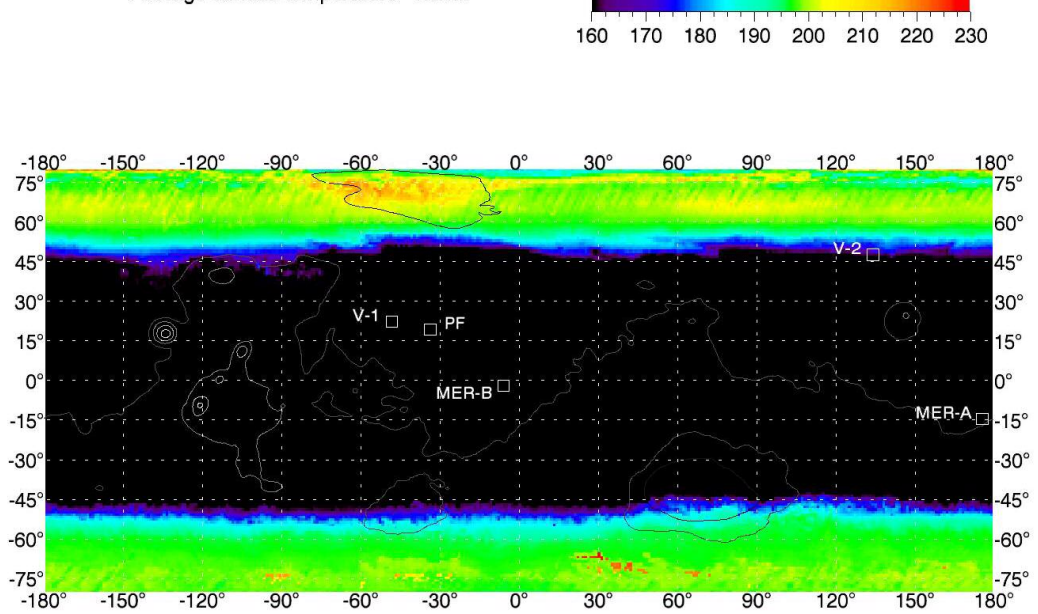

Difference in average surface temperature - Kelvin

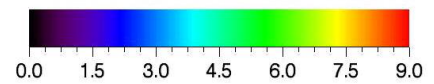

Figure 29 The average surface temperature of Mars in the present epoch, and the change in this temperature due to the presence of near-surface ice, relative to the temperature if no ice were present and the thermal properties were uniform with depth. 
Where ground ice is not stable on Mars, average surface temperatures should be low for low-thermal inertia material and high for high-thermal inertia material. A simple way to explain why this works is that low thermal inertia material reaches higher temperatures during the day so more energy is lost from the ground as thermal radiation. High-thermal inertia material does not get as hot during the day and more of the heat is conducted into the ground raising the temperature. High-thermal inertia material is warmer overnight with respect to low-thermal inertia material, though the temperature to the 4th power dependence of thermal radiation makes the daytime temperature difference more significant. The reason we elaborate this point here is that at high latitudes where ground ice is present, the presence of ground ice and its stable depth work to almost negate this thermal inertia effect on average temperature.

The middle panel of Figure 30 shows the average surface temperatures for models at $70^{\circ} \mathrm{N}, 0.20$ albedo and varying thermal inertia. The two curves in this panel show the average annual surface temperatures of the ground with and without ground ice. The depth to ground ice is set so that it is stable to the same base content of water in the atmosphere, and this depth is shown in the top panel. Note that the variation of almost $15 \mathrm{~K}$ across the thermal inertia values shown for ice-free ground is reduced to just a few degrees when ground ice is included. Ground ice is closest to the surface when the top layer has the lowest thermal inertia which has the effect of raising the effective thermal inertia of the model over the season. 
Like the variation in average surface temperatures, the lower panel of Figure 30 shows that the presence of ground ice also negates the variation in the Crocus date due to thermal inertia of the top layer. The Crocus date is one of few observables for the thermal properties of Mars, though the combination of water and $\mathrm{CO}_{2}$ frost complicates what the Crocus date means. Also, the maximum amount of $\mathrm{CO}_{2}$ frost that builds up on the surface becomes much less dependent on the thermal inertia of the top layer when ground ice is included, which has significant implications for GCMs. We find that the average surface temperature, Crocus date and maximum seasonal $\mathrm{CO}_{2}$ for any particular high-latitude location are all more sensitive to the effective water content of the atmosphere, which determines the depth to stable ice, than the thermal inertia of the top layer.

The atmospheric parameters used to produce the results shown in Figure 30 are typical values, i.e. "ob25,e09,p610” parameters from Table 2. We did not account for the thick atmosphere at the latitude of the thermal model tested $\left(70^{\circ} \mathrm{N}\right)$ so the model Crocus dates are earlier than those observed at this latitude on Mars. The change in albedo is seen by the MGS-Mars Orbiter Camera images (James and Cantor 2001) and the temperature increase is seen by TES (Kieffer and Titus 2001)). Also, model amounts of seasonal $\mathrm{CO}_{2}$ frost are less than observed with the gamma ray attenuation (Kelly et al. 2006). However, we are more interested here in demonstrating the process rather than matching data for the moment. Ultimately a thermal model that is consistent with all the available data should be produced. 

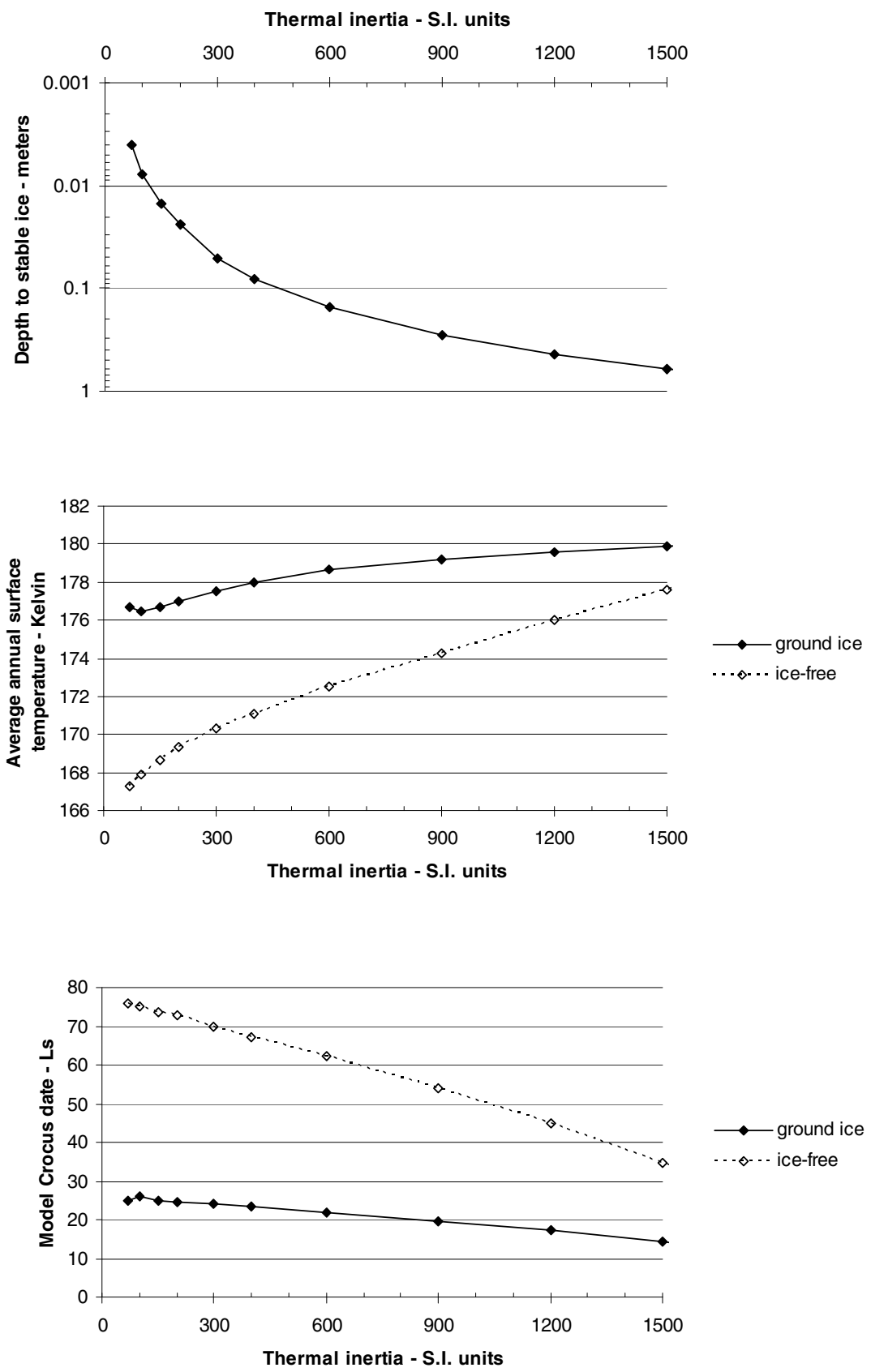

Figure 30 The effect of ground ice on model results at high latitudes as a function of thermal inertia. Results are shown for models at $70^{\circ} \mathrm{N}$, an albedo of 0.20 and varying thermal inertia, both with and without ground ice. The depth of ground ice in each case was such that the ice would be in steadystate with a mean atmospheric water content of $14 \mathrm{pr}-\mu \mathrm{m}$. The $\mathrm{x}$-axis is the thermal inertia of top, ice-free layer; the ground has high-thermal inertia properties at depths where ice is present. Note that the presence of the ground ice makes the model results relatively invariant to the thermal inertia of the top layer. 


\subsubsection{Discussion of model components}

A series of different maps are generated to test the sensitivity of the various aspects of the models used to determine the distribution of the stable ground ice. The maps themselves are not shown, indeed it often hard to notice the difference between them and the maps that have already been shown. Summaries of the extent and depth of ground ice are however included in Table 4.

A couple of maps have been presented already. The "present_typical" map shows the stable ice distribution for the present epoch using the typical procedure that will used to generate maps at other epochs. The "present_best" map is our best estimate of the present extent of stable ice and includes two extra procedures not included in the "present_typical" map. The extra procedures are: a correction to the thermal model for elevation (which slows the computation) and the use of the observed atmospheric column (which is only available for the present epoch). To isolate the contribution of each of these components to the ice distribution, another map "th_corr" was generated that includes the same thermal correction for elevation as the "present_best" map, but uses the same uniform base atmospheric water content of "present_typical." The same thermal model look-up table is used for the 2 maps, "present_best" and "th_corr," only different atmospheric water abundances are used.

The contribution of the thermal radiation from the atmosphere and attenuation of insolation are both functions of atmospheric pressure which is related to elevation. We 
run the detailed atmospheric model with pressure and opacity corresponding to elevations $-4000 \mathrm{~m}$ and $+4000 \mathrm{~m}$. We then use these detailed-model results to retune our atmospheric model (parameters “ob25,e09,p910” and “ob25,e09,p409” in Table 2) and generate new look-up tables. A new, combined, look-up table is formed with the results of different elevations and allows us to interpolate with respect to elevation. Interpolation is done in log-pressure space, or linearly in elevation space.

The effect of the elevation correction to the thermal model is seen by comparing summaries of maps "th_corr" and "present_typical" in Table 4. Comparing the summaries of the maps "th_corr" and "present_best" shows the effect of using the observed water column observed by Smith (2002). The effect of using the observed water column is more significant than the effect of the elevation correction to the thermal model. The Smith (2002) water content reduces the latitudinal extent of ice by of the order of $1^{\circ}$ in each hemisphere while the elevation correction to the thermal model only changed the latitudinal extent by $0.1^{\circ}$.

The effect of the water-vapor depletion of the near-surface atmosphere can be seen by comparing the maps "present_no_vap_dpl" and "present_typical." These maps both use the same thermal model results, it is only the water vapor density of the nearsurface atmosphere that is modified. By comparing the maps we see that including the vapor depletion of the near-surface makes ground ice retreat from the equator. The amount of retreat is different for the 2 hemispheres. In the north the limit of the ground ice retreats several degrees of latitude while in the south it is only a couple of degrees. There are at least a couple of reasons to explain this asymmetry. The mid latitudes in the 
northern hemisphere have a lot of low thermal inertia regions which are more susceptible to vapor depletion due to a larger diurnal temperature range. Also, the higher summer temperatures in the southern hemisphere can reduce the depletion factors. Both of these reasons reduce the effect vapor depletion has in the south relative to the north. There is also a change in the ice depth; depletion of water vapor increases the depth at which ice is stable. There is also an asymmetry in the response of ice depth in the two hemispheres, the increase in ice depth in the north with vapor depletion is of the order of $50 \%$, while in the south it is only $\sim 15 \%$.

How important is the elevation correction to water content? Model "present_no_atmo_elev" was calculated where the water column is not adjusted for the elevation of the surface. This is the correction that reduces the atmospheric water content for high elevations such as Tharsis, and increases the water content at low locations like Hellas Basin. There is no significant change in ground ice extent in the south and an advance of $\sim 1.5^{\circ}$ latitude in the north when the atmospheric water content is adjusted for elevation. When the water content is corrected elevation ice becomes a little deeper in the south, less than $10 \%$, and $\sim 25 \%$ more shallow in the north where low elevations increase the local water content with respect to the base level.

Not many details are known about the ice-rich layer. For example, the porosity of the ground and ratio of ice and silicate are uncertain and probably vary regionally. However, the estimated values of thermal properties should be close to actual values, certainly good to within a factor of a few, since there is not a large range in property values for probable materials (e.g., ice, rock or a mixture of these). This may not seem 
very precise; however the significance of the ice-rich layer is that it has a thermal inertia that is orders of magnitude greater than the topmost ice-free layer. Small changes in the properties of the ice-rich layer are unlikely to modify the ice depth maps we generate. To illustrate this point we have run thermal models with different values of thermal conductivity, and hence different thermal inertia, for the ice-rich layer to produce new look-up tables. We consider conductivity values of $1.5 \mathrm{~W} \mathrm{~m}^{-1} \mathrm{~K}^{-1}$ and $3.5 \mathrm{~W} \mathrm{~m}^{-1} \mathrm{~K}^{-1}$ in addition to the typical value of $2.5 \mathrm{~W} \mathrm{~m}^{-1} \mathrm{~K}^{-1}$ that has been used for standard models. Results are summarized in Table 4 as models "k15" and "k35." These results show that the extent of the ground ice is unchanged and there are only minor changes to the ice depth. When the ice-rich layer is given a thermal conductivity of $3.5 \mathrm{~W} \mathrm{~m}^{-1} \mathrm{~K}^{-1}$ ice is stable closer to the surface, ice is more shallow by of the order of 10 to $20 \%$ with respect to the depths with $2.5 \mathrm{~W} \mathrm{~m}^{-1} \mathrm{~K}^{-1}$. When the ice-rich layer is given a thermal conductivity of $1.5 \mathrm{~W} \mathrm{~m}^{-1} \mathrm{~K}^{-1}$, ice is deeper by of the order of 20 to $25 \%$.

For the sake of comparison, we ran the thermal model using the " $2 \%$ assumption" to estimate the thermal radiation from the martian atmosphere. This approximation has been used a lot in the past few decades. Comparing "present_2percent" to "present_typical" shows that using the "2\% assumption" makes stable ground ice less extensive by of the order of 2 to $3^{\circ}$ and deeper by of the order of 10 to $20 \%$. 
2.3.1.2 Response of ground ice to changes in atmospheric water content.

The previous section discussed the effects that various aspects of the model have on the final ground-ice distribution that is calculated for Mars. For this previous discussion and the maps that were generated, Mars was in its present orbit and with the present amount of water in the martian atmosphere. Now we consider what happens when Mars is kept in its current orbit and the water content of the atmosphere is modified. Jakosky et al. (2005) suggest that Mars could have experienced periods of high water vapor content recently if the $\mathrm{CO}_{2}$ cap over the south pole had exposed the water ice underneath it during the summer as it does in the north every year. Note that this has not yet been observed to occur in the south while there have been active spacecraft keeping a close eye on the planet. Though, the southern summer season has been identified as the season that shows the greatest interannual variability (e.g., Smith 2004). Jakoksy et al. (2005) propose that an epoch with a high water content in the martian atmosphere could have formed near-surface low-latitude ice deposits that may explain observed neutron fluxes (Feldman et al. 2002; Feldman et al. 2004b).

Here we produce maps with different atmospheric water vapor abundances applied to the same thermal model results that were used for "present_typical" map. These maps show changes to the ground-ice distribution as a function of atmospheric water vapor. Results of this are shown in Figure 31 and summarized in Table 4 as models "*pr_ums." 



Log10 depth - meters

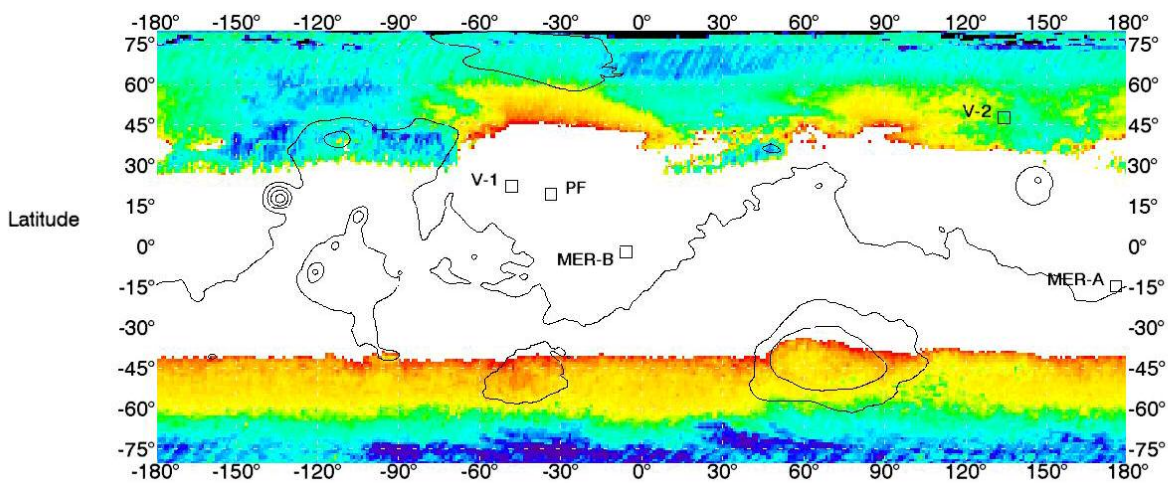

50pr_microns

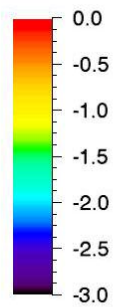

Log10 depth - meters
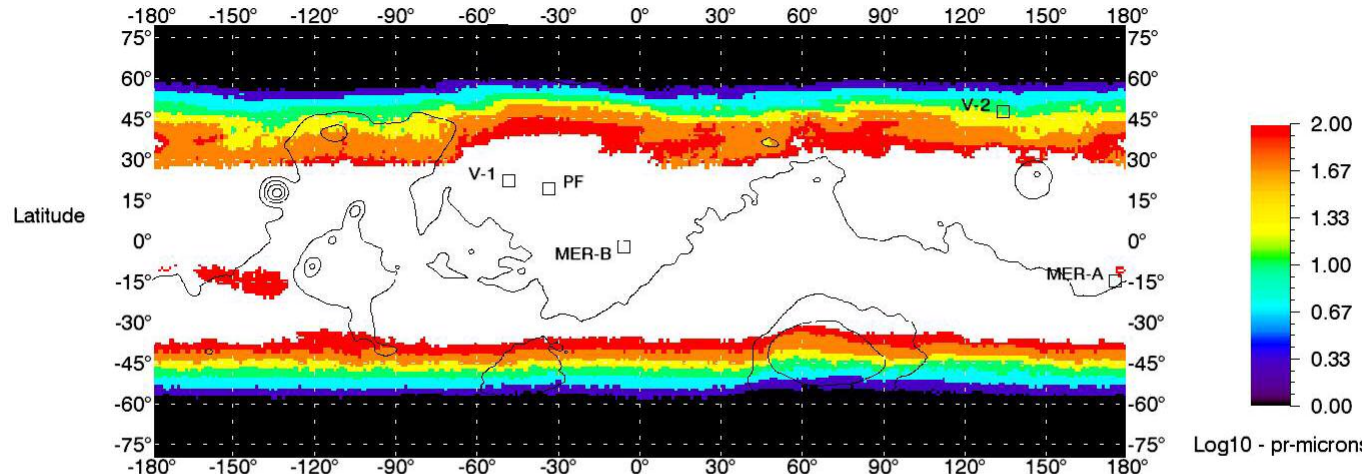

Log10 - pr-microns

Longitude

Figure 31 Variations in the ground-ice distribution as a function of the water content of the atmosphere. The base atmospheric water contents are corrected for elevation and depleted in the near-surface atmosphere. The top panels show the depth to ice for selected water contents. The lowermost panel shows the variation in the extent of ground ice for all water contents on a single map - no depth-to-ice information is shown here. In this lowest panel, ground ice is stable everywhere from the pole to the extent of the color indicated for a particular atmospheric water content. Note that ground ice is never stable at low latitudes with the orbit from the present epoch because the vapor depletion algorithm limits the amount of water the atmosphere can hold here. 
We had initially expected to see the ice extent increase greatly as more water was put into the atmosphere so that ice would extend across low latitudes and the equator in the same way as was shown in Figure 4 of Jakosky et al. (2005). However, our Figure 31 suggests that this does not happen. The distribution of ground ice in our model is much more restricted and never reaches the lowest latitudes, even for the highest atmospheric water content shown, $100 \mathrm{pr}-\mu \mathrm{m}$.

The reason for this is that the water-vapor depletion scheme we use applies a saturation limit to the amount of water that can be held in the atmosphere on any day. Around low northern latitudes with low thermal inertia, the water carrying capacity of the atmosphere is 30 to $40 \mathrm{pr}-\mu \mathrm{m}$, Section 2.2.3.1 describes the derivation of the water carrying capacity. When a water content greater than the capacity limit is applied, the column abundance of water vapor is the saturated limit each day of the year and the vapor density of the near-surface atmosphere is determined based on the saturated column and surface temperatures through each day. Consequently, the effective vapor density ratio for the near-surface atmosphere with respect to the high water content applied is reduced since the atmosphere is never allowed to be super saturated. While there are certainly caveats associated with our simple scheme to find the effective water vapor density of the near-surface atmosphere, as mentioned when the depletion scheme was described in Section 2.2.3, this result does agree with comments of other workers. That is, the observation that in many places the martian atmosphere is close to saturation despite its low absolute water content (e.g., Davies 1979). Imposing atmospheric water contents 
that are greater than the present water carrying capacity of the atmosphere can not be done without other considerations such as ways of increasing this water carrying capacity of the atmosphere for the present epoch.

To help illustrate this point, Figure 32 shows the maximum average atmospheric water content over the martian surface as determined by our model. There is somewhat of a correlation between the maximum water content and the average annual surface temperature (as shown in Figure 29). As expected, warmer parts of Mars are capable of holding more water in the atmosphere. However, the saturation vapor density is essentially an exponential function of temperature, so when averaging the water carrying capacity over a year you get a very different trend than when averaging surface temperatures. During the summer seasons at the poles, both the surface temperature and the water carrying capacity increase. Because the summer season is a fraction of the year, the annual average surface temperatures are still low with respect to low latitudes (for the present obliquity). However the exponential increase in the atmospheric water carrying capacity during summer means that even after averaging over all seasons, the polar atmosphere can hold as much water as low latitudes. Note that there is an asymmetry between the poles. The southern pole experiences summer near perihelion making the southern summer shorter but hotter. Consequently, the southern polar region is colder on average but yet the southern polar atmosphere can hold more water. 


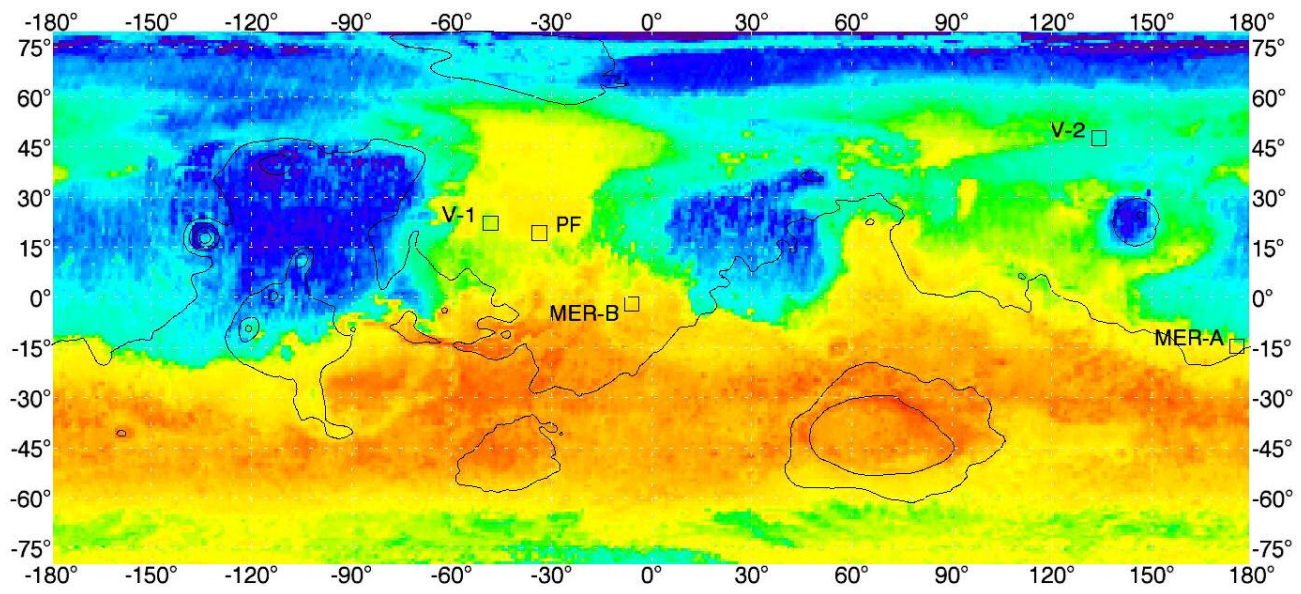

Water carrying capacity - Log10 - pr-microns

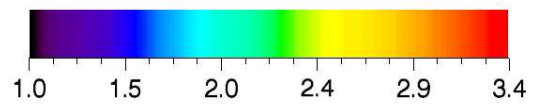

Figure 32 Atmospheric water carrying capacity for Mars in the present epoch as determined with the vapor depletion algorithm. Interestingly, the low-latitude regions with low water carrying capacity coincide with areas where TES observes high water content in the atmosphere. The TES water content here is $\sim 20 \mathrm{pr}-\mu \mathrm{m}$ (Smith 2002), which is still less than the 30 to $40 \mathrm{pr}-\mu \mathrm{m}$ limits shown above.

As has been mentioned before, the martian atmosphere is close to saturation despite having a low absolute water vapor content in some locations. Figure 32 illustrates a caveat to this point. While in much of the northern hemisphere the observed water content is relatively close to the maximum limit calculated here, in the south the atmosphere is very undersaturated. Figure 32 suggests that southern mid-latitude regions have a water carrying capacity 2 orders of magnitude greater than the observed content. This is largely due to high southern temperatures in the local summer.

To further demonstrate the effect of our vapor depletion scheme, Figure 33 was prepared in a similar way to the lowest panel of Figure 31 with varying amounts of water 
in the atmosphere. The difference in Figure 33 is that the vapor depletion mechanism was switched off. The distribution of ground ice shown in Figure 33 closely matches what was shown by Jakosky et al. (2005).

Vapor depletion does not modify the ground ice distribution much when the atmospheric water content is lower than present because depletion is less effective; it takes a larger drop in night time temperatures to deplete the near-surface atmosphere when it is undersaturated. However at water contents greater than present, the nearsurface vapor depletion is significant and reduces the distribution of ice by several degrees of latitude, as seen by the difference in Figure 31 and Figure 33.

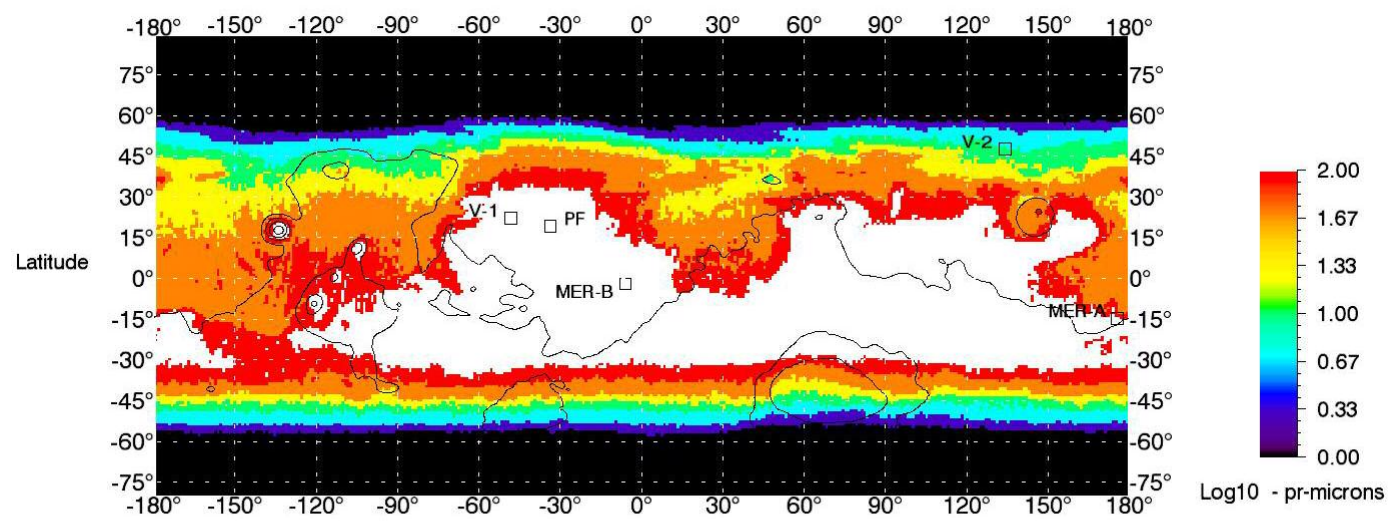

Longitude

Figure 33 Distribution of ground ice if vapor depletion near the surface is not taken into account. Comparing this figure to Figure 31 demonstrates that it is the vapor depletion algorithm of the model that restricts the formation of ground ice at the low latitudes. 


\subsubsection{Orbital Effects}

\subsubsection{Effect of Position of Perihelion}

While Mars has experienced a wide range of obliquities over its history, for the last few hundred thousand years the obliquity has been within a relatively narrow range, within 22 and $28^{\circ}$ while orbit plane of the planet has been at low inclination (Ward 1992). The position of perihelion, or perihelion- $\mathrm{L}_{\mathrm{s}}$, still precesses with a period of $\sim 50$ 000 years while the obliquity has been relatively stable. $L_{s}$ is the solar longitude which is as measure of the angular position of the planet in the orbit; $\mathrm{L}_{\mathrm{s}}=0^{\circ}$ corresponds to when the planet is at vernal equinox. The precession of Mars is enough to change the atmospheric water content as determined by our model and the temperatures of the surface. Figure 34 shows the water content we calculate as a function of perihelion- $\mathrm{L}_{\mathrm{s}}$, with the current obliquity and eccentricity. As described in Section 2.2.5.2, we assume that the amount of water in the atmosphere is determined by the location where surface ice would have the lowest atmospheric water carrying capacity, when averaged over the seasons and corrected to a base elevation. In general, the atmosphere over the pole that experiences summer nearest aphelion has the lowest water carrying capacity. However, the high elevation of the south pole means the vapor density derived here is enhanced slightly when corrected to zero elevation. For the present obliquity, the water carrying capacity of the atmosphere over the poles is less than the carrying capacity over low latitudes; this is not the case at high obliquities. 




Figure 34 Atmospheric water carrying capacity for different perihelion- $\mathrm{L}_{\mathrm{s}}$ with the current eccentricity and obliquity. Shown are the water carrying capacities over each pole by assuming the atmospheric column is always saturated calculated first at zero elevation - dotted lines. The water capacity at each pole is then adjusted (adj.) to an equivalent capacity at a base global elevation; this correction makes the south pole, which is at a higher elevation, able to put more water into the atmosphere. The base water content for Mars at a particular perihelion- $\mathrm{L}_{\mathrm{s}}$ is the minimum corrected carrying capacity of the 2 poles. For the present obliquity, the minimum water carrying capacity is always at one of the poles. 
The thermal model was run for values for perihelion- $\mathrm{L}_{\mathrm{s}}$ of $0,90,180$, and $270^{\circ}$. The results of the ground-ice distribution at these positions are shown in Figure 35 and summarized in Table 4 as models "peri*."

The average surface temperatures are almost identical when perihelion coincides with either equinox (perihelion- $\mathrm{L}_{\mathrm{s}}$ of $0^{\circ}$ or $180^{\circ}$ ) or the orbit with zero eccentricity. This is not surprising because in this case the length and average intensity of the summer in each hemisphere is the same. There is a small variation between the 0 and $180^{\circ}$ models because the hemisphere that enters Spring/Summer at perihelion tends to be warmer. The peak insolation at perihelion is more effective at raising the average temperature if the insolation is subliming seasonal frost than if it is raising the temperature of bare ground hot ground loses more heat in the infrared. 


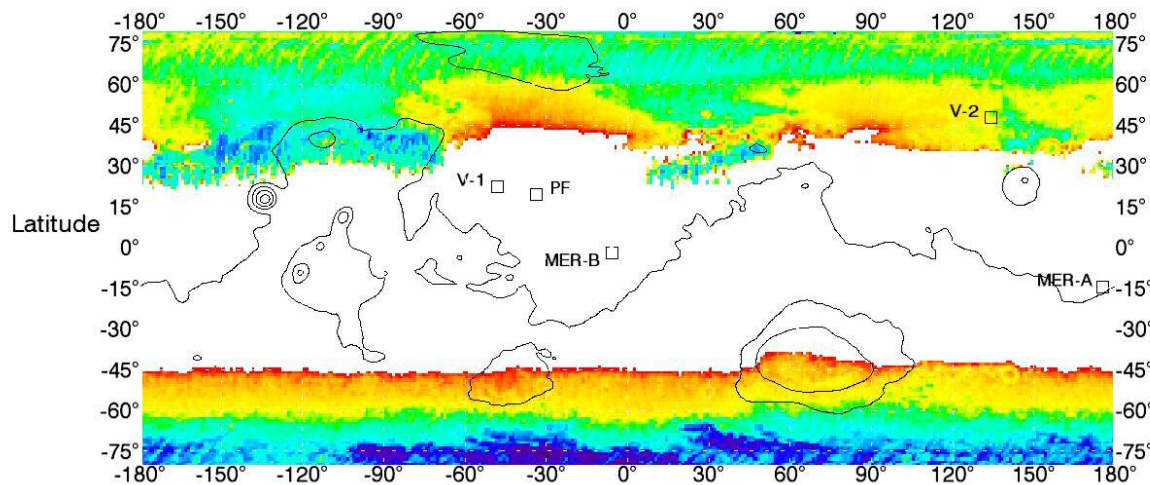

peri180

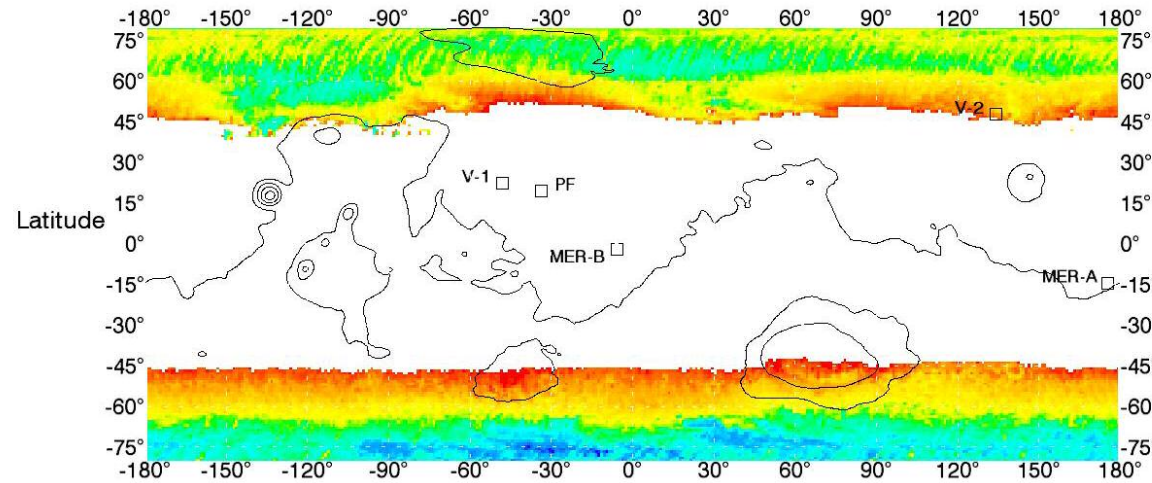

peri270

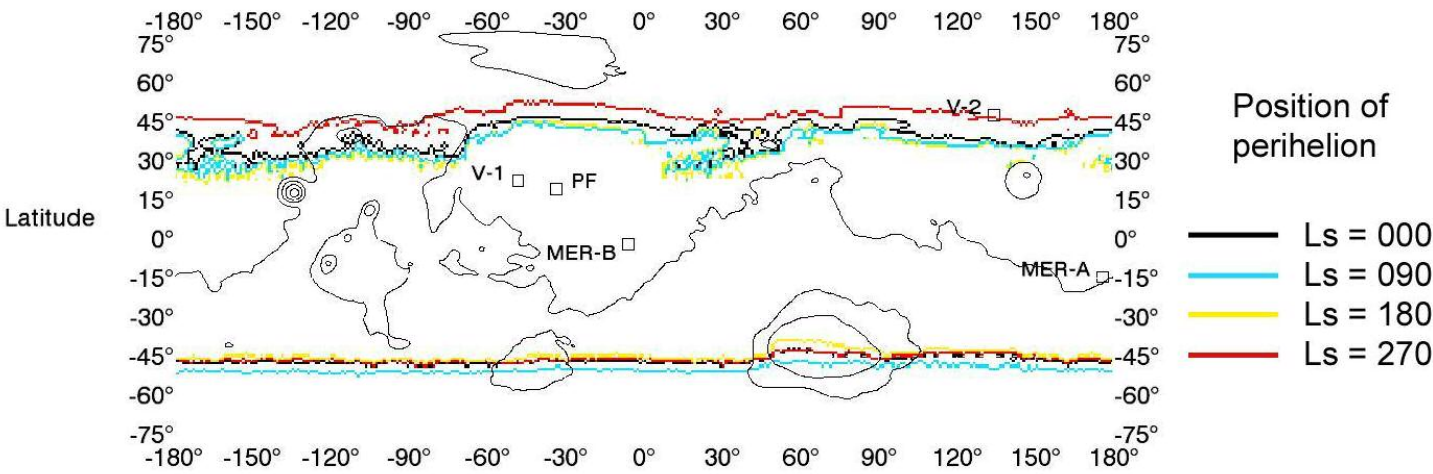

Longitude

Figure 35 The distribution of stable ground ice for different perihelion- $L_{s}$ with the current eccentricity and obliquity. The top two panels show the depth to ground ice for 2 positions of perihelion, 180 and $270^{\circ}$. These perihelion positions correspond to approximately the respective maximum and minimum extent of ground ice in the northern hemisphere. The lowest panel shows the extent of the ground ice for 4 arguments of perihelion tested: $0,90,180$ and $270^{\circ}$. Ground ice is stable from the pole to the line indicated in each hemisphere. 
When perihelion coincides with a solstice (perihelion- $\mathrm{L}_{\mathrm{s}}=90$ or $270^{\circ}$ ) there is a large north-south asymmetry in the length and intensity of the seasons. The hemisphere with the perihelion summer season has a short, intense summer; peak temperatures and derived atmospheric carrying capacities are higher, however annual average temperatures are lower. Recall that it is the location with the lowest corrected water carrying capacity that we use to determine the global water content of the atmosphere at any epoch. The difference in the elevations of the north and south poles create an asymmetry in the response of the base atmospheric water and ground ice to precession. When the south experiences a perihelion summer, like the present conditions, the atmospheric water is derived from the north and is low (due to the lower elevation), but the average surface temperatures are also cooler in the south. Both of these processes affect the extent of ground ice, though they work in opposite directions and almost cancel each other out. On the other hand, the northern hemisphere experiences a perihelion summer and lower surface temperatures when there is more water in the atmosphere (coming from the south pole) and both of these processes both increase the extent of ground ice in the north. Consequently, there is a lot more variation in the ice extent in the north than in the south, as in Figure 35. The extent of ground ice in the south is always within a few degrees of latitude of the average extent while in the north it varies by more than $10^{\circ}$ over the perihelion cycle.

The present ice extent in the north is near its minimum in the perihelion cycle, though our model indicates that northern ground ice should have been much more extensive, by 10 to $15^{\circ}$ in latitude, at perihelion $-\mathrm{L}_{\mathrm{s}}=180^{\circ}$ which was $\sim 10$ thousand years 
ago. Jakosky et al. (2005) has already suggested there may have been more water in atmosphere in a recent epoch and this may have deposited ice at low latitudes, note though that the extent of ground ice we find at perihelion- $\mathrm{L}_{\mathrm{s}}=180^{\circ}$ is still substantially different from the high hydrogen abundances inferred with neutron fluxes (Feldman et al. 2002).

It may take a long time for ground ice to ever reach the 'stable' distribution calculated with thermal models, whether by deposition or sublimation. However, it would be hard for remnant ice to remain very close to the surface and be detectable by remote sensing when it has been unstable there for several thousand years; an impermeable layer, essentially a piece of plastic, would be required to isolate ice from the atmosphere. Hydrated minerals and salts are more likely candidates for the high hydrogen contents observed at low latitudes (Feldman et al. 2004a). That point aside, there are regions of high averaged atmospheric water content observed by TES (Smith 2002) that roughly coincide with the longitudes where northern ground ice extended furthest in the recent past in our models. These longitudes are also the regions of low thermal inertia. Deeper reservoirs of ice below the sensitivity of remote sensing in the process of slowly subliming are not necessarily ruled out as a source of groundatmosphere exchange playing a role in the high atmospheric water of these regions.

We demonstrate that the perihelion- $\mathrm{L}_{\mathrm{s}}$ can drive some minor variations in the ground-ice distribution, especially in the north. This may modify ground ice during epochs like the present when obliquity is relatively stable over few thousand years. We 
will compare the contribution of perihelion- $\mathrm{L}_{\mathrm{s}}$ changes to the small obliquity changes at the end of the following section.

\subsubsection{Effect of Obliquity}

As mentioned above, the obliquity of Mars has been within a relatively narrow range over the last few hundred thousand years (between 22 and $28^{\circ}$ ). Beyond this time range, the obliquity has varied from 15 to $35^{\circ}$ in the last few million years in oscillations with a period of 120000 years. The amplitude of these 120000 year oscillations are modulated in a cycle of the order of 1 million years. Several models of the past obliquity of Mars (e.g., Laskar et al. 2004) now show that there was a time beyond 5 million years ago that the obliquity was higher and oscillated from $30^{\circ}$ to $45^{\circ}$. Further back in time martian obliquity varied more, though it is harder to model.

These large changes in obliquity have long been recognized to have potential to drive large changes in the martian climate in response to a different insolation distribution over the latitudes (e.g., Ward 1992). Recently global circulation models have been run at different obliquities, giving us some idea of the climate and water cycle at these times (Mischna et al. 2003; Mischna and Richardson 2005). Here we explore the response of ground ice to obliquity. Our intention here is to see how the ground ice varies as a function of obliquity. Now we want to suppress the effects of eccentricity and perihelion- $\mathrm{L}_{\mathrm{s}}$. To do this we set the eccentricity to zero. We found that when eccentricity is zero for the current obliquity of Mars this increases the atmospheric water 
content by about a factor of 2 because Mars is closer to the Sun during the northern summer which raises the summer temperatures of the north pole.

As discussed in the Section 2.2.5.1, we allow the pressure of the atmosphere to vary as a function of obliquity. For obliquities lower than present, we use an energy balance at the poles to estimate the atmospheric pressure - this idea is based on the principle that the atmosphere responds to a permanent $\mathrm{CO}_{2}$ cap at one pole or the other. For higher obliquities we use the present pressure, as shown in Figure 36.

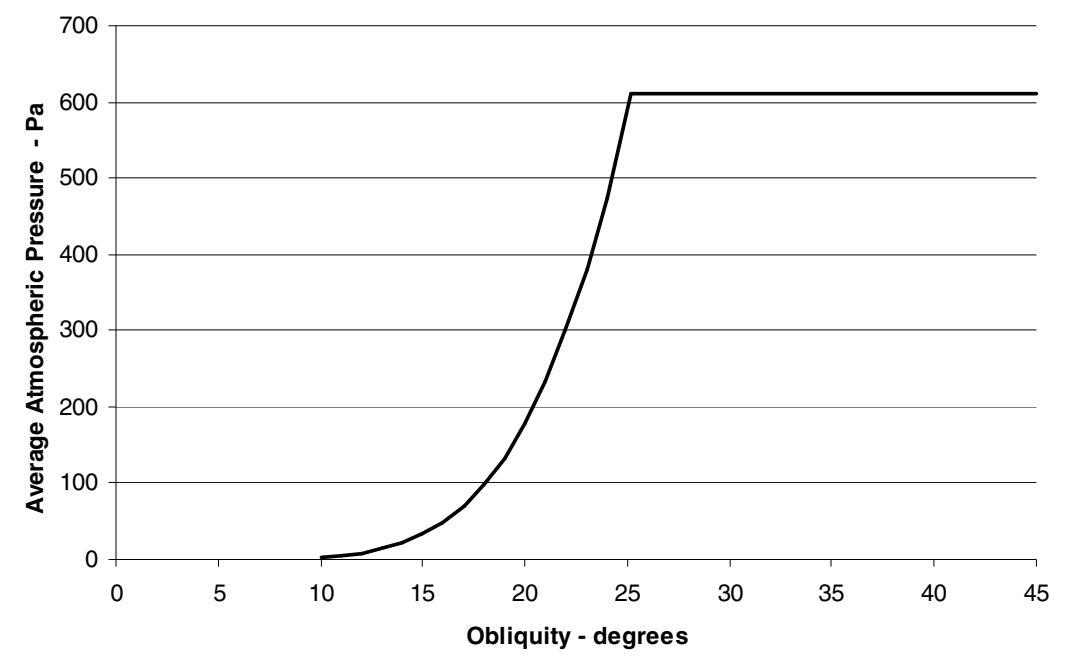

Figure $36 \mathrm{CO}_{2}$ as a function of obliquity, as used for this project.

The atmospheric water content at different obliquities is estimated by finding the minimum in the water carrying capacity (or the saturated water content) of the atmosphere over surface ice at different latitudes, as discussed in Section 2.2.5.2. Figure 
37 shows the average atmospheric water content as a function of obliquity that we use. The water content estimated previously (Jakosky et al. 1993; Mellon and Jakosky 1995) is also shown for reference in Figure 37. It can be seen that there is an order of magnitude difference between the different approaches at obliquities of around $35^{\circ}$ and more; these obliquities were achieved by Mars as recently as several hundred thousand years ago (Ward 1992; Laskar et al. 2004). We allow the atmospheric water content to be determined wherever the water carrying capacity of the atmosphere is a minimum, which is outside the polar regions for obliquities greater than $30^{\circ}$, see Figure 38 . Jakosky et al.(1993) determine water content from polar regions for all epochs which puts more water into the atmosphere than non-polar regions. For obliquities less than $30^{\circ}$, our variation of water with obliquity is similar to that which was used by Mellon and Jakosky (1995). Our approach uses saturation vapor densities while Mellon and Jakosky (1995) used sublimation rates. While these are different physical processes to estimate water content, the dependences on temperature for saturation density and sublimation rate are similar. Hence both approaches lead to similar atmospheric water contents as functions of obliquity. 


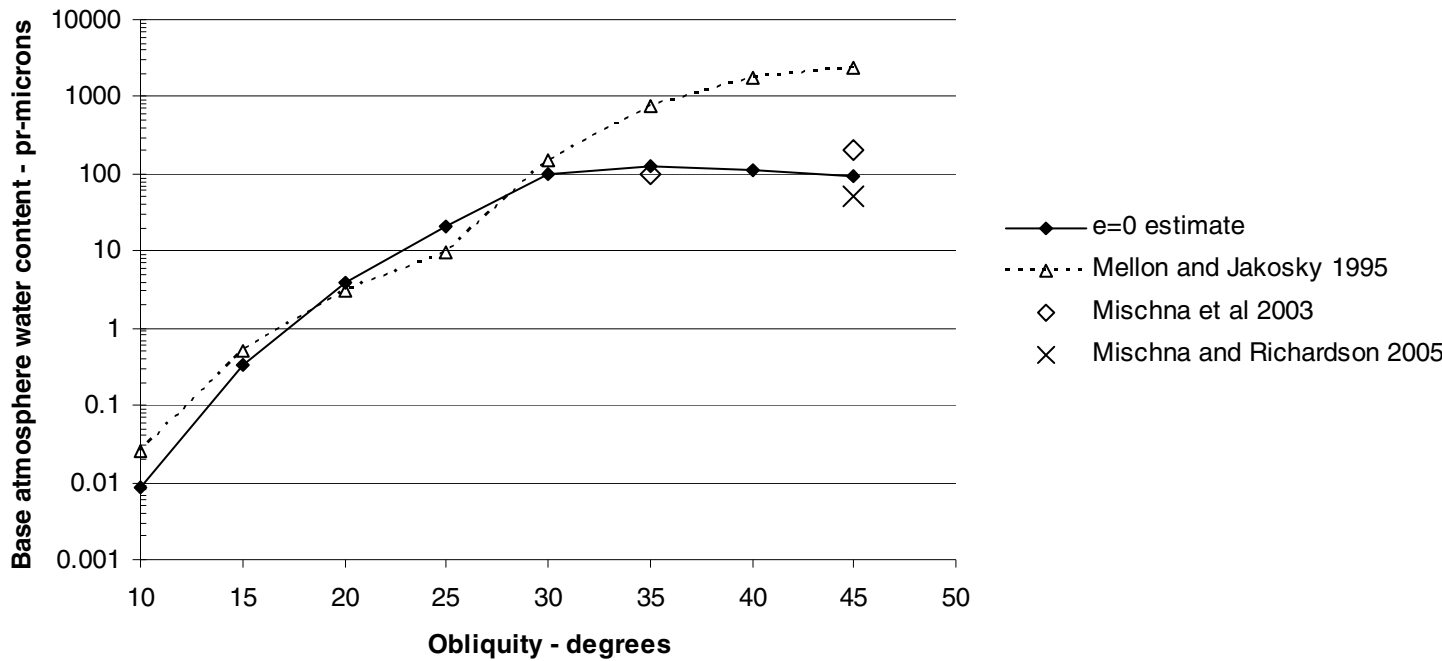

Figure 37 Base atmospheric water content as a function of obliquity. Also shown are approximations that have been used in the literature. The dotted line is based on equations given in Mellon and Jakosky (1995) which use the seasonal sublimation from ice at the poles to estimate the water vapor in the atmosphere. Mischna et al. (2003) ran a GCM at high obliquities and points shown indicate the approximate water vapor content found with their model. The GCM used by Mischna and Richardson (2005) has a lower water content because in this model the polar ice is inactive.

Also shown in the Figure 37 are average atmospheric water contents from a GCM at obliquities of 35 and $45^{\circ}$. In the one scenario a residual water cap is exposed and readily able to supply water to the atmosphere (Mischna et al. 2003). In another scenario the polar residual cap is isolated from the atmosphere, either by removal of the cap or insulation by a lag deposit (Mischna and Richardson 2005). The atmospheric water content is then determined by surface ice deposits at low latitudes which give a water content much less than if surface ice were exposed at the poles. These GCM results are 
consistent with our observation that at low obliquities the poles have the lowest water carrying capacity, while at high obliquities this occurs at low latitudes, as shown in Figure 38. We assume these locations with a minimum water carrying capacity are preferred locations for surface ice to build up. These GCM results also indicate that our simple approach to determining water content used here gives a reasonable estimate of the water response to obliquity.

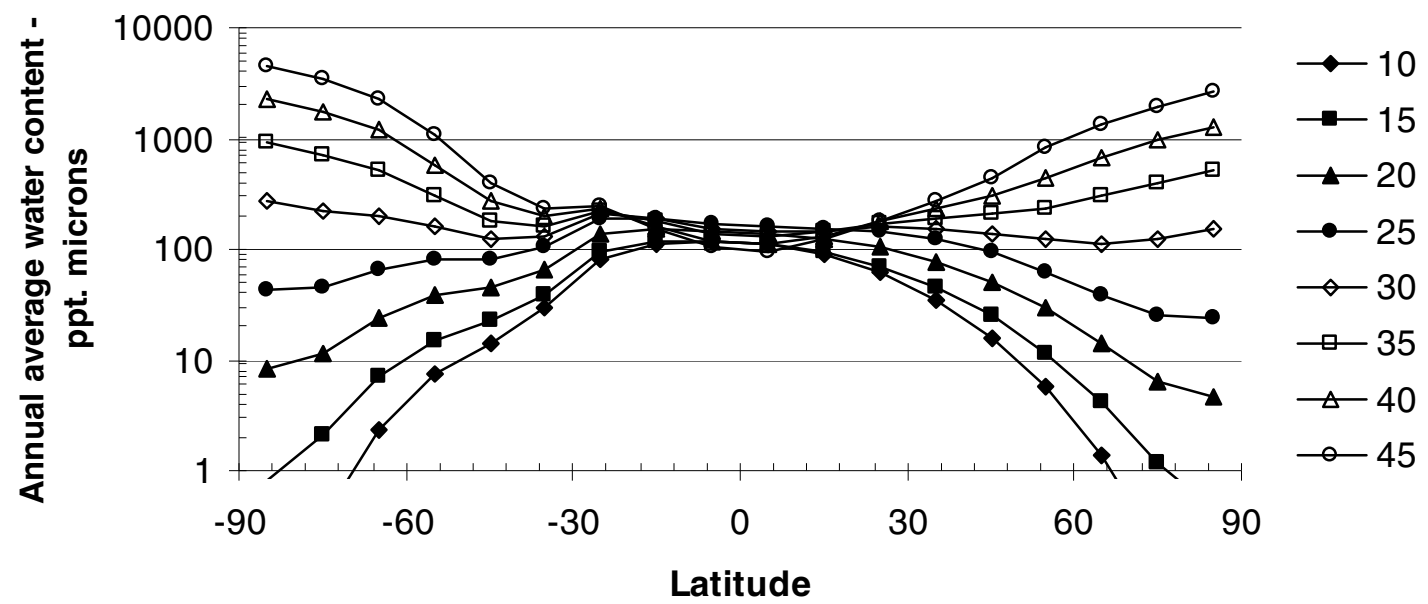

Figure 38 Atmospheric water-carrying capacity over exposed surface ice as a function of latitude and obliquity. For this plot, the eccentricity of the planet's orbit is zero and the water content shown is adjusted for the lowest elevation of the ground at this latitude band of Mars. Note that at obliquities greater than $3^{\circ}$ it is at low latitudes where the atmosphere can hold the least water. At low obliquities such as the present orbit, one of the polar regions represents a minimum in atmospheric water. Note also that when eccentricity is non-zero, this introduces a north-south asymmetry that is not represented in this plot here.

Note that at the lowest obliquities, $\mathrm{CO}_{2}$ will no longer be the dominant component of the atmosphere. Nitrogen and argon are present in the atmosphere and have partial pressures of 17 and $10 \mathrm{~Pa}$ respectively. The average partial pressure of $\mathrm{CO}_{2}$ we estimate 
at $15^{\circ}$ obliquity is comparable to the sum of these components. When $\mathrm{CO}_{2}$ becomes a minor component of the atmosphere it is likely to have implications for the climate at these epochs. For example, how effective will the $\mathrm{CO}_{2}$ be at buffering the surface temperature at the frost point? This has potential implications to thermal models at these epochs as well that we do not consider here. The atmosphere is depleted at these epochs, much thinner than the present epoch. The thin atmosphere will have an even smaller impact on the energy balance and temperatures at the surface, so any effects of the atmosphere on surface thermal models will be only minor when obliquity is low.

Thermal models have been run at obliquities of 10 to $45^{\circ}$. Figure 39 shows both the ice depth and distribution at selected obliquities and the lowest panel shows the ground ice extent of all models on a single panel for comparison. 

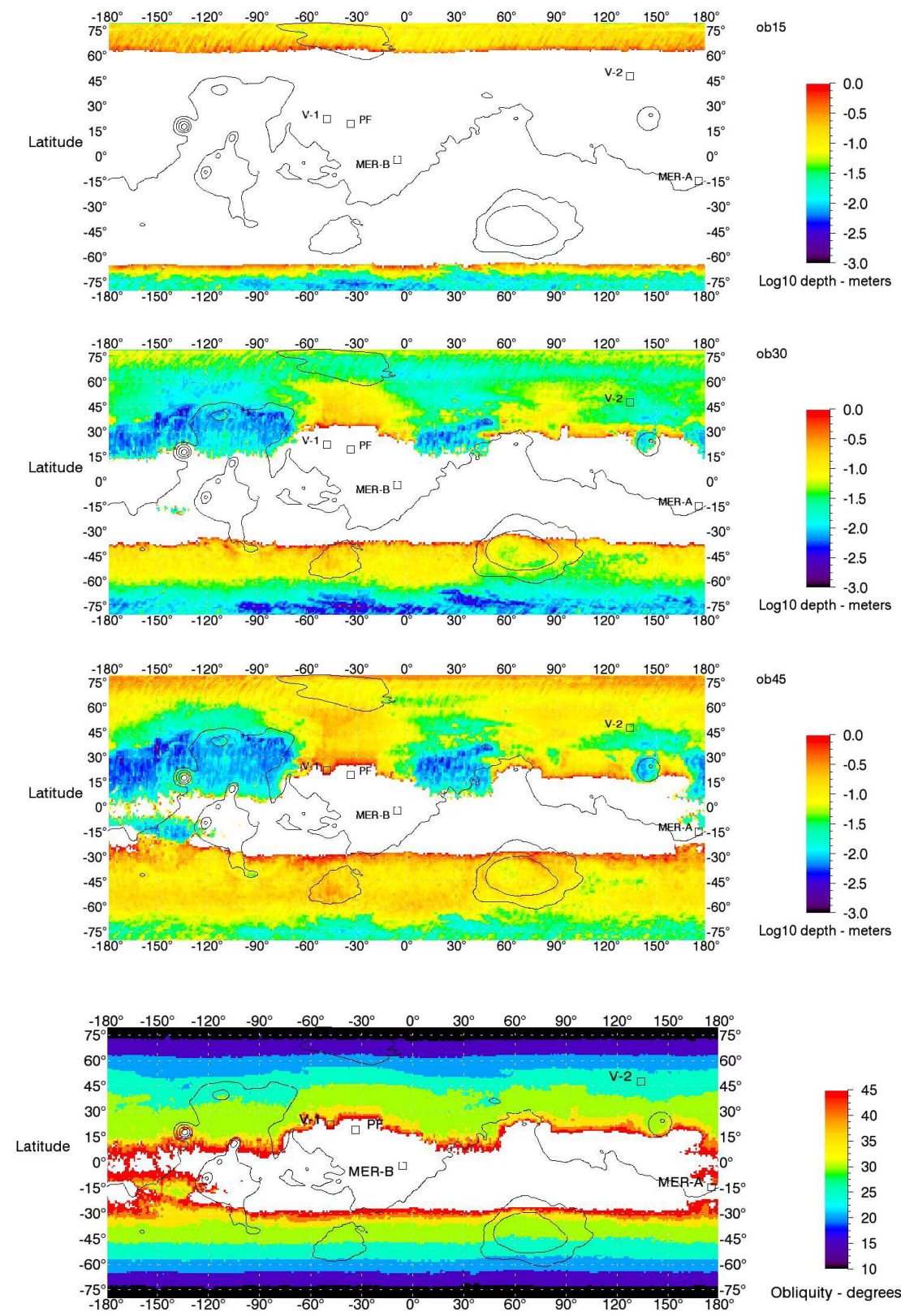

Longitude

Figure 39 The distribution of ground ice for different obliquities with zero eccentricity. The top panels show the ground-ice distribution at indicated obliquities, including the depth to the ice. The lowest panel shows the variation in just the extent of the ground ice for all the obliquities calculated. Atmospheric pressure and atmospheric water density have been allowed to vary as a function of obliquity, as described in the text. Note that ground ice never becomes stable globally. 
Mellon and Jakosky (1995) was the last paper to address the question of where ground ice would be stable at past epochs. They indicated that ground ice could become stable everywhere on Mars at obliquities as low as $32^{\circ}$. We find that this is not the case, even at the highest obliquities we test. We find ground ice is never stable at low latitudes with the exception of longitudes 120 to $180^{\circ} \mathrm{W}$, just west of Tharsis, which is where a region of low thermal inertia crosses over the equator. Outside this region, the approximate limiting latitudes of stable ground ice are $\sim 25^{\circ} \mathrm{S}$ and $\sim 15^{\circ} \mathrm{N}$. Our lower extent of ground ice at high obliquities is partly due to the near-surface depletion of the water vapor as well as the lower global water contents we estimate at these high obliquities. Ground-ice extent does increase monotonically with obliquity, despite a decrease in the water content of the atmosphere for obliquities greater than $35^{\circ}$. However, the rate of increase in ground-ice extent with obliquity drops notably when obliquity is greater than $30^{\circ}$. Note that while ground ice is more extensive at high obliquities, the depth to stable ground ice at high latitudes increases. Ice is still stable at these high latitudes at high obliquity, though average temperatures increase here with obliquity which increases the depth to stable ice. In the ice depth maps at high obliquity, the low thermal inertia regions show clearly as broad regions of shallow, stable ground ice. Thermal skin depths are smaller in regions of low thermal inertia which makes ice stable closer to the surface.

In order to quantify the contribution of the lower atmospheric water content (with respect to water contents used by Mellon and Jakosky, 1995) and near-surface vapor 
depletion to the reduction of extent of ground ice at high obliquities, some extra maps were generated to isolate the effects of these components. Table 5 shows a summary of these maps; the obliquity of all these models is $35^{\circ}$ and eccentricity is zero. In models with “...high_h2o...," we apply a higher atmospheric water content based on the water content used by Mellon and Jakosky (1995). In models with “...no_vap_dep...,” the near-surface vapor depletion algorithm is switched off. In one model we apply high atmospheric water content and no vapor depletion and find the ground ice becomes globally stable, as found by Mellon and Jakosky (1995). Results in Table 5 demonstrate that vapor depletion is more effective at reducing the extent of ground ice than the lower atmospheric water we use. However, at high latitudes, the lower water content we use is more effective at increasing the depth at which ice is stable.

\begin{tabular}{|c|c|c|c|c|c|c|c|}
\hline Map & $\begin{array}{l}\text { Atmo. } \\
\text { H2O - } \\
\text { pr- } \mu \mathrm{m}\end{array}$ & $\begin{array}{l}\text { Av. } \\
\text { Sth. } \\
\text { Limit }\end{array}$ & $\begin{array}{l}\text { Av. } \\
\text { Nth. } \\
\text { Limit }\end{array}$ & $\begin{array}{l}\text { Depth } \\
70.5 S\end{array}$ & $\begin{array}{l}\text { Depth } \\
60.5 S\end{array}$ & $\begin{array}{l}\text { Depth } \\
60.5 \mathrm{~N}\end{array}$ & $\begin{array}{l}\text { Depth } \\
70.5 \mathrm{~N}\end{array}$ \\
\hline ob35 & 128 & -30.2 & 19.3 & 1.73 & 5.59 & 4.45 & 5.29 \\
\hline ob35_high_h2o & 780 & -13.3 & 14.3 & 0.61 & 1.86 & 1.40 & 1.33 \\
\hline ob35_no_vap_dep & 128 & -12.6 & 5.1 & 1.59 & 4.63 & 3.50 & 4.76 \\
\hline ob35_high_h2o/no_vap_dep & 780 & no limit & no limit & 0.39 & 0.93 & 0.27 & 0.18 \\
\hline
\end{tabular}

Table 5 Summary of ground-ice distribution for different models at high obliquity to isolate effects of low atmospheric water content and near-surface vapor depletion. The format of the columns is the same as Table 4.

Figure 38 shows that using our scheme, we expect ice on the surface to be stable at low latitudes at the expense of the polar caps when obliquity is high; note that we consider ice on the surface different to ice in the ground. Meanwhile, Figure 39 shows only a limited extent of ground ice that is stable at these low latitudes at these obliquities. 
So where are these surficial ice deposits and why don't they show up on the ground ice map?

The answer to the first part of that question is that in our scheme used here, we assume surface ice forms in the lowest elevations of any latitude band. The reason for this is that we place ice on the surface where the atmosphere above it has the lowest water carrying capacity when corrected to a base level. This correction to a base level decreases the equivalent carrying capacity of low elevations relative to high elevations. These punitive deposits of surface ice at low-elevation, low-latitude sites may yet be associated with some of the sedimentary layers identified at these locations (Malin et al. 2001); it has been noted before that there is a striking similarity between the layered deposits at low latitudes with those at the poles (Leovy et al. 2004). Speculatively, some of these layered deposits have been and are still being constructed episodically during times of moderately high obliquity as recently as a few million years ago. We do not account for any atmospheric circulation in our model; circulation may favor highelevation sites for the precipitation of water onto the surface. Evidence for the formation of high-elevation ice deposits at low latitudes on the western flanks of the Tharsis Montes comes from initial results of high obliquity GCMs (Haberle 2004) and interpretations of geomorphology (Lucchitta 1981; Head and Marchant 2003).

The second part of the question is a little more intriguing. If ice is going to be stable on the surface at a particular latitude, one might think that ice should be stable in the ground there as well, but yet that is not shown in Figure 39. In short, the explanation is that different processes are controlling the stability of surface ice and ground ice. 
Surface ice is presumably precipitated direct from the atmosphere (a process beyond the scope of our models to handle in detail) while ground ice is stable when there is zero net vapor diffusion between the ground ice and the local, near-surface atmosphere. To illustrate with a perhaps extreme example, on the Earth you do not always find stable ground ice or permafrost adjacent to places where glaciers are found. In more detail, the answer to this discrepancy has to do with the relation between the ice and the water vapor in the atmosphere, at least in the way our scheme works. Though ice may be present on the surface at one latitude and freely saturating the near-surface atmosphere above it, ground nearby at the same latitude will not necessarily be in communication with this same saturated air. Instead, the ability of the atmosphere to transport water vapor away from surface ice is limited to the carrying capacity of the atmospheric column above it. The atmospheric water carrying capacity is related to the saturated water vapor density of average diurnal temperature of the ground (as described in Section 2.2.3), so the equivalent surface vapor density of the atmospheric column will be less than the averaged vapor density over the surface ice. As this saturated column is carried away from the surface ice, it is able to supply water to the near-surface atmosphere over adjacent, icefree ground. However, due to the vapor depletion process of the near-surface, the average vapor density of the near-surface atmosphere over adjacent ice-free ground will be significantly less than the average density that was over the surface ice. This means that ice on the surface at one location may be incapable of supplying sufficient water into the atmosphere above to make ice stable at any depth in ground just adjacent to it. 
How, then, will this surface ice form in the first place? In our scheme we consider that ice precipitated directly from the atmosphere. And it is because these locations represent a minimum in the atmospheric water carrying capacity that ice forms there.

Note that in Figure 38, where we show the latitudes where surficial ice would build up, results tend to be symmetric about the equator with the exception of some topographic effects. This would seem to imply the equator or nearby latitudes are the preferred locations for surface ice at high obliquities. What should be remembered is that these results were produced with zero eccentricity to avoid asymmetries related to eccentricity and the perihelion- $\mathrm{L}_{\mathrm{s}}$. In reality, the eccentricity of Mars has rarely been close to zero, the historical average is $\sim 0.07$ (Laskar et al. 2004). Eccentricity-related asymmetries in the climate during epochs of high obliquity will favor surficial ground ice at a range of low latitudes rather than just near the equator as may be suggested in Figure 38.

We have now presented the response of ground ice to the perihelion- $\mathrm{L}_{\mathrm{s}}$ and obliquity and we are in a position to compare the response of the ground ice to changes in these two components. This has implications for the present epoch and the last few hundred thousand years when the variations in the obliquity have been small $\left(\sim 5^{\circ}\right)$ relative to amplitude of the $\sim 120000$ year oscillations over the history of Mars. In the long term, ground ice changes in response to the wide range of martian obliquity will be more significant than changes due to perihelion. In Section 2.3.2.1 we found the variation in the extent of ground ice was $\sim 6^{\circ}$ in the south and $\sim 14^{\circ}$ in the north in 
response to the perihelion cycle. The ground ice extent results shown in this Section indicate that a change in obliquity of $5^{\circ}$ in the present epoch would change the extent of ground ice by $\sim 10^{\circ}$ in the south and $\sim 15^{\circ}$ in the north. From this we conclude both the position of perihelion and obliquity will have had made similar contributions to changes in the ground ice extent in the last few hundred thousand years of Mars. Ground ice in the south is more sensitive to obliquity than the position of perihelion by a factor of 2 , though in the north the contributions are about equal.

\subsection{Discussion}

In this section we discuss some implications of our results presented above with respect to features observed on the surface of Mars. Ground ice should play a significant role in the morphology of the surface of Mars; for example: polygons, rock glaciers, mantled surfaces, creeping ground, linear valley fill (e.g., Squyres and Carr 1986). The distribution of ground ice is demonstrated to be a sensitive function of climate. We have demonstrated how climate change driven by orbit evolution and a few simple processes can change the ground-ice distribution significantly in recent geological history. Consequently, many surface features observed, except the most recent features, have almost certainly formed in conditions that are different to the present martian climate. Here we will discuss the distribution of two types of landforms with respect the extent of martian ground ice in past epochs: dissected mantles and terrain softening. 
Mantle cover showing varying degrees of dissection in the mid latitudes was first mapped out by Mustard et al. (2001). This terrain was then divided by Milliken and Mustard (2003) based on the surface texture. Localized removal, knobby, wavy and scalloped textures were identified; samples of these textures are shown in Figure 40. The extent of these dissected mantles has been attributed to the extent of deposition of ice and dust from the atmosphere during periods of high obliquity and later desiccation during low-obliquity epochs. A map showing the distribution of these terrains is in Figure 41. Possibly related to these mantles, GCMs by Lavrard et al. (2004), suggest that the high water content deposits at higher latitudes $\left(>60^{\circ}\right)$ probably formed at epochs of low obliquity while surface ice at low latitudes is still left from a previous epoch of high obliquity. 

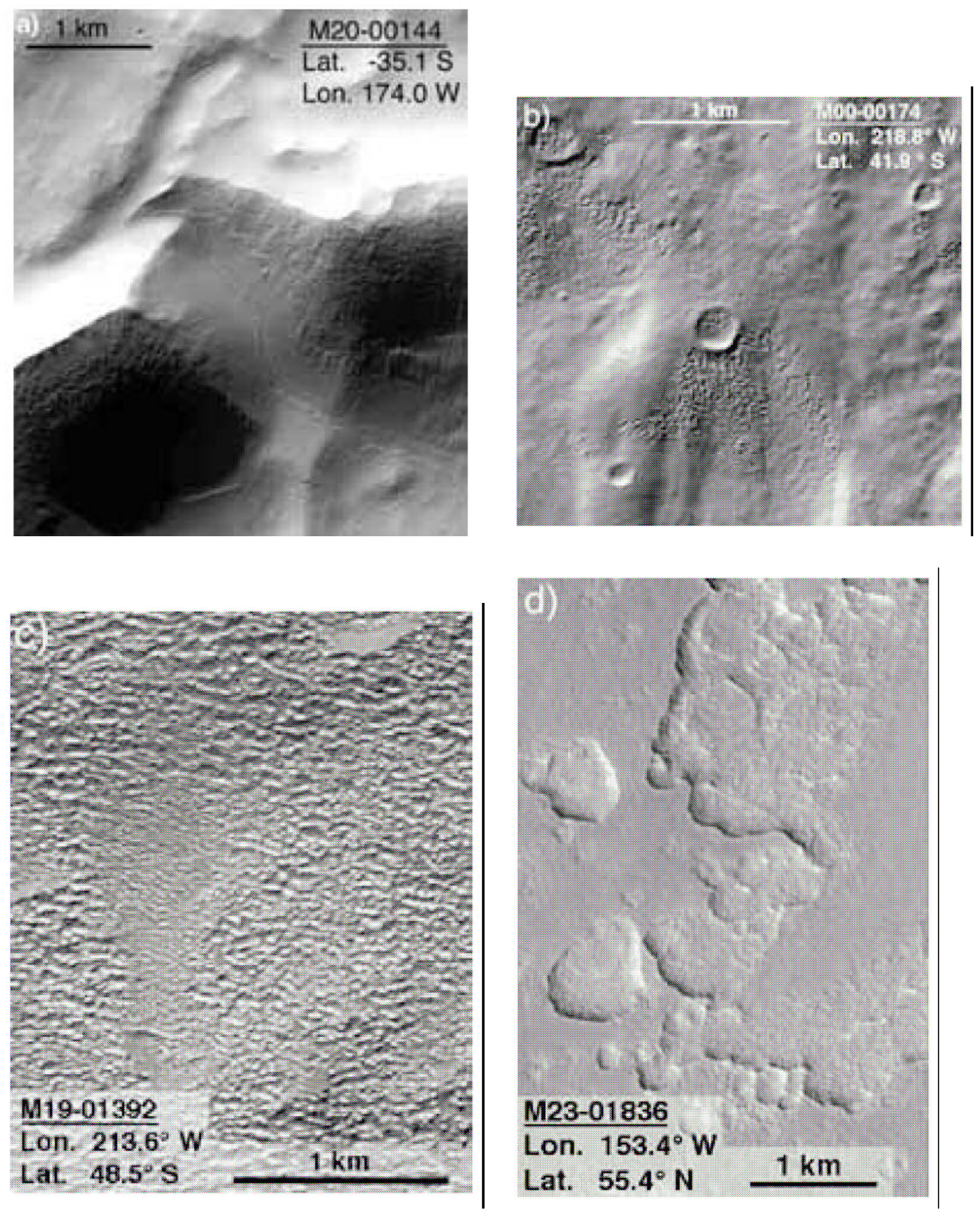

Figure 40 Images from Milliken and Mustard (2003) showing various styles of dissected mantle mapped. Styles of dissections are: a) localized complete removal, b) knobby texture, c) wavy texture, and, d) scalloped and total mantle cover. 


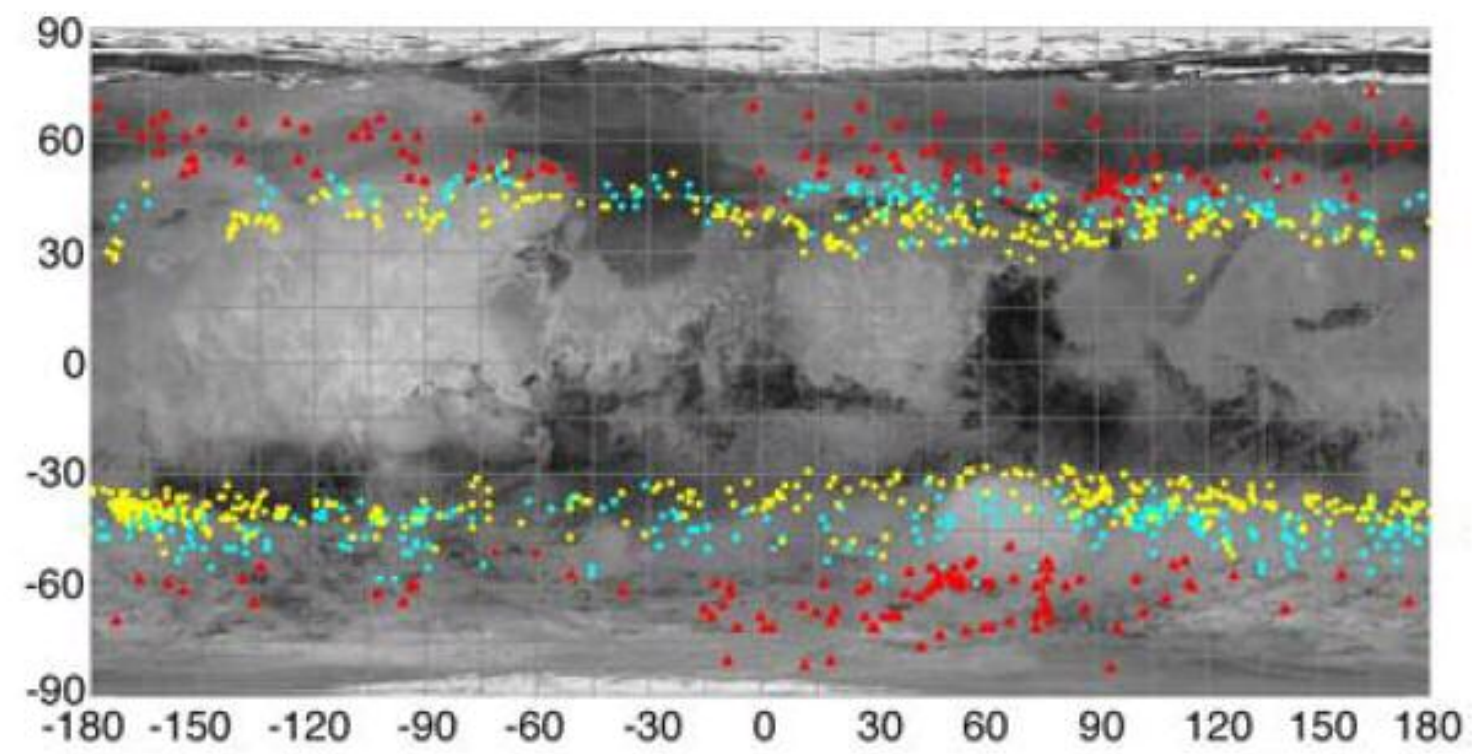

Figure 41 Map from Milliken and Mustard (2003) showing the distribution of various dissected mantle textures. Textures are based on a survey of MOC images. Yellow dots indicate localized complete removal; blue indicates knobby and wavy textures; red indicates scalloped and total mantle cover.

The thickness of this mantle in places where it is dissected is of the order of several to tens of meters thick. It may even be thicker at high latitudes where mantle cover is complete and there is no dissection. Such dissections of the mantle imply significant volume loss and a high ice content in the original mantle material. The probable way to form this thick, water-rich mantle cover is for large, rapid swings in the obliquity to expose surface ice to high insolation, either low-latitude ice at low obliquities or polar ice at high obliquities. The ranges of latitude showing localized and knobby dissection roughly coincide with the range of limits to stable ground ice induced by the position of perihelion (Figure 35). However, the position of the perihelion with the 
present obliquity is not able to expose any surface ice to high insolation and is unlikely to form the mantle in the thickness and high water content that is observed. Hence it is also unlikely that precession of Mars and changes in the position of perihelion are capable of driving the formation of significant mantle deposits.

Maps of ground-ice stability indicate where ice-rich mantle deposits are unstable and most susceptible to dissection. In general, ice is never stable when exposed on the surface, even where ground ice is stable. However, sublimation rates may be less than deposition rates and this may allow ice, or an ice-dust mix, to build up and form a mantle cover. We do not try to estimate deposition rates in our models. If an ice-dust mix is present on the surface where ground ice is stable, the build up of ice-free material at the surface, or lag, can slow the sublimation and ultimately allow ice-rich mantle to be stable for a period. If obliquity changes so that ground ice is no longer stable, no amount of lag deposit is able to protect ground ice (assuming the lag is permeable) and the ice-rich mantle will desiccate.

The low-latitude limit of the mantle deposits is related to either the limit of the mantle deposition during high-obliquity epochs or the limit to which low-obliquity epochs have been able to completely deplete the mantle. Topography is likely to create local pockets where ice-rich mantle deposits can be stable at low latitudes even during low-obliquity epochs; this effect is something that is not included in models presented here. Indeed, the style of mantle dissection found at lowest latitudes, "localized complete removal" from Milliken and Mustard (2003), shows mantle restricted to topographically favorable locations. 
The high-latitude limit of the knobby and wavy units of the dissected mantle, as defined by Milliken and Mustard (2003), coincides with our limit of stable ground ice in the present epoch. The style of dissection of the mantle locations at higher latitudes where ground is stable at present are the scallop features (Milliken and Mustard 2003). At these high latitudes, a thin lag over these scallop features would be sufficient to make ground ice stable to diffusion; so these features should be stable at present. These scallop features should have formed during low-obliquity epochs when the ice in the mantle was unstable. If this is the case, the scallops may have formed relatively quickly as Mars has only had relatively brief periods of low obliquity in the last few million years. The poleward limit of the scalloped-mantle terrain is $65^{\circ} \mathrm{N}$ and $\mathrm{S}$, which coincides nicely with the limit of ground ice at an obliquity of $15^{\circ}$; see histogram in Figure 42 . An obliquity of $15^{\circ}$ is the lowest obliquity Mars has experienced in the last few million years. 


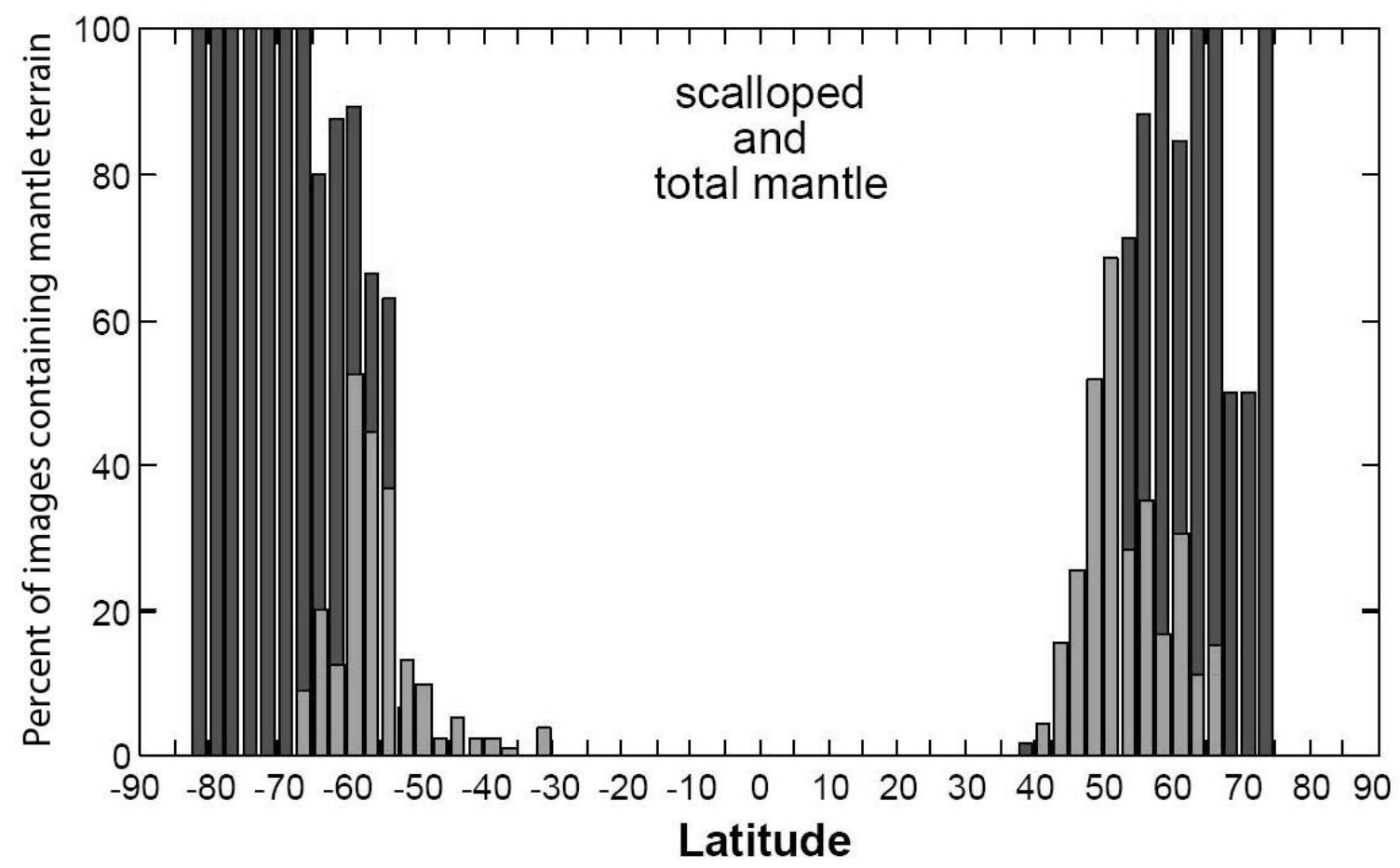

Figure 42 Histogram from Milliken and Mustard (2003) showing the distribution of scalloped (grey) and total mantle cover (black). Scalloped mantle is not found beyond $65^{\circ} \mathrm{N}$ or $\mathrm{S}$, which coincides with the extent of stable ground ice at an obliquity of $\sim 15^{\circ}$. 
Another example of landforms potentially related to the presence of ground ice is terrain softening. The extent of terrain softening is common in the mid latitudes while it is absent in low latitudes. Examples of terrain softening are shown in Figure 43. Where softening is present, crater rims are broader and rounded and small angular features, including small craters, are rare. Where softening is absent, crater rims are sharper, the terrain maintains a blocky, angular texture and small craters are more common (Squyres and Carr 1986). While the exact mechanism for the softening may be uncertain, whether it is due to viscous relaxation of topography or burial by mantles, the extent of terrain softening is assumed to be related to the distribution of ground ice (Squyres and Carr 1986).

Previous models of the distribution of ground ice for past epochs (Mellon and Jakosky 1995) have suggested that ground ice would be globally stable at obliquities as low as $32^{\circ}$. Recent dynamic models of the orbit and the obliquity of Mars now suggest that high obliquity would have been the norm, rather than just brief epochs, over of the history of the planet (Laskar et al. 2004). The lack of terrain softening at low latitudes is then inconsistent the theory that ground ice would be global at high obliquity since Mars would have spent much of its history is this state.

In this work we propose that ground ice does not become globally stable, even at obliquities as high as $45^{\circ}$. The difference in our models is a lower atmospheric water content for these high-obliquity epochs and the depletion of water vapor of the nearsurface atmosphere. This lack of global ground ice thus reconciles the lack of terrain 
softening and evidence for ground ice at low latitudes despite long periods of time at high obliquities.
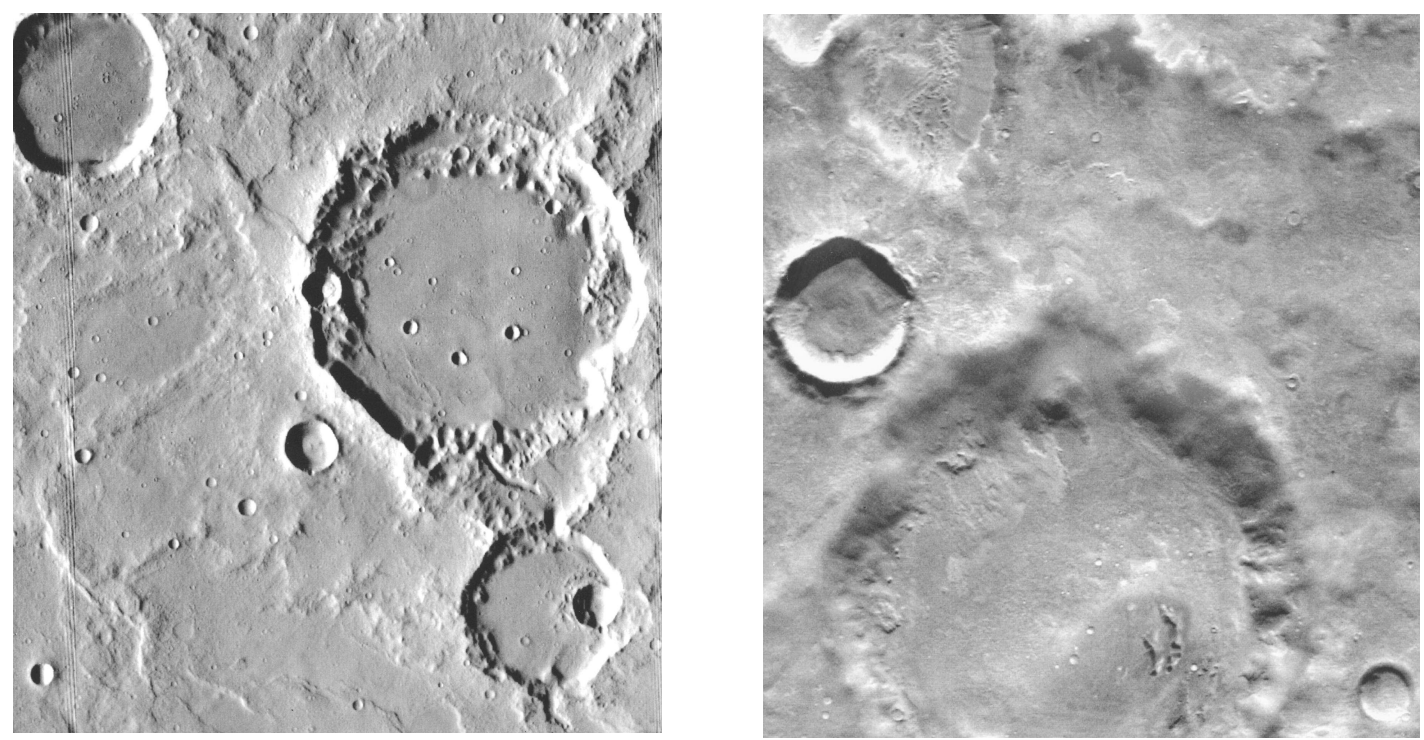

Figure 43 Sample of Viking Orbiter images from low and mid latitudes showing varying degrees of terrain softening. The width of each of the images is $\sim 50 \mathrm{~km}$. The image on the left $(443 \mathrm{S10})$ is at $12^{\circ}$ $S$ and shows many small and well-preserved craters. The right image (575B57) is at $49^{\circ} \mathrm{S}$ and shows no small craters and a large crater with a very subdued rim. These are the same images used by Carr (1996) to demonstrate terrain softening. 


\subsection{Conclusion}

We have developed a 1-D thermal model for the near-surface ground of Mars to estimate the extent of stable ground ice. Thermal properties of the ground are allowed to vary with depth. We have treated the atmosphere with enough detail to accurately reproduce surface temperatures found with models that treat the atmosphere more thoroughly, for example the models used to determine thermal inertia (Haberle and Jakosky 1991), yet our model runs in a fraction of the time. A convergence algorithm rapidly finds a steady-state solution.

We have developed a scheme that allows the near-surface atmosphere to have a different water content relative to the whole atmospheric column. Cold nighttime temperatures at the surface can deplete the water vapor in the lowest part of the atmosphere by forming frost on the surface. This depletion reduces the average water vapor density in the part of the atmosphere that is in diffusive contact with ground ice, relative to the equivalent density of the atmospheric column.

We use a new way to estimate the amount of water in the martian atmosphere for any epoch. We found that the present, typical abundance of water vapor in the martian atmosphere $(\sim 14 \mathrm{pr}-\mu \mathrm{m})$ is equal to the water vapor carrying capacity of the atmosphere over surface ice at the north pole. This assumes ground with surface ice has high albedo and high thermal inertia. We propose that the average water content of the martian atmosphere at any epoch can be estimated by the minimum in the water carrying capacity 
of the atmosphere anywhere over the surface of Mars. In epochs of low obliquity, the minimum water carrying capacity is at one pole or the other, while at high obliquities the minimum is at low latitudes. Atmospheric water contents at high obliquities from this approach agree well with results from GCMs that are considerably more sophisticated than our approach.

Using our models, we calculate the distribution of ground ice on Mars for many different scenarios. We also evaluate the significance of various aspects of the model. The inclusion of the vapor depletion scheme was found to reduce the extent of stable ground ice by several degrees of latitude in the present epoch. Modifying the water content of the atmosphere, either by correcting for elevation or using the observed water content, only made minor changes to the ice extent $\left(\sim 1^{\circ}\right.$ of latitude). The effect of allowing atmospheric thickness to vary with elevation, which modifies the attenuation of sunlight and thermal radiation on the surface, was negligible (a change of $\sim 0.1^{\circ}$ of latitude).

The presence of a high-thermal inertia ground ice at shallow depths, as determined by stability to vapor diffusion, was found to effectively negate changes in thermal model results due to changes in the thermal inertia of the top ice-free layer at the surface. For example, the average surface temperature, the maximum seasonal $\mathrm{CO}_{2}$ build up and the Crocus date for a particular location were found to be almost independent of the thermal inertia of the ground when the ground ice was present.

We tested the sensitivity of the calculated stable ice distribution to the construction of the vapor depletion scheme that we use. Variations in the amount of 
water vapor that can be held in the atmospheric column relative to surface temperatures made negligible changes to the effective water vapor ratio in the near-surface atmosphere and depth at which ground ice is stable. Changes in the extent of stable ground ice will be much less than the change when the vapor depletion scheme is applied or not applied. If there were processes that can systematically enhance or deplete the average water content of the near-surface atmosphere relative to the rest of the atmospheric column, the changes in the stable ice extent would still be small. The change in ice extent when the amount of water in the atmosphere was doubled (or halved) was only of the order of a few degrees in latitude. From this we conclude that while the construction of our vapor depletion scheme may miss details in its simplicity, the effects of these details on the stable ground ice extent are insignificant.

When testing the extent of ground ice with high atmospheric water contents, with the orbit and obliquity from the present epoch, our model does not allow ice to extend to low latitudes. The vapor depletion algorithm does not allow the atmosphere to ever become super saturated; this restriction effectively places a limit on the amount of water that can be put into the atmosphere. This saturation limit is reached at some locations with out allowing ground ice to become stable, particularly in low- to mid-northern latitudes.

We estimate the variations in the ground-ice distribution as Mars precesses within its current orbit and current obliquity. As the position of perihelion changes, there are slight changes in surface temperature and in the atmospheric water content. The resulting ice distribution is a combination of these two effects. The atmospheric water content of 
Mars at present is near the minimum within the precession cycle. The limit of ground ice in the northern hemisphere varies $\sim 14^{\circ}$ in latitude over a precession cycle while the limit in the south only varies $\sim 6^{\circ}$. The significance of this cycle is that while the obliquity is expected to have a more significant effect in the long term, over the past few hundred thousand years obliquity has been relatively constant. The position of perihelion can induce changes in the extent of ground ice that are of the same order as the changes in ice extent due to the small obliquity changes in this time.

When estimating the atmospheric water content as a function of obliquity, we found that our content was lower than previous estimates by Mellon and Jakosky (1995). At low obliquities, the two estimates are in general agreement, but at high obliquities our approach gives a water content that is lower by over an order of magnitude. At higher obliquities we find that the atmospheric water content is controlled by the atmospheric water carrying capacity over surface ice at low latitudes. Our results using low-latitude surface ice are consistent with results obtained with general circulation models (Mischna and Richardson 2005). As a consequence of these lower atmospheric water contents and the inclusion of water vapor depletion of the near-surface atmosphere, we find ground ice is not as extensive at high obliquities relative to previous results. Mellon and Jakosky (1995) found ground ice became stable globally at obliquities as low as $32^{\circ}$, which does not occur in our model. At the highest obliquity tested in our model $\left(45^{\circ}\right)$, ground ice only extended to latitudes of 15 to $20^{\circ}$ in the north and $25^{\circ}$ in the south over most of the globe. Ground ice does not become globally stable for any of the models tested with vapor depletion or our lower water contents. 
The absence of stable ground ice near the equator and low latitudes in epochs of high obliquity is not inconsistent with the surface ice deposits shown by GCMs in these epochs (e.g. Mischna et al. 2003). In fact, our approach using the minimum in the atmospheric water carrying capacity also implies ice is stable on the surface at low latitudes in high obliquity epochs. The processes that determine where ground ice is stable are very different to the processes that form deposits of ice on top of the surface. Ice is deposited onto the surface, presumably, by precipitation from the atmosphere and is driven by atmospheric circulation while ground ice is stable just when there is a net zero flux in vapor diffusion to the atmosphere. The precipitation of water from the atmosphere is not included in our models to calculate where ground ice is stable, so it is entirely possible that surface ice deposits may form where we indicate ground ice would be unstable.

Finding that ground ice does not become globally extensive is consistent with the extent of ground-ice related landforms, such as dissected mantle and terrain softening that are not found at low latitudes. Recent dynamic models indicate that Mars has spent perhaps most of its history at high obliquities (Laskar et al. 2004). If ground ice had been stable globally at high obliquities, then it would be expected that evidence of ice-related processes such as terrain softening would also be global. Terrain softening is not global and our model explains why ground ice was absent at low latitudes over the history of Mars. The extents of stable ground ice in past and present epochs coincide with the extents of various dissected mantle terrains identified and mapped by Milliken and Mustard (2003). The poleward limit of the "knobby" and "wavy" terrains of mantle is 
approximately the same as the limit of stable ice at present. At these locations, ground ice is unstable at any depth in the present epoch. The presence of lag material over ice may slow the rate of sublimation but degradation will still continue if there is still diffusive contact between the ice in the subsurface and the atmosphere. The extent of the "scalloped" mantle terrain lies roughly between the extent of stable ground ice at present and the extent at an epoch with the lowest recent obliquity $\left(\sim 15^{\circ}\right)$. At these locations, ground ice should be stable in the present epoch and a thin lag cover is all that would be required to shut off any net sublimation loss. Presumably, these pits have formed during brief periods of low obliquity in the recent geological history.

The models presented here to predict where ground ice is stable are all consistent with the idea that Mars is predominantly cold and dry. The amount of water in the atmosphere is determined by surface ice at the coldest place on the planet where the relative humidity is highest - this happens to be the north pole in the present epoch. The water reservoirs in close contact with the atmosphere respond to this water content, such the depth and the extent of ground ice. 


\section{CHAPTER 3}

\section{NEAR-INFRARED SPECTROSCOPY OF HIMALIA}

\subsection{Introduction}

Spectra of the irregular jovian satellite Himalia (JVI) were obtained with the Visual and Infrared Mapping Spectrometer (Miller et al. 1996; Reininger et al. 1994) onboard the Cassini spacecraft on December 18-19, 2000. These observations were part of the Cassini Jupiter Flyby during which the jovian system was surveyed while the spacecraft was en route to Saturn.

We present here the first spectrum beyond $2.5 \mu \mathrm{m}$ of Himalia or of any other irregular satellite of a planet in the outer Solar System. Jupiter's irregular satellites fall into two main families related by semi-major axes, inclinations and orbital directions. Himalia is a member of the inner, prograde family and orbits Jupiter at an average distance of $1.14 \times 10^{7} \mathrm{~km}$, or 161 jovian radii.

Himalia was discovered in 1904 by Perrine. Almost 3/4 of a century later observations at $20 \mu \mathrm{m}$ by Cruikshank (1977) determined the size of Himalia to be $85 \mathrm{~km}$, making it the largest irregular satellite of Jupiter. With this size, the visual brightness of Himalia implies a geometric albedo of 0.03 in the $\mathrm{V}$ band. The rotational period of Himalia is between 9.2 and 9.8 hours (Degewij et al. 1980). Based on a multicolor 
photometric survey, Tholen and Zellner (1984) found the spectrum of Himalia was more similar to that of C-type asteroids than P- or D-types or other asteroids from the outer belt. This result was borne out by near-infrared, K-band spectroscopy by Dumas et al. (1998), Brown (2000) and Geballe et al. (2002), who show a dark, flat spectrum of Himalia like that of C-type asteroids. A weak absorption at $0.7 \mu \mathrm{m}$ in Himalia's spectrum has been attributed to oxidized iron by Jarvis et al. (2000).

Multicolor photometry and spectroscopy of the irregular jovian satellites as a whole have had mixed results. Initial broadband photometry (Tholen and Zellner 1984) suggested that all the irregular jovian satellites were C-type asteroids. However, Smith et al. (1981) started to suggest that some members of the retrograde family were too red to be C types. Spectroscopy by Luu (1991) implied that there was a similarity between the irregular satellites of Jupiter and Jupiter's Trojan asteroids. Luu identified C- and D-type spectra in both prograde and retrograde families. Using broadband photometry again, out to the near-infrared, Sykes et al. (2000) and Rettig et al. (2001) now suggest prograde satellites all show C-type colors while the retrograde family shows more variation, including $\mathrm{C}$ and $\mathrm{D}$ types. What are missing are albedo calculations. More measurements of thermal fluxes and geometric albedos would be useful to see if all the satellites that have flat, C-like colors also have low, C-like albedos.

The origin of the Jupiter's irregular satellites is still uncertain, the most popular hypothesis is that the satellites were captured via gas drag just before the jovian protoatmosphere collapsed (Pollack et al. 1979a). The question of where the parent body(s) of the Jupiter's irregular satellites originated is still open to conjecture. Was the parent body 
from the Main Asteroid Belt, the outer belt, the Trojan clouds, even the Kuiper Belt, or was it a left over planetesimal from near-Jupiter space? Different materials are expected in these different regions of the Solar System so knowledge of Himalia's surface composition could constrain the origin of the parent body of Himalia and other jovian irregular satellites.

\subsection{Observations}

The VIMS instrument onboard Cassini (Miller et al. 1996; Reininger et al. 1994; Miller et al. 1996) took spectra of Himalia while it was near its closest approach to Himalia during the Jupiter flyby. A summary of the observation parameters is provided in Table 1.

\begin{tabular}{|l|l|}
\hline Parameter & Value \\
\hline Instrument & VIMS - Cassini spacecraft \\
\hline Cube Size & $12 \times 12$ spatial pixels x 256 \\
& channels \\
\hline Pixel Size & 0.5 milliradians $(\sim 10 \mathrm{x}$ size of \\
& object) \\
\hline Wavelength Range & 0.8 to $5.12 \mu \mathrm{m}$ \\
\hline Channel Bandpass & $\sim 0.016 \mu \mathrm{m}$ \\
\hline Time Observed & 18 Dec $200020: 30$ UTC to \\
& 19 Dec 2000 01:10 UTC \\
\hline Phase Angle & 70 degrees \\
\hline Distance to Object & 4440 000 km \\
\hline Heliocentric Distance of Object & 5.04 AU \\
\hline Integration Time per Cube & 0.640 seconds \\
\hline
\end{tabular}

Table 6 Observation parameters for Himalia 
Observations occurred over a period of 4 hours, and 41 image cubes were collected. Because identification of Himalia in the image cubes was difficult due to low signal and drift in the spacecraft pointing, Himalia was identified in only 14 of the image cubes. Problems in pointing arose because the Cassini spacecraft went into fault protection mode, and consequently switched from reaction wheels to thrusters for attitude control. Because attitude control is much coarser on thrusters, Himalia was out of the field of view of VIMS in many of the 41 cubes.

An additional source of uncertainty in spectral measurements using VIMS was due to cosmic ray hits on its detector. These hits produced an avalanche of charge carriers in VIMS' InSb array detector elements resulting in spurious signals that were orders of magnitude greater than the signal from the object. Fortunately, a cosmic ray hit typically only affected a small number of spectral channels in a single pixel, thus it was possible to remove affected channels and still analyze the data from the unaffected channels.

The "sky" background for each Himalia spectrum collected was determined by averaging the signal in all the pixels in the field of view except for the Himalia pixel for each of the 256 channels. The standard deviation of the background values was used to identify outliers to be removed from the background average. This took out any cosmic ray hit signals from the background.

No other objects were ever in the field of view. All of the light from Himalia was assumed to be in one pixel. The angular size of Himalia was larger than the diffraction limit for all VIMS wavelengths, and this size was about one tenth that of a single VIMS 
pixel. It was certainly possible that some light from Himalia was lost to adjacent pixels in some frames. The problem was that in a single frame the signal from Himalia was comparable to the magnitude of the noise from a single pixel. Adding the adjacent pixels potentially gains a small increase in signal but also increases the noise by a factor of 3 .

Cosmic ray hits were removed from the Himalia pixel with a moving boxcar. A boxcar of width 15 was allowed to run along the 256, background-subtracted channels. The difference between the signal in the center channel and the average of the boxcar was compared to the standard deviation $(\sigma)$ of the signals within the boxcar. If the difference was greater than $2.325 \sigma(<2.5 \%$ of a normal population $)$, the center channel was nulled. This process removed cosmic ray hits from the Himalia signal. By subtracting the background before applying this spike filter we avoided losing channels that had spikes due to the background that was subtracted from the field of view before the data were delivered from the instrument.

Once each frame had been background subtracted and despiked for cosmic ray hits, the individual spectra of Himalia from all the frames were averaged together. Channels that had been nulled were excluded from this average.

Object intensity was calculated using pre-launch and in-flight instrument response calibrations. Details of the instrument calibration are provided in McCord et al. (2004).

The uncertainty of the signal for each channel is taken as the standard deviation of the values from all of the corrected image cubes used. To improve the signal-to-noise ratio (SNR), the spectrum was convolved with a Gaussian whose full width at half maximum (FWHM) was four times the original VIMS channel spacing, $0.064 \mu \mathrm{m}$ and 
$\sim 0.016 \mu \mathrm{m}$ respectively. This improved the SNR at the expense of spectral resolution. The resolution of the raw VIMS data is about a channel width, so the spectral resolution of the convolved spectrum is practically the FWHM of the Gaussian.

A correction was also made for the thermal radiation from Himalia using a model based on Himalia's size, rotation period, bolometric bond albedo, heliocentric distance, and a thermal inertia plausible for a minor planetary body (see Appendix A). Because Himalia's obliquity is unknown, we calculated models representing two end members: 0and 90 -degree obliquity. The 0 -degree obliquity case represents a rough lower limit to the thermal flux in our raw spectrum, while the 90-degree obliquity case represents a rough upper limit. The reflectance spectrum shown in Figure 44 has a thermal correction that is the average of the 2 limiting cases. The magnitude of the thermal correction to the spectrum is greatest for longest wavelengths. In the last VIMS channel at $5.1 \mu \mathrm{m}, 30 \%$ of the observed signal was attributed to thermal radiation when the spectrum was corrected for Figure 44. The thermal correction tapered down to just $2 \%$ of the observed signal at $4.3 \mu \mathrm{m}$. While the thermal correction is within the error bars at all wavelengths, it does account for a significant part of the averaged signal in the longest wavelength channels. 




Figure 44 Normalized reflectance spectrum of Himalia as recorded by VIMS. Individual VIMS channel averages are shown with crosses. To improve the SNR these averages were convolved with a Gaussian. Convolved data are shown with diamonds and one standard deviation error bars were calculated from the standard deviations of each VIMS channel average. This spectrum has been corrected for thermal radiation which adjusts the longest wavelength channels. 


\subsection{Results}

Figure 44 shows the normalized reflectance spectrum of Himalia taken by VIMS. This spectrum was first published in Brown et al. (2003). Shown are both the average reflectance calculated for each VIMS channel and the convolution of the channels to improve the SNR. The error bars show one standard deviation in the convolved spectrum based on the distribution of the values in each channel.

Reflectance is often given as geometric albedo, which is a useful property of a surface. Geometric albedo is the ratio of the reflected intensity of a body observed at 0degree phase relative to a Lambert disk of the same projected cross section, however the VIMS Himalia observations were at phase of 70 degrees. Correcting for this phase angle is the greatest source of uncertainly in estimating a geometric albedo for Himalia with VIMS data. The reflectance observed at 70 degrees could be converted to reflectance at zero degrees if the phase function of Himalia was known. When an object's phase function is poorly known, as is the case for Himalia, the brightness in magnitudes can be approximated as a linear function with phase angle. C-type asteroids and other small dark Solar System objects have values of the around 0.035 to 0.040 magnitudes-perdegree (Helfenstein and Veverka 1989). Using these values and a body radius of $85 \mathrm{~km}$ (Cruikshank 1977) we determine the geometric albedo to be around 0.07 to 0.10 in the K band. These magnitudes-per-degree values are based on observations in visual wavelengths and may not be correct in the infrared, as inferred in the following 
discussion of phase reddening. Phase reddening would mean our K-band geometric albedo is overestimated.

\subsection{Discussion}

There are some differences between the spectrum presented here and previous spectra from the K band observed from the ground (Dumas et al. 1998; Brown 2000; Geballe et al. 2002). The results from the ground have higher resolution and better SNR; the VIMS data cover a wider wavelength range. The VIMS spectrum is redder than most spectra from the ground. The K-band data from Brown (2000) show an increase in the reflectance of $\sim 10 \%$ from 1.4 to $2.4 \mu \mathrm{m}$. The Dumas et al. (1998) spectrum is flatter with a slope $<2 \%$. For the same wavelength band VIMS data show an increase in reflectance of $\sim 18 \%$ when observed at a phase angle of 70 degrees. The increased slope in VIMS data could be attributed to phase reddening. The spectrum from Geballe et al. (2002) from 1.95 to 2.5 shows a red slope more like VIMS than the other spectra from the ground. The differences in red slope observed from the ground could be due to a number of reasons, including irregularities of the target body.

Phase reddening of asteroid colors has been documented (e.g., Gehrels 1967; Taylor et al. 1971; Zappala et al. 1979; Tedesco and Sather 1981), but these studies did not extend into the infrared. Clark et al. (2002) observed a phase reddening of the surface of the asteroid Eros over phase angles from 0 to 100 degrees with the NEAR infrared spectrometer. Over this phase range there was a noticeable increase in redness with 
increasing phase angle over the wavelengths 0.8 to $2.4 \mu \mathrm{m}$. This suggests that the reddening we see in Himalia could be due to the phase effects and would not necessarily be seen in a geometric albedo if it were observed at a 0-degree phase angle.

The strongest suggestion of an absorption feature in the VIMS Himalia spectrum is at $3 \mu \mathrm{m}$. The depth of the absorption is the same size as the error bars, though the feature is spread over several channels suggesting that the feature is real. A fundamental vibration of water corresponds to $3 \mu \mathrm{m}$ so the VIMS spectrum suggests the presence of water on the satellite surface. Due to the low SNR and possible artifacts in the instrument calibration, this identification is not secure. One might also be tempted, based on these data, to try to distinguish between free water ice and water of hydration, but we believe that isn't prudent. Likewise we refrain from commenting on the data beyond 4 $\mu \mathrm{m}$ because of the low SNR there. 




Figure 45 The convolved reflectance spectrum (diamonds) is overlain with model spectra produced with Hapke theory and optical constants of 2 candidate materials. The solid line uses optical constants for water ice; the dashed line uses optical constants for the hydrated-mineral serpentine. In both models, ice and minerals were mixed with a featureless red material to improve the fit to the continuum. 
Overlain on the convolved VIMS spectrum in Figure 45 are two spectral models, one has water ice and the other a hydrated mineral, serpentine $\left(\mathrm{Mg}_{3} \mathrm{Si}_{2} \mathrm{O}_{5}(\mathrm{OH})_{4}\right)$. Both models are a mixture with a second component, a featureless red material, to improve the fit to the observed spectrum. Models were produced with optical constants and Hapke theory (1993). Water ice optical constants came from Warren (1984); these ice constants are for temperatures warmer than the surface of Himalia, however they cover the full wavelength range of this spectrum. Serpentine optical constants came from Clark et al. (1993) and Mooney and Knacke (1985). The red material was completely artificial, though it had the approximate optical properties of carbonaceous material. The first model has $\sim 4.5$ weight $\%$ water ice mixed with red material in $10-\mu \mathrm{m}$ grains. The small grains suppress the 1.5 - and $2.0-\mu \mathrm{m}$ features that are often used to identify water ice in the outer Solar System. The second model has $\sim 10$ weight $\%$ serpentine mixed with red material, also with $10-\mu \mathrm{m}$ grains.

Both the ice and the hydrated-mineral model have absorption features around 3 $\mu \mathrm{m}$ to give general matches to the VIMS spectrum. Neither of the models gives a convincing unique fit, as to be expected in data with a low SNR. The shape of the absorptions of water ice and hydrated minerals are different, so in principal the two could be distinguished in reflected spectra. The serpentine model matches the VIMS Himalia spectrum well from 2.5 to $3.1 \mu \mathrm{m}$. At $3.3 \mu \mathrm{m}$ there is a suggestion of an absorption that doesn't match the serpentine model. If the 3.3- $\mu \mathrm{m}$ data were part of the same $3-\mu \mathrm{m}$ feature, it could all be modeled with water ice, but this would mean data at $3.1 \mu \mathrm{m}$ is 
erroneous. It could be that it is the data at $3.3 \mu \mathrm{m}$ that is awry but we can not know this without more data. This precludes us from distinguishing between water ice and hydrated minerals on the surface of Himalia.

Previous K-band spectroscopy would have detected ice combination bands at 1.5 and $2.0 \mu \mathrm{m}$ if ice were abundant on the Himalia's surface. No such detection has been made. Brown (2000) matched his data with a model containing $0.1 \%$ ice, which was consistent with an ice-free surface. Calculations by Geballe et al. (2002) that allow for the suppression of combination bands by small grain size, demonstrate that the surface could have up to $10 \%$ water ice and not show any water features in the K-band.

\subsection{Conclusion}

While our data do not confidently identify water on Himalia, the absorption around $3 \mu \mathrm{m}$ is suggestive that water may be present on the surface. The presence or absence of water, and the form of the water on Himalia's surface, could help constrain the nature of the origin of Himalia and the rest of the irregular satellites.

The presence of any water on the surface of Himalia would support the argument that the jovian irregular satellites are unrelated to the outer belt asteroids, namely the Pand D-type asteroids such as the Trojans. From multicolor surveys, P and D types are redder than most jovian irregulars. L-band spectroscopy of D-type asteroids (Cruikshank et al. 2001; Emery and Brown 2003) has not yet detected any water in any form. Vilas (1994) argues that Himalia should have a water of hydration feature based on 
a shallow feature at $0.7 \mu \mathrm{m}$ in Himalia's spectrum and a correlation found in the Main Belt.

It is thought (as discussed in Cruikshank et al. 2001) that the outer belt asteroids originally formed with substantial ice. This ice was lost, at least from the observable surface, by a combination of processes such as: solar heating, impact gardening, and solar wind sputtering. For Himalia to show any spectral signature of water, the surface material could contain water trapped in minerals such as water of hydration.

Alternatively, there could be a subsurface source of ice that replenishes ice lost via the processes mentioned before.

The prevalence of water of hydration observed in C-type asteroids decreases with heliocentric distance. This effect is believed to be due to a decrease in solar heating. If Himalia has water of hydration then it presumably was originally a Main Belt asteroid before being perturbed and captured into the jovian system. If Himalia has water ice, this would indicate that it was originally ice-rich, more so than outer belt asteroids, and that it formed further out in the Solar System.

Differentiating between water of hydration and water ice can be done spectroscopically. While the VIMS spectrum of Himalia does not have the signal-tonoise to resolve this question, it is the closest spectroscopic data to doing so. No life was found on Himalia. 


\section{CHAPTER 4}

\section{FUTURE WORK}

It was with mixed emotions that I realized several months ago, just how little of the work that I have done over the past years will actually be presented in detail in my dissertation. On one hand, there was disappointment that I don't get to show off everything that I have worked on. But this was soon replaced by excitement at the idea that there is so much still to do.

Here I use the "Future Work" chapter as an excuse opportunity to describe some ideas and results from projects that were started and where interesting progress was made, though the project did not quite make it to the level where it could be presented as a complete chapter here in a planetary science dissertation. Some of these projects are a natural follow-on from the work I have presented here. Other projects use the thermal models I have developed as a start but require significant development and extensions. It is my sincere hope that I will be able to continue working on, even just a fraction of what I describe in this Chapter as I start post-docing and doing research in new topics. 


\subsection{Polar Thermal Models}

Usually in planetary science data is scarce and there very little point in creating more than a simple model to explain the observations. However, I have found an application for near-surface thermal models where it is clear that the data is trying to say something that is beyond the ability of simple thermal models to understand or reproduce.

Over the martian polar regions there is good coverage of surface temperature data due to the polar orbits of present martian spacecraft. Simple thermal models no longer explain data available in these regions. New processes need to be included in the models to explain the results and these processes can be related to various properties of the surface.

When I initially started playing with these ideas, I was trying to explain anomalies that appear in the thermal inertia of high-latitude regions (Putzig et al. 2005). Estimating the thermal inertia in the polar regions is difficult because seasonal frost covers the region for most of the year. Also, most models used to determine thermal inertia use the diurnal thermal cycle which is not significant as you approach the pole.

What I found, and demonstrated in an LPSC abstract (Chamberlain and Boynton 2005), is that two-layer thermal models do a much better job at fitting TES temperatures (that were provided by Tim Titus) over the summer relative to one-layer models. I was able to show that the thermal inertia value inferred from surface temperatures, if using a one-layer model as a reference, would vary according the time of season, which did not occur when using a two-layer model. As an added bonus to finding a better thermal 
inertia, my method also found the depth to ground ice. This now nicely compliments my other work estimating the depth to stable ice. Preliminary results indicate that ice depth determined from surface temperatures is greater than ice depths based on just the thermal model and atmospheric water content. Perhaps one of these answers will be close to the ice depth that is found when Phoenix lands in 2008.

Surface temperature observations in the spring over the poles are hard to interpret. The timing of the warming of the surface due to loss of seasonal frost predates the disappearance of frost observed by orbiting cameras by several degrees of $L_{s}$. There is an increase in surface temperatures before the seasonal frost disappears that can not be explained by atmospheric effects. Something is going on at the surface during this season that significantly increases the thermal radiation from the surface while seasonal frost (buffered at $\sim 149 \mathrm{~K}$ ) is still present. Perhaps there are small patches of frost-free high thermal inertia or a layer of water frost forms over the seasonal $\mathrm{CO}_{2}$ that is able to raise the surface temperature as observed by TES. This is a problem that thermal models can address.

Also during the spring, $\mathrm{CO}_{2}$ seasonal frost disappears and the surface temperature increases rapidly. In models this increase is almost instantaneous; not surprisingly, in the actual data the increase is slower. Understanding just why this is the case is also something thermal models can address. As mentioned in the ground-ice chapter, when ground ice is present, variations in thermal inertia do not change the timing of the disappearance of seasonal frost significantly. The reason for the slow rise in temperature will probably be related to the range of slopes within the instrument footprint. 


\subsection{Vapor Diffusion}

Among the other projects that almost came to be was a model of vapor diffusion through the martian regolith. I spent quite some time developing a model that coupled the diffusion of water vapor with a thermal model so that there was a feedback between the distribution of ice and the temperatures.

Thermal properties of the ground were modified as ice condensed within the ground, and new temperature profiles were generated. The model was designed to be able to run for thousands of model years in order to simulate the formation or desiccation of the ground ice. What was found was that the model becomes unstable just when the ground ice approaches a depth at which it is steady state with the water content of the atmosphere. I developed a smoothing algorithm to avoid the unrealistic oscillations in the depth to ice that would develop. Preliminary results were shown in another LPSC abstract (Chamberlain and Boynton 2004).

A robust thermal model that includes vapor diffusion has a number of useful applications. For example,

- What is the expected exchange of water from buried ground ice with the atmosphere on a seasonal timescale? This has implications for GCM models that attempt to replicate Mars water cycle.

- How long does it take for significant deposits of water ice to from in the martian ground where vapor diffusion is the dominant process? Or on the other hand, 
- How long would ground ice deposited at low latitudes survive while ground ice is unstable? This is a particularly hot topic that is begging for a short paper to be written about. Low-latitude water-equivalent contents at derived from the GRS neutron spectrometer are higher than the same contents based on hydrogen gamma rays (Feldman et al. 2004b; Boynton et al. 2002). This has lead some to speculate that a recent epoch of high water content in the martian atmosphere may have formed deposits of low-latitude ground ice (Jakosky et al. 2005). While it seems unlikely that ground ice would be able to persist within $10 \mathrm{~s}$ of $\mathrm{cm}$ of the surface for 1000s of years, a short paper presenting a model to support this argument would be useful to the Mars research community right now.

\subsection{Comparison to GRS Results}

I'm sure that when Bill Boynton first suggested that I look at theoretical models of ground-ice distribution when I started working for him 3 years ago his intention was that I would be using these models to make sense of the water distribution observed with his instrument, the Gamma Ray Spectrometer. So it is somewhat of a shame that I still have not done this! This is in no small part due to the fact that the theoretical distribution of ground ice derived over ten years ago (Mellon and Jakosky 1993) appeared to already be in good agreement to the GRS results.

I would argue that this agreement is qualitative more than quantitative. While the ground ice derived by theoretical models seem to model the extent of ground ice over the 
martian globe very well, it is not clear that it matches the depth to ice over the globe. In the GRS data that has been formally published there has been little depth information. However, Bill Boynton is in the process of developing a new set of results that will be able to map the depth to ground ice and the water content of the ice-rich layer over the martian globe. Preliminary results have been presented at conferences (Boynton et al. 2005). Once all the corrections are made, the GRS depth to ice can then be compared to the theoretical depths to stable ice. If ice is found closer to the surface than the depth to stable ice, this could be a location where ice is subliming and a source in the water cycle. Likewise, ground ice deeper than where ice is stable can act as a sink, though rates of vapor exchange may be so slow the source/sink effects will be negligible.

\subsection{Miscellaneous}

The other project that I need to complete here in the US is the running of a century; that is a 100-mile ultra marathon. This will compliment the other events I have completed here: marathons, ironman triathlon, 250-mile bike ride, (and fatherhood!?). It is the same dumb determination that I use in these events that helped me complete this degree. 


\section{APPENDIX A: THERMAL MODEL APPLIED TO CORRECT THE HIMALIA SPECTRUM}

The calculation of the contribution of emitted thermal radiation to the observed spectrum of Himalia was made using a program model called "Thermod" created by one of us (RHB) to allow calculation of thermal flux from a variety of solar system bodies under a variety of conditions. The basic engine of the program is a Crank-Nicholson finite differences solution of the thermal diffusion equation for the case of variable thermal conductivity. The basic form of that equation used in "Thermod" is:

$$
\rho C \frac{\partial T}{\partial t}=\frac{\partial}{\partial z}\left(\kappa(z) \frac{\partial T}{\partial z}\right)+E(z),
$$

where $\rho$ is density, $\mathrm{C}$ is heat capacity, $\mathrm{T}$ is temperature, $\mathrm{t}$ is time, $\mathrm{z}$ is depth in the modeled surface, $\kappa$ is the thermal conductivity, and $\mathrm{E}$ is an energy source term that can represent other kinds of energy sources such as radioactive decay, or a solid-state greenhouse effect.

The upper boundary condition in "Thermod" is user selectable, but presently includes both a radiative/conductive upper boundary, and a radiative/conductive/sublimative upper boundary, both of which demand energy conservation. The former boundary condition was used here and it is given by: 


$$
S_{0}(1-A) \cos (\zeta)\left(\frac{R_{0}}{R}\right)^{2}=\sigma \varepsilon T^{4}+\left.\kappa \frac{\partial T}{\partial z}\right|_{z=0}
$$

where $S_{0}$ is the solar constant at distance $R_{0}, A$ is the bolometric bond albedo, $\cos (\zeta)$ is the cosine of the solar zenith angle relative to the normal to a unit-area surface element, $\sigma$ is the Stephan Boltzmann constant, and $\varepsilon$ is emissivity.

The lower boundary in the model is assumed to be well below any periodic temperature fluctuations on the timescales of interest (either diurnal or seasonal) and is given by:

$$
\left.\frac{\partial T}{\partial z}\right|_{z=\infty}=0
$$

The finite differences calculation is done on a linear depth grid of arbitrary extent and resolution (but not less than 5 steps per diurnal or seasonal skin depth), and on a surface grid that allows a minimum of 1 degree steps in latitude and 0.125 degree steps in longitude (i.e., rotational phase). Thermod allows the user to include all of the parameters relevant to bodies in heliocentric orbit for the purposes of obtaining a solution for the temperature distribution in longitude, latitude, and depth as a function of time (i.e., over an orbit). A typical calculation is run for the number of rotations and orbital periods required so that the temperature distribution with depth converges to a full periodic solution.

For the case of Himalia considered here we use the following thermal parameters: $\kappa=160 \mathrm{erg} \mathrm{s}^{-1} \mathrm{~cm}^{-1} \mathrm{~K}^{-1}, \rho=1.0 \mathrm{gm} \mathrm{cm}^{-3}$ (silicate particulates at about $60 \%$ porosity), and $\mathrm{C}=8 \times 10^{6} \mathrm{ergs} \mathrm{gm}^{-1} \mathrm{~K}^{-1}$. Such a choice of parameters gives a thermal inertia $(\kappa \rho C)^{1 / 2}$ 
of about $3.6 \times 10^{4} \mathrm{erg} \mathrm{cm}^{-2} \mathrm{~K}^{-1} \mathrm{~s}^{-1 / 2}$, which is similar to that of the Moon. We estimated Himalia's bolometric bond albedo from the relationship:

$$
A=p q
$$

where $\mathrm{p}$ is the object's visual geometric albedo (0.03), and $\mathrm{q}$ is the phase integral (assumed to be $\sim 0.6$ in analogy with asteroids). The semi-major axis and eccentricity was assumed to be that of Jupiter, the rotation period 9.5 hours, and an effective obliquity of 0 and 90 degrees. The heliocentric distance of Himalia when the observations were made was 5.04 AU. We call it effective obliquity because we ignore Himalia's motion around Jupiter for this calculation. The extent of the depth grid was 20 seasonal skin depths divided into 5 equal steps per skin depth. Finally, although Himalia is clearly irregular in shape, we used a spherical geometry for the model whose diameter is equal to the $85 \mathrm{~km}$ effective cross section determined by Cruikshank (1977). The model was run for the equivalent of 1 Jupiter year to ensure convergence of the temperature distribution at depth. The results are shown in Figure 46. 


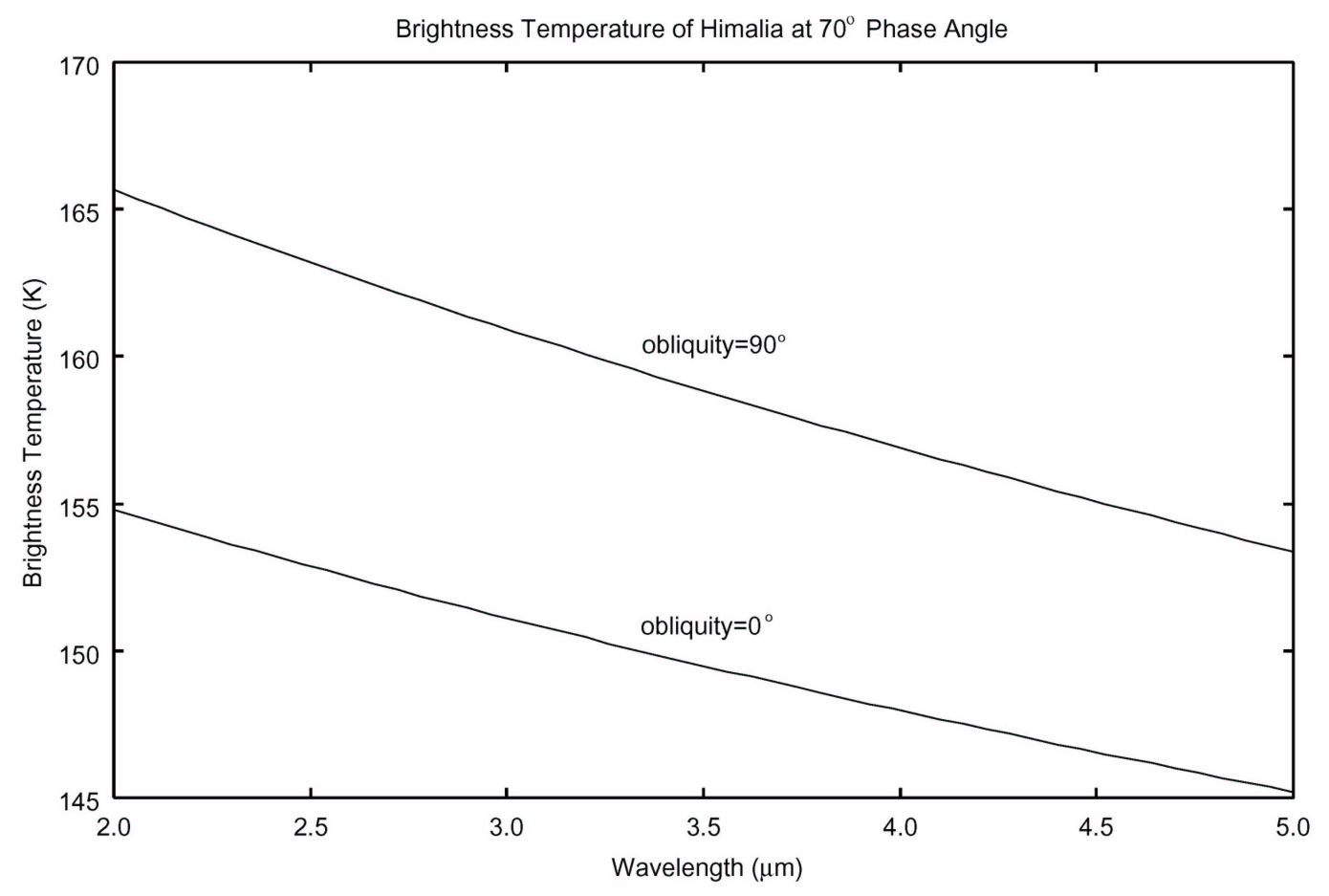

Figure 46 Thermal radiation calculated from "Thermod" for 2 limiting cases of obliquity. 


\section{REFERENCES}

Armstrong, J. C., Leovy, C. B., Quinn, T., 2004. A 1 Gyr climate model for Mars: new orbital statistics and the importance of seasonally resolved polar processes. Icarus $171,255-271$.

Barker, E. S., Schorn, R. A., Woszczyk, A., Tull, R. G., Little, S. J., 1970. Mars: Detection of atmospheric water vapor during the southern hemisphere spring and summer season. Science 170, 1308-1310.

Black, R. F., 1976. Features Indicative of Permafrost. Annual Review of Earth and Planetary Sciences 4, 75.

Boynton, W. V., Feldman, W. C., Squyres, S. W., Prettyman, T. H., Brnckner, J., Evans, L. G., Reedy, R. C., Starr, R., Arnold, J. R., Drake, D. M., Englert, P. A. J., Metzger, A. E., Mitrofanov, I., Trombka, J. I., d'Uston, C., Wänke, H., Gasnault, O., Hamara, D. K., Janes, D. M., Marcialis, R. L., Maurice, S., Mikheeva, I., Taylor, G. J., Tokar, R., Shinohara, C., 2002. Distribution of Hydrogen in the Near Surface of Mars: Evidence for Subsurface Ice Deposits. Science 297, 81-85.

Boynton, W. V., Kim, K. J., Drake, D., Reedy, R. C., Janes, D., Kerry, K., Williams, R., Crombie, K., The Grs Science Team, 2005. Determination of Both Depth and Ice Content of Sub-Surface Ice in the Polar Regions. 36th Annual Lunar and Planetary Science Conference 36, 2154.

Boynton, W. V., Taylor, G. J., Evans, L. G., Reedy, R. C., Starr, R., Janes, D. M., Kerry, K., Drake, D. M., Kim, K. J., Williams, R. M. S., Crombie, K., Dohm, J. M., Baker, V., Metzger, A. E., Karunatillake, S., Keller, J., Arnold, J. R., Brückner, J., Englert, P. A. J., Gasnault, O., Sprague, A. L., Squyres, S. W., Trombka, J. I., d'Uston, L., Wänke, H. 2006. Concentration of H, Si, Cl, K, Fe, and Th in the low and mid latitude regions of Mars. J. Geophys. Res. in preparation.

Brown, M. E., 2000. Near-Infrared Spectroscopy of Centaurs and Irregular Satellites. Astron. J. 119, 977-983.

Brown, R. H., Baines, K. H., Bellucci, G., Bibring, J.-P., Buratti, B. J., Capaccioni, F., Cerroni, P., Clark, R. N., Coradini, A., Cruikshank, D. P., Drossart, P., Formisano, V., Jaumann, R., Langevin, Y., Matson, D. L., McCord, T. B., Mennella, V., Nelson, R. M., Nicholson, P. D., Sicardy, B., Sotin, C., Amici, S., Chamberlain, M. A., Filacchione, G., Hansen, G., Hibbitts, K., Showalter, M., 2003. Observations with the Visual and Infrared Mapping Spectrometer (VIMS) during Cassini's flyby of Jupiter. Icarus 164, 461-470. 
Böttger, H. M., Lewis, S. R., Read, P. L., Forget, F., 2005. The effects of the martian regolith on GCM water cycle simulations. Icarus 177, 174-189.

Carr, M. H. 1996. Water on Mars, Oxford University Press, New York

Chamberlain, M. A., Boynton, W. V., 2004. Modeling Depth to Ground Ice on Mars. Lunar and Planetary Institute Conference Abstracts 35, 1650.

Chamberlain, M. A., Boynton, W. V., 2005. Effect of Ground Ice on Apparent Thermal Inertia on Mars: Modeling Depth to Ground Ice on Mars. 36th Annual Lunar and Planetary Science Conference: Lunar and Planetary Institute Conference Abstracts 36, 1566.

Chamberlain, M. A., Brown, R. H., 2004. Near-infrared spectroscopy of Himalia. Icarus 172, 163-169.

Christensen, P. R., Bandfield, J. L., Hamilton, V. E., Ruff, S. W., Kieffer, H. H., Titus, T. N., Malin, M. C., Morris, R. V., Lane, M. D., Clark, R. L., Jakosky, B. M., Mellon, M. T., Pearl, J. C., Conrath, B. J., Smith, M. D., Clancy, R. T., Kuzmin, R. O., Roush, T. , Mehall, G. L., Gorelick, N., Bender, K., Murray, K., Dason, S., Greene, E., Silverman, S., Greenfield, M. 2001. Mars Global Surveyor Thermal Emission Spectrometer experiment: Investigation description and surface science results. Journal of Geophysical Research, 23823-23872.

Christensen, P. R., N.S. Gorelick, G.L. Mehall, and K.C. Murray THEMIS Public Data Releases. Planetary Data System node <http://themis-data.asu.edu>.

Clancy, R. T., Grossman, A. W., Wolff, M. J., James, P. B., Rudy, D. J., Billawala, Y. N., Sandor, B. J., Lee, S. W., Muhleman, D. O., 1996. Water vapor saturation at low altitudes around Mars aphelion: A key to Mars climate? Icarus 122, 36-62.

Clark, B. E., Helfenstein, P., Bell, J. F., Peterson, C., Veverka, J., Izenberg, N. I., Domingue, D., Wellnitz, D., McFadden, L., 2002. NEAR Infrared Spectrometer Photometry of Asteroid 433 Eros. Icarus 155, 189-204.

Clark, R. N., Swayze, G. A., Gallagher, A. J., King T. V. V., Clavin, W. M. 1993. US Geological Survey. digital spectral library: Version 1: 0.2 to 3.0 microns. US Geological Survey Open File Report 93-592.

Cruikshank, D. P., 1977. Radii and albedos of four Trojan asteroids and Jovian satellites 6 and 7. Icarus 30, 224-230.

Cruikshank, D. P., Dalle Ore, C. M., Roush, T. L., Geballe, T. R., Owen, T. C., de Bergh, C., Cash, M. D., Hartmann, W. K., 2001. Constraints on the Composition of Trojan Asteroid 624 Hektor. Icarus 153, 348-360. 
Davies, D. W., 1979. The relative humidity of Mars' atmosphere. J. Geophys. Res. 84, 8335-8340.

Davies, D. W., 1981. The Mars water cycle. Icarus 45, 398-414.

Degewij, J., Zellner, B., Andersson, L. E., 1980. Photometric properties of outer planetary satellites. Icarus 44, 520-540.

Dumas, C., Owen, T., Barucci, M. A., 1998. Near-Infrared Spectroscopy of Low-Albedo Surfaces of the Solar System: Search for the Spectral Signature of Dark Material. Icarus 133, 221-232.

Emery, J. P., Brown, R. H., 2003. Constraints on the surface composition of Trojan asteroids from near-infrared (0.8-4.0 mum) spectroscopy. Icarus 164, 104-121.

Fanale, F. P., Salvail, J. R., Zent, A. P., Postawko, S. E., 1986. Global distribution and migration of subsurface ice on Mars. Icarus 67, 1-18.

Feldman, W. C., Boynton, W. V., Tokar, R. L., Prettyman, T. H., Gasnault, O., Squyres, S. W., Elphic, R. C., Lawrence, D. J., Lawson, S. L., Maurice, S., McKinney, G. W., Moore, K. R., Reedy, R. C., 2002. Global Distribution of Neutrons from Mars: Results from Mars Odyssey. Science 297, 75-78.

Feldman, W. C., Mellon, M. T., Maurice, S., Prettyman, T. H., Carey, J. W., Vaniman, D. T., Bish, D. L., Fialips, C. I., Chipera, S. J., Kargel, J. S., Elphic, R. C., Funsten, H. O., Lawrence, D. J., Tokar, R. L., 2004a. Hydrated states of $\mathrm{MgSO}_{4}$ at equatorial latitudes on Mars. Geophys. Res. Lett. 31, 16702.

Feldman, W. C., Prettyman, T. H., Maurice, S., Plaut, J. J., Bish, D. L., Vaniman, D. T., Mellon, M. T., Metzger, A. E., Squyres, S. W., Karunatillake, S., Boynton, W. V., Elphic, R. C. , Funsten, H. O., Lawrence, D. J., Tokar, R. L. 2004b. Global distribution of near-surface hydrogen on Mars . J. Geophys. Res. 109.

Geballe, T. R., Dalle Ore, C. M., Cruikshank, D. P., Owen, T. C., 2002. The 1.95-2.50 mum Spectrum of J6 Himalia. Icarus 159, 542-544.

Gehrels, T., 1967. Minor planets. I. The rotation of Vesta. Astron. J. 72, 929.

Haberle, R. M., 2004. History and Progress of GCM Simulations on Recent Mars Climate Change. Lunar and Planetary Institute Conference Abstracts 35, 2010.

Haberle, R. M., Jakosky, B. M. 1991. Atmospheric effects on the remote determination of thermal inertia on Mars . Icarus 90, 187-204.

Haberle, R. M., Pollack, J. B., Barnes, J. R., Zurek, R. W., Leovy, C. B., Murphy, J. R., Lee, H., Schaeffer, J., 1993. Mars atmospheric dynamics as simulated by the 
NASA AMES General Circulation Model. I - The zonal-mean circulation. J. Geophys. Res. 98, 3093-3123.

Haberle, R. M., Murphy, J. R., Schaeffer, J., 2003. Orbital change experiments with a Mars general circulation model. Icarus 161, 66-89.

Hapke, B. 1993. Theory of reflectance and emittance spectroscopy, Cambridge University Press, Cambridge, UK.

Head, J. W., Marchant, D. R., 2003. Cold-based mountain glaciers on Mars: Western Arsia Mons. Geology 31[7], 641-644.

Helfenstein, P. and Veverka, J. 1989. Physical characterization of asteroid surfaces from photometric analysis. In: Binzel R. P., Gehrels T., Matthews M. S. (Eds.), Asteroids II. Univ. of Arizona Press, Tucson, pp. 557-593.

Hinnov, L. A., 2000. New Perspectives on Orbitally Forced Stratigraphy. Annual Review of Earth and Planetary Sciences 28, 419-475.

Houben, H., Haberle, R. M., Young, R. E., Zent, A. P., 1997. Modeling the Martian seasonal water cycle. J. Geophys. Res. 102, 9069-9084.

Jakosky, B. M., Carr, M. H., 1985. Possible precipitation of ice at low latitudes of Mars during periods of high obliquity. Nature 315, 559-561.

Jakosky, B. M., Farmer, C. B., 1982. The seasonal and global behavior of water vapor in the Mars atmosphere - Complete global results of the Viking atmospheric water detector experiment. J. Geophys. Res. 87, 2999-3019.

Jakosky, B. M., Henderson, B. G., Mellon, M. T. 1993. The Mars water cycle at other epochs - Recent history of the polar caps and layered terrain. Icarus 102, 286-297.

Jakosky, B. M., 1983. The role of seasonal reservoirs in the Mars water cycle II. Coupled models of the regolith, the polar caps, and atmospheric transport. Icarus 55, 1939.

Jakosky, B. M., Mellon, M. T., Varnes, E. S., Feldman, W. C., Boynton, W. V., Haberle, R. M., 2005. Mars low-latitude neutron distribution: Possible remnant nearsurface water ice and a mechanism for its recent emplacement. Icarus 175[1], 5867.

Jakosky, B. M., Zent, A. P., Zurek, R. W., 1997. The Mars Water Cycle: Determining the Role of Exchange with the Regolith. Icarus 130[1], 87-95.

James, P. B., 1985. The Martian hydrologic cycle - Effects of CO2 mass flux on global water distribution. Icarus 64, 249-264. 
James, P. B., Cantor, B. A., 2001. Martian North Polar Cap Recession: 2000 Mars Orbiter Camera Observations. Icarus 154, 131-144.

Jarvis, K. S., Vilas, F., Larson, S. M., Gaffey, M. J., 2000. JVI Himalia: New compositional evidence and interpretations for the origin of Jupiter's small satellites. Icarus 145, 445-453.

Kelly, N. J., Boynton, W. V., Kerry, K., Hamara, D., Janes, D., Reedy, R. C., Kim, K. J., Haberle, R. M., 2006. Seasonal Polar Carbon Dioxide Frost on Mars: CO2 Mass and Columnar Thickness Distribution. JGR in press.

Kieffer, H. H., Jakosky, B. M., and Snyder, C. W. 1992. The planet Mars - From antiquity to the present. In: Kieffer H. H., Jakosky B. M., Snyder C. W., Matthews M. S. (Eds.), Mars. Univ. of Arizona Press, Tucson, pp. 1-33.

Kieffer, H. H., Titus, T. N., 2001. TES Mapping of Mars' North Seasonal Cap. Icarus $154,162-180$.

Kieffer, H. H. and Zent, A. P. 1992. Quasi-periodic climate change on Mars. In: Kieffer H. H., Jakosky B. M., Snyder C. W., Matthews M. S. (Eds.), Mars. Univ. of Arizona Press, Tucson, pp. 1180-1218.

Laskar, J., Correia, A. C. M., Gastineau, M., Joutel, F., Levrard, B., Robutel, P. 2004. Long term evolution and chaotic diffusion of the insolation quantities of Mars . Icarus 170, 343-364.

Leighton, R. R., Murray, B. C. 1966. Behavior of carbon dioxide and other volatiles on Mars. Science 153, 136-144.

Leovy, C., Wood, S. E., Catling, D., Montgomery, D. R., Moore, J., Barnhart, C., Ginder, E., Louie, M., 2004. Evidence for Possible Exposed Water Ice Deposits in Martian Low Latitude Chasms and Chaos. Lunar and Planetary Institute Conference Abstracts 35, 2016.

Levrard, B., Forget, F., Montmessin, F., Laskar, J., 2004. Recent ice-rich deposits formed at high latitudes on Mars by sublimation of unstable equatorial ice during low obliquity. Nature 431, 1072-1075.

Lucchitta, B. K., 1981. Mars and earth - Comparison of cold-climate features. Icarus 45, 264-303.

Luu, J., 1991. CCD photometry and spectroscopy of the outer Jovian satellites. Astron. J. 102, 1213-1225.

Malin, M. C., Caplinger, M. A., Davis, S. D., 2001. Observational Evidence for an Active 
Surface Reservoir of Solid Carbon Dioxide on Mars. Science 294[5549], 21462148 .

McCord, T. B., Coradini, A., Hibbitts, C. A., Capaccioni, F., Hansen, G. B., Filacchione, G., Clark, R. N., Cerroni, P., Brown, R. H., Baines, K. H., Bellucci, G., Bibring, J.-P., Buratti, B. J., Bussoletti, E., Combes, M., Cruikshank, D. P., Drossart, P., Formisano, V., Jaumann, R., Langevin, Y., Matson, D. L., Nelson, R. M., Nicholson, P. D., Sicardy, B., Sotin, C., 2004. Cassini VIMS observations of the Galilean satellites including the VIMS calibration procedure. Icarus 172, 104-126.

Mellon, M. T., Jakosky, B. M. 1993. Geographic variations in the thermal and diffusive stability of ground ice on Mars. J. Geophys. Res. 98, 3345-3364 .

Mellon, M. T., Feldman, W. C., Prettyman, T. H. 2004. The presence and stability of ground ice in the southern hemisphere of Mars. Icarus 169, 324-340.

Mellon, M. T., Jakosky, B. M. 1995. The distribution and behavior of Martian ground ice during past and present epochs . J. Geophys. Res. 100, 11781-11799.

Milankovitch, M. M., 1941. Kanon der Erdbestrahlung und seine Anwendung auf das Eiszeitproblem. 133, 1-633.Koniglich Serbische Akademie, Belgrade.

Miller, E. A., Klein, G., Juergens, D. W., Mehaffey, K., Oseas, J. M., Garcia, R. A., Giandomenico, A., Irigoyen, R. E., Hickok, R., Rosing, D., Sobel, H. R., Bruce, C. F., Flamini, E., Devidi, R., Reininger, F. M., Dami, M., Soufflot, A., Langevin, Y., Huntzinger, G., 1996. The Visual and Infrared Mapping Spectrometer for Cassini. Proc. SPIE Vol. 2803, p. 206-220, Cassini/Huygens: A Mission to the Saturnian Systems, Linda Horn; Ed. 2803, 206-220.

Milliken, R. E., Mustard, J. F., 2003. Erosional Morphologies and Characteristics of Latitude-dependent Surface Mantles on Mars. Sixth International Conference on Mars , 3240.

Mischna, M. A., Richardson, M. I. 2005. A reanalysis of water abundances in the Martian atmosphere at high obliquity . Geophys. Res. Lett. 32.

Mischna, M. A., Richardson, M. I., Wilson, R. J. , McCleese, D. J. 2003 . On the orbital forcing of Martian water and $\mathrm{CO} 2$ cycles: A general circulation model study with simplified volatile schemes. J. Geophys. Res. 108, 16-1.

Mooney, T., Knacke, R. F., 1985. Optical constants of chlorite and serpentine between 2.5 and 50 microns. Icarus 64, 493-502.

Murray, B. C., Ward, W. R., Yeung W. C. 1973. Periodic insolation variations on Mars. Science 180, 638-640. 
Mustard, J. F., Cooper, C. D., Rifkin, M. K., 2001. Evidence for recent climate change on Mars from the identification of youthful near-surface ground ice. Nature 412, 411-414.

NASA, 2004. Mars rovers spot water-clue mineral, frost, clouds. NASA press release 2004-287.

Paige, D. A., Ingersoll, A. P., 1985. Annual heat balance of Martian polar caps - Viking observations. Science 228, 1160-1168.

Paige, D. A., 1992. The thermal stability of near-surface ground ice on Mars. Nature 356, 43-45.

Paige, D. A., Bachman, J. E., Keegan, K. D. 1994. Thermal and albedo mapping of the polar regions of Mars using Viking thermal mapper observations: 1. North polar region. J. Geophys. Res. 99, 25959-25991.

Pollack, J. B., Burns, J. A., Tauber, M. E., 1979a. Gas drag in primordial circumplanetary envelopes - A mechanism for satellite capture. Icarus 37, 587-611.

Pollack, J. B., Colburn, D. S., Flasar, F. M., Kahn, R., Carlston, C. E., Pidek, D. G., 1979b. Properties and effects of dust particles suspended in the Martian atmosphere. J. Geophys. Res. 84, 2929-2945.

Pollack, J. B., Leovy, C. B., Greiman, P. W., Mintz, Y. 1981. A Martian general circulation experiment with large topography. Journal of the Atmospheric Sciences 38, 3-29.

Putzig, N. E., Mellon, M. T., Kretke, K. A., Arvidson, R. E., 2005. Global thermal inertia and surface properties of Mars from the MGS mapping mission. Icarus 173, 325341.

Reininger, F. M., Dami, M., Paolinetti, R., Pieri, S., Falugiani, S., 1994. Visible Infrared Mapping Spectrometer--visible channel (VIMS-V). Proc. SPIE Vol. 2198, p. 239250, Instrumentation in Astronomy VIII, David L. Crawford; Eric R. Craine; Eds. 2198, 239-250.

Rettig, T. W., Walsh, K., Consolmagno, G., 2001. Implied Evolutionary Differences of the Jovian Irregular Satellites from a BVR Color Survey. Icarus 154, 313-320.

Richardson, M. I., Wilson, R. J., 2002a. A topographically forced asymmetry in the martian circulation and climate. Nature 416, 298-301.

Richardson, M. I., Wilson, R. J., 2002b. Investigation of the nature and stability of the Martian seasonal water cycle with a general circulation model. J. Geophys. Res. 
107e, 7-1.

Ryan, J. A., Sharman, R. D., 1981. H2O frost point detection on Mars. J. Geophys. Res. $86,503-511$.

Ryan, J. A., Sharman, R. D., Lucich, R. D., 1982. Mars water vapor, near-surface. J. Geophys. Res. 87, 7279-7284.

Schorghofer, N., Aharonson, O. 2005. Stability and exchange of subsurface ice on Mars. J. Geophys. Res. 110, E05003, (10.1029/2004JE002350).

Smith, D., G. Neumann, R. E. Arvidson, E. A. Guinness, S. Slavney 2003. Mars Global Surveyor Laser Altimeter Mission Experiment Gridded Data Record. NASA Planetary Data System MGS-M-MOLA-5-MEGDR-L3-V1.0.

Smith, D. W., Johnson, P. E., Shorthill, R. W., 1981. Spectrophotometry of J8, J9, and four Trojan asteroids from 0.32 to 1.05 microns. Icarus $46,108-113$.

Smith, M. D. 2002. Annual cycle of water vapor on Mars as observed by the Thermal Emission Spectrometer. J. Geophys. Res. 107, 25-1.

Smith, M. D., 2004. Interannual variability in TES atmospheric observations of Mars during 1999-2003. Icarus 167, 148-165.

Spinrad, H., Münch, G., Kaplan, L. D. 1963. The Detection of Water Vapor on Mars. Astrophys. J. 137, 1319-1321.

Squyres, S. W., Carr, M. H., 1986. Geomorphic evidence for the distribution of ground ice on Mars. Science 231, 249-252.

Svitek, T., Murray, B. 1990. Winter frost at Viking Lander 2 site. J. Geophys. Res. 95, 1495-1510.

Sykes, M. V., Nelson, B., Cutri, R. M., Kirkpatrick, D. J., Hurt, R., Skrutskie, M. F., 2000. Near-Infrared Observations of the Outer Jovian Satellites. Icarus 143, 371375.

Taylor, R. C., Gehrels, T., Silvester, A. B. , 1971. Minor Planets and Related Objects. VI. Asteroid (110) Lydia. Astron. J. 76, 141.

Tedesco, E. F., Sather, R. E., 1981. Minor planets and related objects. XXIX - Asteroid 29 Amphitrite. Astron. J. 86, 1553-1558.

Tholen, D. J., Zellner, B., 1984. Multicolor photometry of outer Jovian satellites. Icarus $58,246-253$. 
Vilas, F., 1994. A cheaper, faster, better way to detect water of hydration on Solar System bodies. Icarus 111, 456-467.

Ward, W. R. 1992. Long-term orbital and spin dynamics of Mars. In: Kieffer H. H., Jakosky B. M., Snyder C. W., Matthews M. S. (Eds.), Mars. Tucson, Univ. of Arizona Press, pp. 298-320.

Warren, S. G., 1984. Optical constants of ice from the ultraviolet to the microwave. Appl. Opt. 23, 1206-1225.

Zappala, V., van Houten-Groeneveld, I., van Houten, C. J., 1979. Rotation period and phase curve of the asteroids 349 Dembowska and 354 Eleonora. Astronomy and Astrophysics Supplement Series 35, 213-221.

Zent, A. P., Haberle, R. M., Houben, H. C., Jakosky, B. M. 1993. A coupled subsurfaceboundary layer model of water on Mars. J. Geophys. Res. 98, 3319-3337.

Zent, A. P., Quinn, R. C. 1995. Simutaneous adsorption of $\mathrm{CO}_{2}$ and $\mathrm{H}_{2} \mathrm{O}$ under Mars-like conditions and application to the evolution of the Martian climate . J. Geophys. Res. 100, 5341-5349. 